Subvariedades bi-harmônicas de
variedades homogêneas tridimensionais

Apoenã Passos Passamani 


\title{
Subvariedades bi-harmônicas de variedades homogêneas tridimensionais
}

\author{
Apoenã Passos Passamani
}

Orientadora: Profa. Dra. Irene Ignazia Onnis

Dissertação apresentada ao Instituto de Ciências Matemáticas e de Computação - ICMC-USP, como parte dos requisitos para obtenção do título de Mestre em Ciências - Matemática. VERSÃO REVISADA.

USP - São Carlos

Junho/2011 
Ficha catalográfica elaborada pela Biblioteca Prof. Achille Bassi e Seção Técnica de Informática, ICMC/USP, com os dados fornecidos pelo(a) autor(a)

\begin{tabular}{|c|c|}
\hline \multirow[t]{3}{*}{ P $285 \mathrm{~s}$} & $\begin{array}{l}\text { Passamani, Apoenã Passos } \\
\quad \text { Subvariedades bi-harmônicas de variedades } \\
\text { homogêneas tridimensionais / Apoenã Passos } \\
\text { Passamani; orientadora Irene Ignazia Onnis -- São } \\
\text { Carlos, } 2011 \text {. } \\
\quad 73 \text { p. }\end{array}$ \\
\hline & $\begin{array}{l}\text { Dissertação (Mestrado - Programa de Pós-Graduação en } \\
\text { Matemática)-- Instituto de Ciências Matemáticas e } \\
\text { de Computação, Universidade de São Paulo, } 2011 .\end{array}$ \\
\hline & $\begin{array}{l}\text { 1. subvariedades bi-harmônicas. I. Onnis, Irene } \\
\text { Ignazia, orient. II. Título. }\end{array}$ \\
\hline
\end{tabular}


Às minhas três lindas e amáveis irmãs. 


\section{Agradecimentos}

A Deus, pelo seu cuidado e dedicação e por ter me dado força para concluir essa etapa da minha vida.

À minha amada família que sempre me apoiou e me deu muitas felicidades e orgulho. Em especial um agradecimento ao meu pai e a minha mãe, que estiveram ao meu lado, mesmo distante, dando força e incentivo nos momentos mais difíceis e por ter me estruturado para buscar e concluir mais este sonho.

À minha namorada que se mostrou uma verdadeira companheira, mesmo com a distância que nos separa.

Aos amigos, os de longa data que são quase irmãos e que nem a distância enfraqueceu nossa amizade. Aos recentes, que são queridos e que foram de vital importância para que eu cumprisse essa etapa de minha vida, longe de minha família.

Ao prof. Ricardo Soares Leite por me ajudar a reencontrar motivação em meus estudos e por ter sido um bom conselheiro.

À minha orientadora prof $^{a}$. Irene Ignazia Onnis por ter sido atenciosa, dedicada, incentivadora e extremamente paciente.

Por fim, agradeço a CNPq e a Fapesp pelo suporte financeiro. 


\section{Resumo}

Neste trabalho estudamos alguns resultados importantes sobre a teoria das subvariedades biharmônicas de espaços homogêneos tridimensionais. Existem três classes de espaços homogêneos tridimensionais simplesmente conexos dependendo da dimensão do grupo de isometrias, que pode ser: 3, 4 ou 6 . No caso da dimensão ser 6, $M$ é uma forma espacial; se a dimensão do grupo de isometrias for 4, $M$ é isométrica a: $\mathbb{H}_{3}$ (grupo de Heisenberg), $S U(2)$ (grupo unitário especial), $\widetilde{S L}(2, \mathbb{R})$ (revestimento universal do grupo linear especial), ou aos espaços produtos $\mathbb{S}^{2} \times \mathbb{R}$ e $\mathbb{H}^{2} \times \mathbb{R}$.

Feita exceção para $\mathbb{H}^{3}$, no caso da dimensão ser 4 ou 6 o espaço homogêneo é localmente isométrico a (uma parte de) $\mathbb{R}^{3}$, munido de uma métrica que depende de dois parâmetros reais. Tal família de métricas aparece primeiramente no trabalho [3] de L. Bianchi e, mais tarde, nos artigos $[14,35]$ de É. Cartan e G. Vranceanu, respectivamente.

Nesse projeto de mestrado, queremos estudar (essencialmente) resultados de existência e classificação de subvariedades bi-harmônicas nesses espaços, também conhecidos como variedades de Bianchi-Cartan-Vranceanu. 


\begin{abstract}
In this work we study some important results about the theory of the bi-harmonic submanifolds of 3-dimensional homogeneous spaces. There exist three classes of simply connected 3-dimensional homogeneous spaces depending on the dimension of the group of isometries, which can be: 3,4 or 6 . In the case of dimension $6, M$ will be a space form; if the dimension of the group of isometries is $4, M$ will be isometric to: either $\mathbb{H}_{3}$ (Heisenberg's group), or $S U(2)$ (special unitary group), or $\widetilde{S L}(2, \mathbb{R})$ (universal recovering of the special linear group), or the product spaces $\mathbb{S}^{2} \times \mathbb{R}$ and $\mathbb{H}^{2} \times \mathbb{R}$.

Except for $\mathbb{H}^{3}$, in the case of dimension 4 or 6 the homogeneous space is locally isometric to (a part of) $\mathbb{R}^{3}$, endowed with a metric that depends on two real parameters. Such family of metrics first appears in the work [3] of L. Bianchi and later in the articles [14, 35] of É. Cartan and G. Vranceanu, respectively.

In this master thesis, we want to study (essentially) results of existence and classification of bi-harmonic submanifolds in these spaces, also known as Bianchi-Cartan-Vranceanu's manifolds.
\end{abstract}




\section{Conteúdo}

Introdução 1

1 Bi-harmonicidade em geometria Riemanniana 5

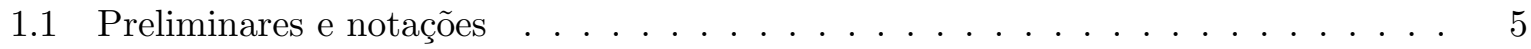

1.1.1 O operador de Beltrami-Laplace em variedades Riemannianas . . . . . . . 6

1.2 Definição de aplicação harmônica . . . . . . . . . . . . . . . . . . . . . . . 7

1.3 Aplicações bi-harmônicas . . . . . . . . . . . . . . . . . . . . . . . 12

1.3.1 A fórmula da primeira variação . . . . . . . . . . . . . . . . . . . 12

1.4 Condição de bi-harmonicidade para curvas . . . . . . . . . . . . . . . 16

1.4.1 A equação bi-harmônica em coordenadas locais . . . . . . . . . . . . . . . 17

2 Estudo das curvas bi-harmônicas com o método de Euler-Lagrange 19

2.1 Equivalência dos dois métodos f . . . . . . . . . . . . . . 20

3 Curvas bi-harmônicas próprias em uma variedade Riemanniana 31

3.1 Estudo das curvas bi-harmônicas em uma superfície . . . . . . . . . . . . . 33

3.2 Curvas bi-harmônicas em superfícies de revolução de $\mathbb{R}^{3} \ldots \ldots$. . . . . . . . 33

3.2.1 Exemplos de curvas bi-harmônicas em uma superfície de revolução com curvatura Gaussiana não constante . . . . . . . . . . . . . . . . . 36

3.2.2 Curvas bi-harmônicas em superfícies de revolução com curvatura Gaussiana constante ......................... 40

4 Classificação das curvas bi-harmônicas dos BCV-espaços 43

4.1 Espaços de Bianchi-Cartan-Vranceanu e sua estrutura Riemanniana . . . . . . . 43

4.2 Condições para curvas bi-harmônicas em $\left(M, g_{\ell, m}\right) \ldots \ldots \ldots \ldots \ldots$

4.3 Equações explícitas das curvas bi-harmônicas em $\left(M, g_{\ell, m}\right)$ com $\ell^{2} \neq 4 m$ e $m \neq 0 \quad 47$ 
4.4 Equações explícitas das curvas bi-harmônicas próprias em $\mathbb{H}_{3} \ldots \ldots \ldots$. . . . . . 54

4.5 Curvas bi-harmônicas próprias da esfera $\mathbb{S}^{3} \ldots \ldots \ldots \ldots \ldots$. . . . . . . 57

5 Superfícies bi-harmônicas em forma espaciais $\quad 63$

5.1 Resultados preliminares na esfera $\mathbb{S}^{n} \ldots \ldots \ldots \ldots \ldots \ldots$

5.2 Superfícies bi-harmônicas da esfera $\mathbb{S}^{3} \ldots \ldots \ldots \ldots \ldots \ldots$ 


\section{Introdução}

Indicaremos por $C^{\infty}(M, N)$ o espaço das aplicações diferenciáveis entre duas variedades Riemannianas $M$ e $N$. Uma função $f \in C^{\infty}(M, N)$, com $M$ compacta, é dita harmônica se é um ponto crítico do funcional energia:

$$
E: C^{\infty}(M, N) \rightarrow \mathbb{R}, \quad E(f)=\frac{1}{2} \int_{M}\|d f\|^{2} d M
$$

ou seja ([22]), se $f$ é uma solução da correspondente equação de Euler-Lagrange dada por

$$
\tau(f)=\operatorname{tr} \widetilde{\nabla} d f=0
$$

onde $\tau(f)$ é o campo de tensão de $f$ e $\widetilde{\nabla}$ denota a conexão Riemanniana do fibrado $T^{*} M \otimes f^{-1} T N$.

A equação (1) é conhecida como equacão harmônica e, em coordenadas locais $\left\{x_{i}\right\}$ em $M$ e $\left\{y_{\alpha}\right\}$ em $N$, assume a forma:

$$
\tau(f)=\left(-\triangle^{M} f_{\alpha}+g^{i j N} \Gamma_{\beta \delta}^{\alpha} \frac{\partial f_{\beta}}{\partial x_{i}} \frac{\partial f_{\delta}}{\partial x_{j}}\right) \frac{\partial}{\partial y_{\alpha}} \circ f=0,
$$

onde ${ }^{N} \Gamma_{\beta \delta}^{\alpha}$ são os símbolos de Christoffel de $(N, h)$ e $\triangle^{M}$ o operador de Beltrami-Laplace em $(M, g)$.

Exemplo 0.0.1. Seja $N=\mathbb{R}^{n}$ munido da métrica canônica e $f: M \rightarrow \mathbb{R}^{n}$ uma aplicação diferenciável, $f=\left(f_{1}, \ldots, f_{n}\right)$. Como $\Gamma_{\beta \delta}^{\alpha}=0$, então $f$ é harmonica se, e somente se, $\triangle^{M} f_{\alpha}=0$, $\alpha=1, \ldots, n$.

Exemplo 0.0.2 (Geodésicas). Uma aplicação $f:(a, b) \rightarrow\left(N^{n}, h\right)$ é harmônica se, e somente, se

$$
\frac{d^{2} f_{\alpha}}{d t^{2}}+\Gamma_{\beta \delta}^{\alpha} \frac{d f_{\beta}}{d t} \frac{d f_{\delta}}{d t}=0, \quad \alpha=1, \ldots, n,
$$

ou seja, se e somente se $f$ é uma geodésica. 
As aplicações bi-harmônicas generalizam o conceito de aplicação harmônica (como sugerido por J. Eells e J.H. Sampson em [22]) e são definidas como pontos críticos do funcional bi-energia:

$$
E_{2}: C^{\infty}(M, N) \rightarrow \mathbb{R}, \quad E_{2}(f)=\frac{1}{2} \int_{M}\|\tau(f)\|^{2} d M
$$

Em [26, 27] G.Y. Jiang obteve a fórmula da primeira variação da bi-energia provando que a equação de Euler-Lagrange para $E_{2}$ é dada por

$$
\tau_{2}(f)=-\Delta \tau(f)+\operatorname{tr} R^{N}(d f, \tau(f)) d f=0
$$

onde, denotando por $\bar{\nabla}$ a conexão no fibrado $f^{-1} T N, \Delta=-\operatorname{tr}\left(\bar{\nabla} \bar{\nabla}-\bar{\nabla}_{\nabla}\right)$ é o Laplaciano definido nas seções de $f^{-1} T N$ e $R^{N}(X, Y)=\nabla_{Y}^{\prime} \nabla_{X}^{\prime}-\nabla_{X}^{\prime} \nabla_{Y}^{\prime}+\nabla_{[X, Y]}^{\prime}$ é o operador de curvatura em $T N$, definido através da conexão desse fibrado $\nabla^{\prime}$. A equação (2) é chamada de equação bi-harmônica.

Da expressão do campo de bi-tensão $\tau_{2}$ segue que toda aplicação harmônica (i.e. $\tau=0$ ) é também bi-harmônica. Uma aplicação bi-harmônica que não é harmônica é chamada de aplicação bi-harmônica própria.

O estudo das funções bi-harmônicas teve inicio em 1863 devido a sua ligação com a teoria da elasticidade e com a mecânica dos fluidos; G.B. Airy e J.C. Maxwell foram os primeiros a usar tal tipo de funções para descrever um modelo matemático de elasticidade (veja [1, 29]). Já a teoria das funções poli-harmônicas se desenvolveu mais tarde por obra de E. Almansi, T. LeviCivita e M. Nicolaescu. Recentemente, R. Caddeo e L. Vanhecke ([13]), L. Sario, M. Nakai, C. Wang e L. Chung (veja [33]), entre outros, começaram o estudo das funções harmônicas e poli-harmônicas em variedades Riemannianas.

Na última década o estudo das aplicações e das imersões bi-harmônicas tem gerado nos pesquisadores um interesse crescente como demonstra a lista das publicações sobre o assunto presente no site "http://people.unica.it/biharmonic/", de autoria de E. Loubeau, S. Montaldo e C. Oniciuc.

Neste trabalho são apresentados alguns resultados importantes sobre a teoria das subvariedades bi-harmônicas (ou seja, das subvariedades tais que a inclusão é uma aplicação bi-harmônica) de espaços homogêneos tridimensionais.

Existem três classes de espaços homogêneos tridimensionais simplesmente conexos dependendo da dimensão do grupo de isometrias, que pode ser: 3,4 ou 6 . No caso da dimensão ser 
6, $M$ é uma forma espacial; se a dimensão do grupo de isometrias for $4, M$ é isométrica a: $\mathbb{H}_{3}$ (grupo de Heisenberg tridimensional), $S U(2)$ (grupo unitário especial), $\widetilde{S L}(2, \mathbb{R}$ ) (revestimento universal do grupo linear especial), ou aos espaços produtos $\mathbb{S}^{2} \times \mathbb{R}$ ou $\mathbb{H}^{2} \times \mathbb{R}$.

Feita exceção para o espaço hiperbólico tridimensional $\mathbb{H}^{3}$, no caso da dimensão ser 4 ou 6 o espaço homogêneo é localmente isométrico a (uma parte de) $\mathbb{R}^{3}$, munido de uma métrica que depende de dois parâmetros reais. Tal família de métricas aparece primeiramente no trabalho [3] de L. Bianchi e, mais tarde, nos artigos [14, 35] de É. Cartan e G. Vranceanu, respectivamente.

Este trabalho se encaixa nesta linha, com ênfase no estudo de resultados de existência e classificação de subvariedades bi-harmônicas nas variedades de Bianchi-Cartan-Vranceanu (ou BCV-espaços).

No Capítulo 1 damos a definição de aplicações bi-harmônicas entre variedades Riemannianas como generalização natural do conceito de aplicação harmônica. Também, provaremos a fórmula da primeira variação da energia (dada em [31]) e da bi-energia (dada em [27]).

No Capítulo 2 apresentamos o método de Euler-Lagrange, que faz uso de uma bi-lagrangiana, para o estudo de curvas bi-harmônicas e que resulta em um sistema de equações diferenciais ordinárias. Em seguida é demonstrada a equivalência entre a equação bi-harmônica e o sistema de equações diferenciais ordinárias obtido no presente capítulo, assim como foi feito por R. Caddeo, S. Montaldo, C. Oniciuc e P. Piu em [10]. Por fim aplicamos o método de Euler-Lagrange para determinar as curvas bi-harmônicas do plano hiperbólico e do grupo de Lie Sol.

O Capítulo 3 é resultado do estudo do artigo [11], onde é feita a discussão sobre as curvas bi-harmônicas em superfícies. Começamos considerando o caso geral, de uma superfície qualquer, depois nos restringimos às superfícies de revolução do espaço Euclidiano tridimensional. São, então, descritas curvas bi-harmônicas de algumas superfícies como o toro de revolução, as superfícies de Delaunay e o elipsóide de revolução e, também, damos a solução explícita da equação bi-harmônica para o caso de superfícies de revolução com curvatura Gaussiana constante positiva.

Já no Capítulo 4 damos a classificação completa de curvas bi-harmônicas nas 3-variedades de Bianchi-Cartan-Vranceanu $\left(M, g_{\ell, m}\right)$, que são definidas como sendo (uma parte de) $\mathbb{R}^{3}$ com uma métrica dependente de dois parâmetros reais $\ell, m$. No início apresentamos tais espaços e sua estrutura Riemanniana, e obtemos condições para as curvas bi-harmônicas nestes espaços. Depois fazemos um estudo específico, começando com o caso $\ell^{2} \neq 4 m$ e $m \neq 0$ (ou seja, os 
espaços $\mathbb{S}^{2} \times \mathbb{R}, \mathbb{H}^{2} \times \mathbb{R}, S U(2)$ e $\left.\widetilde{S L}(2, \mathbb{R})\right)$ seguindo o que fizeram R. Caddeo, S. Montaldo, C. Oniciuc e P. Piu em [9]. Depois analisamos o caso $\ell=1$ e $m=0$ (i.e. o espaço de Heisenberg $\mathbb{H}_{3}$ ) que foi estudado em [12]. Por fim, consideramos o caso de $\mathbb{S}^{3}$ (i.e. $\ell^{2}=4 m \neq 0$ ) estudado em [8]. Em cada um desses casos expomos as equações explícitas das curvas bi-harmônicas.

O Capítulo 5 finaliza este texto e é dedicado ao estudo de superfícies bi-harmônicas próprias das formas espaciais tridimensionais (veja [8]). A inexistência de tais superfícies em formas espaciais com curvatura seccional $c \leq 0$, nos leva a nos restringirmos ao caso da esfera $\mathbb{S}^{3}$, para qual demonstramos que as superfícies bi-harmônicas próprias são hiperesferas de raio $1 / \sqrt{2}$. 


\section{Capítulo 1}

\section{Bi-harmonicidade em geometria}

\section{Riemanniana}

O presente capítulo tem como fim introduzir o conceito de aplicação bi-harmônica entre duas variedades Riemannianas, de forma tal que o mesmo apareça como generalização natural da noção de aplicação harmônica. Primeiramente definiremos aplicações harmônicas $f:(M, g) \rightarrow(N, h)$ entre variedades Riemannianas como ponto crítico do funcional energia, provando (veja Teorema 1.2.3) que a correspondente equação de Euler-Lagrange é $\tau(f)=0$ (onde $\tau(f)$ denota o campo de tensão de $f$ ). Em seguida, veremos que a aplicação $f$ é dita bi-harmônica se é ponto crítico do funcional bi-energia. Neste caso a correspondente equação de Euler-Lagrange foi descrita por G.Y. Jiang em [27] e é dada por $\tau_{2}(f)=0$ (onde $\tau_{2}(f)$ denota o campo de bi-tensão de $f$ ).

\subsection{Preliminares e notações}

Seja $f:(M, g) \rightarrow(N, h)$ uma aplicação $C^{\infty}$. Usaremos os símbolos $\nabla, \nabla^{\prime}, \bar{\nabla}$ e $\widetilde{\nabla}$ para as conexões Riemannianas nos fibrados $T M, T N, f^{-1} T N=\bigcup_{p \in M} T_{f(p)} N$ e $T^{*} M \otimes f^{-1} T N$, respectivamente. De forma que, por definição (ver, por exemplo, [34] e [21]), temos:

$$
\bar{\nabla}_{X} V=\nabla_{d f(X)}^{\prime} V \quad \text { e } \quad(\widetilde{\nabla} d f)(X, Y)=\left(\widetilde{\nabla}_{X} d f\right)(Y)=\bar{\nabla}_{X} d f(Y)-d f\left(\nabla_{X} Y\right)
$$

onde $X, Y \in \Gamma(T M)$ e

$$
V \in \Gamma\left(f^{-1} T N\right)=\left\{W \mid W: M \rightarrow T N \text { é uma aplicação } C^{\infty}, W(p) \in T_{f(p)} N, p \in M\right\} .
$$


Além disso, o tensor curvatura $\widetilde{\mathrm{R}}$ no fibrado $T^{*} M \otimes f^{-1} T N$ é definindo como segue

$$
\widetilde{\mathrm{R}}(X, Y)=\widetilde{\nabla}_{Y} \widetilde{\nabla}_{X}-\widetilde{\nabla}_{X} \widetilde{\nabla}_{Y}+\widetilde{\nabla}_{[X, Y]}, \quad X, Y \in \Gamma(T M) .
$$

Portanto, para qualquer $Z \in \Gamma(T M)$, definimos

$$
\begin{aligned}
(\widetilde{\mathrm{R}}(X, Y) d f)(Z) & =\mathrm{R}^{f^{-1} T N}(X, Y) d f(Z)-d f\left(\mathrm{R}^{M}(X, Y) Z\right) \\
& =\mathrm{R}^{N}(d f(X), d f(Y)) d f(Z)-d f\left(\mathrm{R}^{M}(X, Y) Z\right),
\end{aligned}
$$

onde $\mathrm{R}^{M}, \mathrm{R}^{N}$ e $\mathrm{R}^{f^{-1} T N}$ são os tensores de curvatura em $T M, T N$ e $f^{-1} T N$, respectivamente.

\subsubsection{O operador de Beltrami-Laplace em variedades Riemannianas}

Consideremos $(M, g)$ uma variedade Riemanniana de dimensão $m$ e $\left(U,\left\{x_{i}\right\}\right)$ um sistema de coordenadas locais. Denotamos por $g=\operatorname{det}\left(g_{i k}\right)$, sendo

$$
\left(g_{i k}\right)=\left(g\left(\frac{\partial}{\partial x_{i}}, \frac{\partial}{\partial x_{k}}\right)\right)
$$

a matriz associada à métrica $g$.

Definição 1.1.1. Seja $f \in C^{\infty}(M)$ e $X=X_{i} \frac{\partial}{\partial x_{i}} \in \Gamma(T M)$, definimos o gradiente de $f$ sobre $(M, g)$ e a divergência de $X$ como

$$
\operatorname{grad} f=\sum_{i, k} g^{i k}\left(\frac{\partial f}{\partial x_{i}}\right) \frac{\partial}{\partial x_{k}}
$$

$e$

$$
\operatorname{div} X=\frac{1}{\sqrt{g}} \sum_{i} \frac{\partial}{\partial x_{i}}\left(X_{i} \sqrt{g}\right)
$$

respectivamente.

Observação 1.1.2. Se considerarmos $\left\{e_{k}\right\}$ uma base local de campos ortonormais, uma expressão equivalente para a divergência (ver, por exemplo, [34]) é dada por

$$
\operatorname{div} X=\sum_{k}\left\langle\nabla_{e_{k}} X, e_{k}\right\rangle
$$

Por meio desses operadores, introduzimos na variedade $(M, g)$ o operador de BeltramiLaplace $\triangle^{M}: C^{\infty}(M) \rightarrow C^{\infty}(M)$ por

$$
\triangle^{M} f=\operatorname{div}(\operatorname{grad} f),
$$


que, em coordenadas locais, tem expressão

$$
\triangle^{M} f=\frac{1}{\sqrt{g}} \sum_{i, j} \frac{\partial}{\partial x_{i}}\left(g^{i j} \sqrt{g} \frac{\partial f}{\partial x_{j}}\right),
$$

de onde se obtém (ver [34])

$$
\begin{aligned}
\triangle^{M} f & =\sum_{i, j, k} g^{i j}\left(\frac{\partial^{2} f}{\partial x_{i} \partial x_{j}}-\Gamma_{i j}^{k} \frac{\partial f}{\partial x_{k}}\right) \\
& =\sum_{i}\left\{e_{i}\left(e_{i}(f)\right)-\left(\nabla_{e_{i}} e_{i}\right) f\right\}
\end{aligned}
$$

Observação 1.1.3. Observe que se $\left\{e_{i}\right\}$ for um referencial (local) geodésico em $p \in M$, então

$$
\begin{aligned}
\operatorname{grad} f(p) & =\sum_{i}\left(e_{i}(f)\right) e_{i}(p), \\
\operatorname{div} X(p) & =\sum_{i} e_{i}\left(f_{i}\right)(p), \quad X=\sum_{i} f_{i} e_{i}, \\
\triangle^{M} f(p) & =\sum_{i} e_{i}\left(e_{i}(f)\right)(p) .
\end{aligned}
$$

Exemplo 1.1.4. Se $M=\mathbb{R}^{m}$ com sua estrutura Riemanniana usual, então

$$
\triangle^{M} f=\sum_{i} \frac{\partial^{2} f}{\partial x_{i}^{2}}, \quad f \in C^{\infty}(M) .
$$

\subsection{Definição de aplicação harmônica}

Seja $C^{\infty}(M, N)$ o espaço das aplicações diferenciáveis $f:(M, g) \rightarrow(N, h)$ entre as duas variedades Riemannianas $M$ e $N$. Se $f \in C^{\infty}(M, N)$, podemos ver a diferencial $d f$ como uma seção do fibrado $T^{*} M \otimes f^{-1} T N$ e então sua norma é dada por

$$
\begin{aligned}
\|d f\|_{p}^{2} & =\langle d f, d f\rangle_{p}=\sum_{i}\left\langle d f_{p}\left(e_{i}\right), d f_{p}\left(e_{i}\right)\right\rangle_{f(p)} \\
& =g^{i j}(p) f_{\alpha}^{i}(p) f_{\beta}^{j}(p) h_{\alpha \beta}(f(p)),
\end{aligned}
$$

onde $\left\{e_{i}\right\}_{i=1, \ldots, m}$ é uma base ortonormal de $T_{p} M$. A norma $\|d f\|_{p}$ é conhecida como norma de Hilbert-Schmidt da aplicação linear $d f_{p}$.

Definição 1.2.1. A densidade de energia de uma aplicação $f \in C^{\infty}(M, N)$ é a função $e(f)=$ $\frac{1}{2}\|d f\|^{2}$, e a energia de $f$ é dada por $E(f)=\int_{M} e(f) d M$. 
Definição 1.2.2. Uma aplicação $f \in C^{\infty}(M, N)$ é dita harmônica se é um ponto crítico do funcional energia $E: C^{\infty}(M, N) \rightarrow \mathbb{R}$ ou seja, se para toda variação suave de $f,\left\{f_{t}\right\}$, com $-\epsilon<t<\epsilon$, temos

$$
\left.\frac{d}{d t}\right|_{t=0} E\left(f_{t}\right)=0
$$

Neste contesto, ao dizermos variação suave $\left\{f_{t}\right\}$, queremos dizer que $f_{0}=f$ e que a dependência de $\left\{f_{t}\right\}$ com respeito a $t$ é de classe $C^{\infty}$; ou seja, a aplicação

$$
F:(-\epsilon, \epsilon) \times M \rightarrow N
$$

definida por

$$
F(t, p):=f_{t}(p), \quad-\epsilon<t<\epsilon, p \in M,
$$

satisfaz

$$
\left\{\begin{array}{l}
F(0, p)=f(p), \quad p \in M, \\
F:(-\epsilon, \epsilon) \times M \rightarrow N \text { é uma aplicação } C^{\infty} .
\end{array}\right.
$$

Para cada variação suave $\left\{f_{t}\right\}$ de $f,-\epsilon<t<\epsilon$, pondo

$$
V(p):=\left.\frac{d}{d t}\right|_{t=0} f_{t}(p), \quad p \in M
$$

então $V$ é uma aplicação $C^{\infty}$ de $M$ no fibrado tangente $T N$ satisfazendo

$$
V(p) \in T_{f(p)} N, \quad p \in M
$$

Reciprocamente, para cada aplicação $C^{\infty}, V: M \rightarrow T N$, satisfazendo (1.14), definindo

$$
f_{t}(p):=\exp _{f(p)}(t V(p)), \quad p \in M
$$

resulta que $f_{t} \in C^{\infty}(M, N)$ e

$$
\left.\frac{d}{d t}\right|_{t=0} f_{t}(p)=V(p), \quad p \in M
$$

Tal campo de vetores $V$ é chamado de campo de vetores variacional ao longo de $f^{1}$.

\footnotetext{
${ }^{1}$ Campos de vetores variacionais podem ser vistos como seções de algum fibrado sobre $M$, da maneira que passamos a explicar.

Seja $f^{-1} T N$ o fibrado sobre $M$, induzido por $f$ a partir de $T N$ e $\pi: T N \rightarrow N$ a projeção canônica. Então o conjunto de todas as seções $C^{\infty}$ de $f^{-1} T N$, denotado por $\Gamma\left(f^{-1} T N\right)$, é o conjunto de todos os campos de vetores variacionais:

$$
\Gamma\left(f^{-1} T N\right)=\left\{V \mid V: M \rightarrow T N \text { é uma aplicação } C^{\infty} \text { e } V(p) \in T_{f(p)} N, p \in M\right\}
$$
}


Considere $F: I_{\epsilon} \times M \rightarrow N, I_{\epsilon}=(-\epsilon, \epsilon)$, a função induzida pela variação $\left\{f_{t}\right\}$, como em (1.12). Adotando a métrica Euclidiana usual em $I_{\epsilon}$, com respeito à métrica Riemanniana produto em $I_{\epsilon} \times M$, denotaremos por $\boldsymbol{\nabla}, \bar{\nabla}, \widetilde{\nabla}$, as conexões Riemannianas induzidas em $T\left(I_{\epsilon} \times M\right)$, $F^{-1} T N, T^{*}\left(I_{\epsilon} \times M\right) \otimes F^{-1} T N$, respectivamente. Se $\left\{e_{i}\right\}$ for uma base ortonormal de campos definidos em uma vizinhança $U$ de $p,\left\{\frac{\partial}{\partial t}, e_{i}\right\}$ será também uma base ortonormal de campos em uma vizinhança coordenada em $I_{\epsilon} \times U$, e vale

$$
\nabla_{\frac{\partial}{\partial t}} \frac{\partial}{\partial t}=0, \quad \nabla_{e_{i}} e_{j}=\nabla_{e_{i}} e_{j}, \quad \nabla_{\frac{\partial}{\partial t}} e_{i}=\nabla_{e_{i}} \frac{\partial}{\partial t}=0
$$

Além disso, temos que

$$
\frac{\partial f_{t}}{\partial t}=\frac{\partial F_{\alpha}}{\partial t} \frac{\partial}{\partial y_{\alpha}}=d F\left(\frac{\partial}{\partial t}\right), \quad d f_{t}\left(e_{i}\right)=d F\left(e_{i}\right)
$$

e

$$
\begin{aligned}
& \left(\widetilde{\nabla}_{e_{i}} d f_{t}\right)\left(e_{j}\right)=\nabla_{d f_{t}\left(e_{i}\right)}^{\prime} d f_{t}\left(e_{j}\right)-d f_{t}\left(\nabla_{e_{i}} e_{j}\right)=\left(\widetilde{\nabla}_{e_{i}} d F\right)\left(e_{j}\right), \\
& \left(\widetilde{\nabla}_{e_{k}} \widetilde{\nabla}_{e_{i}} d f_{t}\right)\left(e_{j}\right)=\nabla_{d f_{t}\left(e_{k}\right)}^{\prime}\left(\left(\widetilde{\nabla}_{e_{i}} d f_{t}\right)\left(e_{j}\right)\right)-\left(\widetilde{\nabla}_{e_{i}} d f_{t}\right)\left(\nabla_{e_{k}} e_{j}\right)=\left(\widetilde{\nabla}_{e_{k}} \widetilde{\nabla}_{e_{i}} d F\right)\left(e_{j}\right),
\end{aligned}
$$

etc. Aqui usamos abreviadamente o símbolo $\widetilde{\nabla}$ em $T^{*} M \otimes f_{t}^{-1} T N$ no qual omitimos $t$.

Vale o seguinte resultado:

Teorema 1.2.3 ([22]). Uma aplicação $f:(M, g) \rightarrow(N, h)$ é harmônica se e somente se

$$
\tau(f)=\operatorname{tr} \widetilde{\nabla} d f=0
$$

Demonstração. Seja $\left\{f_{t}\right\}$ uma variação suave de $f$, definimos $F: I_{\epsilon} \times M \rightarrow N$ pondo $F(t, p)=$ $f_{t}(p)$. Denotamos por $V$ o campo variacional correspondente. A energia de $f_{t}$ é dada por $E\left(f_{t}\right)=\int_{M} e\left(f_{t}\right) d M$, e então

$$
\left.\frac{d}{d t}\right|_{t=0} E\left(f_{t}\right)=\left.\frac{d}{d t}\right|_{t=0} \int_{M} e\left(f_{t}\right) d M=\left.\int_{M} \frac{d}{d t}\right|_{t=0} e\left(f_{t}\right) d M .
$$

Seja $p \in M$ e considere um sistema de coordenadas normais $\left(U,\left\{x_{i}\right\}\right)$ em $M$ e seus correspondentes campos tangentes $\left\{e_{i}\right\}_{i=1, \ldots, m}$ em $p$, de forma que $\left\langle e_{i}, e_{j}\right\rangle=\delta_{i j}$ e $\left(\nabla_{e_{i}} e_{i}\right)(p)=0$. Como

$$
\begin{aligned}
\left.\frac{d}{d t}\right|_{t} e\left(f_{t}\right)(p) & =\left.\frac{1}{2} \frac{d}{d t}\right|_{t}\left\{\sum_{i}\left\langle d f_{t}\left(e_{i}\right), d f_{t}\left(e_{i}\right)\right\rangle_{p}\right\} \\
& =\frac{1}{2}\left(\frac{\partial}{\partial t}\right)_{(t, p)}\left\{\sum_{i}\left\langle d F\left(e_{i}\right), d F\left(e_{i}\right)\right\rangle\right\} \\
& =\sum_{i}\left\langle\bar{\nabla}_{\frac{\partial}{\partial t}} d F\left(e_{i}\right), d F\left(e_{i}\right)\right\rangle_{(t, p)}
\end{aligned}
$$


e vale

$$
\bar{\nabla}_{X}(d F(Y))-\bar{\nabla}_{Y}(d F(X))-d F([X, Y])=0,
$$

para todo $X, Y \in \Gamma\left(T\left(I_{\epsilon} \times M\right)\right)$ (ver [34], Lema 1.16), temos

$$
\left.\frac{d}{d t}\right|_{t} e\left(f_{t}\right)(p)=\sum_{i}\left\langle\bar{\nabla}_{e_{i}} d F\left(\frac{\partial}{\partial t}\right)+d F\left(\left[\frac{\partial}{\partial t}, e_{i}\right]\right), d F\left(e_{i}\right)\right\rangle_{(t, p)} .
$$

Portanto,

$$
\begin{aligned}
\left.\frac{d}{d t}\right|_{t=0} e\left(f_{t}\right)(p) & =\sum_{i}\left\langle\bar{\nabla}_{e_{i}} V, d f\left(e_{i}\right)\right\rangle_{p} \\
& \left.=\sum_{i}\left\{e_{i}\left\langle V, d f\left(e_{i}\right)\right)\right\rangle_{p}-\left\langle V, \bar{\nabla}_{e_{i}} d f\left(e_{i}\right)\right\rangle_{p}\right\}
\end{aligned}
$$

Seja $q \in M$ e $\left\{Y_{i}\right\}_{i=1, \ldots, m}$ uma base ortonormal de $T_{q} M$. Definimos o campo de vetores

$$
X(q)=\sum_{i}\left\langle V, d f\left(Y_{i}\right)\right\rangle_{q} Y_{i} \in T_{q} M
$$

Tal campo está bem definido, no sentido de que não depende da escolha da base ortonormal $\left\{Y_{i}\right\}$. Ao variar $q$ em $M$, obtemos $X \in \Gamma(T M)$ e, então, pela fórmula (1.8), temos que

$$
(\operatorname{div} X)(p)=\sum_{i=1} e_{i}\left\langle V, d f\left(e_{i}\right)\right\rangle(p)
$$

Substituindo (1.21) em (1.20) obtemos

$$
\begin{aligned}
\left.\frac{d}{d t}\right|_{t=0} e\left(f_{t}\right)(p) & =(\operatorname{div} X)(p)-\left\langle V, \sum_{i} \widetilde{\nabla} d f\left(e_{i}, e_{i}\right)\right\rangle_{p} \\
& =(\operatorname{div} X)(p)-\langle V, \tau(f)\rangle_{p} .
\end{aligned}
$$

Da integração de (1.22), resulta

$$
\left.\frac{d}{d t}\right|_{t=0} E\left(f_{t}\right)=-\int_{M}\langle V, \tau(f)\rangle d M
$$

O resultado segue devido à arbitrariedade de $V$.

A equação $\tau(f)=0$ é dita equação harmônica. Vamos agora escrever $\tau(f)$ em coordenadas locais $\left\{x_{i}\right\}$ em $M$ e $\left\{y_{\alpha}\right\}$ em $N$. Temos que:

$$
\tau(f)=\operatorname{tr} \widetilde{\nabla} d f=g^{i j}(\widetilde{\nabla} d f)\left(\frac{\partial}{\partial x_{i}}, \frac{\partial}{\partial x_{j}}\right)
$$


onde

$$
\begin{aligned}
(\widetilde{\nabla} d f)\left(\frac{\partial}{\partial x_{i}}, \frac{\partial}{\partial x_{j}}\right) & =\left(\widetilde{\nabla}_{\frac{\partial}{\partial x_{i}}} d f\right)\left(\frac{\partial}{\partial x_{j}}\right) \\
& =\bar{\nabla}_{\frac{\partial}{\partial x_{i}}} d f\left(\frac{\partial}{\partial x_{j}}\right)-d f\left(\nabla_{\frac{\partial}{\partial x_{i}}} \frac{\partial}{\partial x_{j}}\right) \\
& =\bar{\nabla}_{\frac{\partial}{\partial x_{i}}}\left(\frac{\partial f_{\alpha}}{\partial x_{j}}\left(\frac{\partial}{\partial y_{\alpha}} \circ f\right)\right)-\Gamma_{i j}^{k} \frac{\partial f_{\alpha}}{\partial x_{k}}\left(\frac{\partial}{\partial y_{\alpha}} \circ f\right) \\
& =\frac{\partial^{2} f_{\alpha}}{\partial x_{i} \partial x_{j}}\left(\frac{\partial}{\partial y_{\alpha}} \circ f\right)+\frac{\partial f_{\alpha}}{\partial x_{j}} \frac{\partial f_{\beta}}{\partial x_{i}}\left(\nabla_{\frac{\partial}{\partial y_{\beta}}}^{\prime} \frac{\partial}{\partial y_{\alpha}} \circ f\right)-\Gamma_{i j}^{k} \frac{\partial f_{\alpha}}{\partial x_{k}}\left(\frac{\partial}{\partial y_{\alpha}} \circ f\right) \\
& =\left(\frac{\partial^{2} f_{\alpha}}{\partial x_{i} \partial x_{j}}-\Gamma_{i j}^{k} \frac{\partial f_{\alpha}}{\partial x_{k}}+{ }^{N} \Gamma_{\beta \sigma}^{\alpha} \frac{\partial f_{\sigma}}{\partial x_{j}} \frac{\partial f_{\beta}}{\partial x_{i}}\right)\left(\frac{\partial}{\partial y_{\alpha}} \circ f\right) .
\end{aligned}
$$

Portanto, usando (1.7), resulta

$$
\tau(f)=\left(\triangle^{M} f_{\alpha}+g^{i j N} \Gamma_{\beta \sigma}^{\alpha} \frac{\partial f_{\sigma}}{\partial x_{j}} \frac{\partial f_{\beta}}{\partial x_{i}}\right)\left(\frac{\partial}{\partial y_{\alpha}} \circ f\right)
$$

onde $\triangle^{M}$ é o operador de Beltrami-Laplace em $(M, g)$.

Exemplo 1.2.4. Seja $f:(M, g) \rightarrow(N, h)$ uma aplicação constante, ou seja, $f(p)=q$ para todo $p \in M$. Então $\tau(f)=0$.

Exemplo 1.2.5 (Geodésicas). Uma curva $f:(-\epsilon, \epsilon) \rightarrow(N, h)$ é uma aplicação harmônica se e somente se é geodésica, ou seja

$$
\frac{d^{2} f_{\alpha}}{d t^{2}}+\Gamma_{\beta \delta}^{\alpha} \frac{d f_{\beta}}{d t} \frac{d f_{\delta}}{d t}=0, \quad \alpha=1, \ldots, n
$$

Exemplo 1.2.6 (Imersões mínimas). Seja $f:(M, g) \rightarrow(N, h)$ uma imersão isométrica, i.e., $f^{*} h=g$. Identificando $X \in \Gamma(T M) \operatorname{com} d f(X) \in \Gamma\left(f^{-1} T N\right)$, vemos que a segunda forma fundamental II pode ser escrita como

$$
\mathrm{II}(X, Y)=\nabla_{X}^{\prime} Y-\nabla_{X} Y=\widetilde{\nabla} d f(X, Y) .
$$

Então, pela definição do vetor curvatura média $\mathrm{H}$ de $f$, temos

$$
\tau(f)=\operatorname{tr} \widetilde{\nabla} d f=m \mathrm{H}
$$

Portanto, $f$ é harmônica se e somente se é minimal.

Exemplo 1.2.7. A aplicação identidade $1:(M, g) \rightarrow(M, g)$ é harmônica. 


\subsection{Aplicações bi-harmônicas}

Em [22] J. Eells e J. H. Sampson generalizaram o conceito de aplicações harmônicas introduzindo a noção de aplicações $k$-harmônicas. Nesta seção apresentaremos os principais resultados dados por G.Y. Jiang em [27] provando a fórmula da primeira variação das aplicações bi-harmônicas (i.e. $k=2$ ).

Nos cálculos que seguem omitiremos o símbolo de somatório para que a notação fique mais leve.

Na Seção 1.2 definimos o funcional energia e escrevemos sua correspondente equação de EulerLagrange chegando, dessa forma, à condição de harmonicidade para uma aplicação $f \in C^{\infty}(M, N)$. Para esta aplicação, definimos o funcional bi-energia, pondo

$$
E_{2}(f)=\frac{1}{2} \int_{M}\|\tau(f)\|^{2} d M
$$

onde $\tau(f)$ é o campo de tensão da aplicação $f$. Podemos agora dar a seguinte

Definição 1.3.1. Seja $f:(M, g) \rightarrow(N, h)$ uma aplicação $C^{\infty}$, entre variedades Riemannianas quaisquer. Dizemos que f é uma aplicação bi-harmônica se é um ponto crítico do funcional bienergia em $C^{\infty}(M, N)$, i.e., se para toda variação suave de $f,\left\{f_{t}\right\} \in C^{\infty}(M, N)$ com $-\epsilon<t<\epsilon$, temos

$$
\left.\frac{d}{d t}\right|_{t=0} E_{2}\left(f_{t}\right)=0
$$

\subsubsection{A fórmula da primeira variação}

No que segue, provaremos dois lemas que serão úteis no cálculo da primeira variação de $E_{2}$, para isso usaremos as notações dadas na Seção 1.2.

Lema 1.3.2 ([27]). Para qualquer variação $C^{\infty},\left\{f_{t}\right\}$ de $f$, vale

$$
\begin{aligned}
\frac{d}{d t} E_{2}\left(f_{t}\right) & =\int_{M}\left\langle\left(\widetilde{\boldsymbol{\nabla}}_{e_{i}} \widetilde{\boldsymbol{\nabla}}_{e_{i}} d F\right)\left(\frac{\partial}{\partial t}\right)-\left(\widetilde{\boldsymbol{\nabla}}_{\nabla_{e_{i}} e_{i}} d F\right)\left(\frac{\partial}{\partial t}\right),\left(\widetilde{\nabla}_{e_{j}} d F\right)\left(e_{j}\right)\right\rangle d M \\
& +\int_{M}\left\langle\mathrm{R}^{N}\left(d F\left(e_{i}\right), d F\left(\frac{\partial}{\partial t}\right)\right) d F\left(e_{i}\right),\left(\widetilde{\boldsymbol{\nabla}}_{e_{j}} d F\right)\left(e_{j}\right)\right\rangle d M
\end{aligned}
$$

Demonstração. Sendo $\left\{e_{i}\right\}$ uma base ortonormal local, a equação (1.26) pode ser reescrita como

$$
2 E_{2}(f)=\int_{M}\|\tau(f)\|^{2} d M=\int_{M}\left\langle\left(\widetilde{\nabla}_{e_{i}} d f\right)\left(e_{i}\right),\left(\widetilde{\nabla}_{e_{j}} d f\right)\left(e_{j}\right)\right\rangle d M .
$$


Devido a (1.17), para uma variação $\left\{f_{t}\right\}$ de $f$, vale que

$$
\begin{aligned}
2 \frac{d}{d t} E_{2}\left(f_{t}\right) & =\frac{d}{d t} \int_{M}\left\langle\left(\widetilde{\nabla}_{e_{i}} d F\right)\left(e_{i}\right),\left(\widetilde{\nabla}_{e_{j}} d F\right)\left(e_{j}\right)\right\rangle d M \\
& =2 \int_{M}\left\langle\overline{\boldsymbol{\nabla}}_{\frac{\partial}{\partial t}}\left(\left(\widetilde{\nabla}_{e_{i}} d F\right)\left(e_{i}\right)\right),\left(\widetilde{\nabla}_{e_{j}} d F\right)\left(e_{j}\right)\right\rangle d M .
\end{aligned}
$$

De (1.15) e usando o tensor curvatura em $T^{*}\left(I_{\epsilon} \times M\right) \otimes F^{-1} T N$, temos

$$
\begin{aligned}
\left(\widetilde{\mathrm{R}}\left(X, \frac{\partial}{\partial t}\right) d F\right)(Y) & =\mathrm{R}^{N}\left(d F(X), d F\left(\frac{\partial}{\partial t}\right)\right) d F(Y) \\
& -d F\left(\mathrm{R}^{M \times I_{\epsilon}}\left(X, \frac{\partial}{\partial t}\right) Y\right) \\
& =\mathrm{R}^{N}\left(d F(X), d F\left(\frac{\partial}{\partial t}\right)\right) d F(Y),
\end{aligned}
$$

para todo $X, Y \in \Gamma\left(T\left(I_{\epsilon} \times M\right)\right)$. Além disso, como

$$
\bar{\nabla}_{X}(d F(Y))-\bar{\nabla}_{Y}(d F(X))-d F([X, Y])=0,
$$

para todo $X, Y \in \Gamma\left(T\left(I_{\epsilon} \times M\right)\right)$, temos

$$
\begin{aligned}
\left(\widetilde{\boldsymbol{\nabla}}_{e_{i}} d F\right)\left(\frac{\partial}{\partial t}\right) & =\bar{\nabla}_{e_{i}}\left(d F\left(\frac{\partial}{\partial t}\right)\right)-d F\left(\boldsymbol{\nabla}_{e_{1}} \frac{\partial}{\partial t}\right) \\
& =\bar{\nabla}_{e_{i}}\left(d F\left(\frac{\partial}{\partial t}\right)\right) \\
& =\bar{\nabla}_{\frac{\partial}{\partial t}}\left(d F\left(e_{i}\right)\right)+d F\left(\left[e_{i}, \frac{\partial}{\partial t}\right]\right) \\
& =\bar{\nabla}_{\frac{\partial}{\partial t}}\left(d F\left(e_{i}\right)\right) \\
& =\bar{\nabla}_{\frac{\partial}{\partial t}}\left(d F\left(e_{i}\right)\right)-d F\left(\boldsymbol{\nabla}_{\frac{\partial}{\partial t}} e_{i}\right) \\
& =\left(\widetilde{\nabla}_{\frac{\partial}{\partial t}} d F\right)\left(e_{i}\right)
\end{aligned}
$$

e, de forma análoga,

$$
\left(\widetilde{\nabla}_{\frac{\partial}{\partial t}} d F\right)\left(\nabla_{e_{i}} e_{i}\right)=\left(\widetilde{\nabla}_{\nabla_{e_{i}} e_{i}} d F\right)\left(\frac{\partial}{\partial t}\right) .
$$

Agora podemos trocar a ordem das diferenciações em $\bar{\nabla}_{\frac{\partial}{\partial t}}\left(\left(\widetilde{\nabla}_{e_{i}} d F\right)\left(e_{i}\right)\right)$ :

$$
\begin{aligned}
\overline{\boldsymbol{\nabla}}_{\frac{\partial}{\partial t}}\left(\left(\widetilde{\boldsymbol{\nabla}}_{e_{i}} d F\right)\left(e_{i}\right)\right) & =\widetilde{\boldsymbol{\nabla}}_{\frac{\partial}{\partial t}}\left(\left(\widetilde{\boldsymbol{\nabla}}_{e_{i}} d F\right)\left(e_{i}\right)\right)+\left(\widetilde{\boldsymbol{\nabla}}_{e_{i}} d F\right)\left(\boldsymbol{\nabla}_{\frac{\partial}{\partial t}} e_{i}\right) \\
& =\left(\widetilde{\boldsymbol{\nabla}}_{\frac{\partial}{\partial t}} \widetilde{\boldsymbol{\nabla}}_{e_{i}} d F\right)\left(e_{i}\right) \\
& =\left(\widetilde{\boldsymbol{\nabla}}_{e_{i}} \widetilde{\boldsymbol{\nabla}}_{\frac{\partial}{\partial t}} d F-\widetilde{\boldsymbol{\nabla}}_{\left[e_{i}, \frac{\partial}{\partial t}\right]} d F+\widetilde{\mathrm{R}}\left(e_{i}, \frac{\partial}{\partial t}\right) d F\right)\left(e_{i}\right) \\
& =\overline{\boldsymbol{\nabla}}_{e_{i}}\left(\left(\widetilde{\boldsymbol{\nabla}}_{\frac{\partial}{\partial t}} d F\right)\left(e_{i}\right)\right)-\left(\widetilde{\boldsymbol{\nabla}}_{\frac{\partial}{\partial t}} d F\right)\left(\nabla_{e_{i}} e_{i}\right) \\
& +\mathrm{R}^{N}\left(d F\left(e_{i}\right), d F\left(\frac{\partial}{\partial t}\right)\right) d F\left(e_{i}\right) \\
& =\bar{\nabla}_{e_{i}}\left(\left(\widetilde{\boldsymbol{\nabla}}_{e_{i}} d F\right)\left(\frac{\partial}{\partial t}\right)\right)-\left(\widetilde{\nabla}_{\nabla_{e_{i}} e_{i}} d F\right)\left(\frac{\partial}{\partial t}\right) \\
& +\mathrm{R}^{N}\left(d F\left(e_{i}\right), d F\left(\frac{\partial}{\partial t}\right)\right) d F\left(e_{i}\right) \\
& =\left(\widetilde{\boldsymbol{\nabla}}_{e_{i}} \widetilde{\boldsymbol{\nabla}}_{e_{i}} d F\right)\left(\frac{\partial}{\partial t}\right)-\left(\widetilde{\nabla}_{\nabla_{e_{i}} e_{i}} d F\right)\left(\frac{\partial}{\partial t}\right) \\
& +\mathrm{R}^{N}\left(d F\left(e_{i}\right), d F\left(\frac{\partial}{\partial t}\right)\right) d F\left(e_{i}\right) .
\end{aligned}
$$


O resultado segue ao substituirmos (1.32) em (1.30).

Lema 1.3.3 ([27]). Seguindo a notação acima e supondo que $M$ seja compacta, vale

$$
\begin{gathered}
\int_{M}\left\langle\left(\widetilde{\boldsymbol{\nabla}}_{e_{i}} \widetilde{\boldsymbol{\nabla}}_{e_{i}} d F\right)\left(\frac{\partial}{\partial t}\right)-\left(\widetilde{\boldsymbol{\nabla}}_{\nabla_{e_{i}} e_{i}} d F\right)\left(\frac{\partial}{\partial t}\right),\left(\widetilde{\boldsymbol{\nabla}}_{e_{j}} d F\right)\left(e_{j}\right)\right\rangle d M \\
=\int_{M}\left\langle d F\left(\frac{\partial}{\partial t}\right), \bar{\nabla}_{e_{k}} \overline{\boldsymbol{\nabla}}_{e_{k}}\left(\left(\widetilde{\boldsymbol{\nabla}}_{e_{j}} d F\right)\left(e_{j}\right)\right)-\overline{\boldsymbol{\nabla}}_{\nabla_{e_{k}} e_{k}}\left(\left(\widetilde{\boldsymbol{\nabla}}_{e_{j}} d F\right)\left(e_{j}\right)\right)\right\rangle d M .
\end{gathered}
$$

Demonstração. Para cada $t \in I_{\epsilon}$, definimos um campo de vetores $C^{\infty}$ sobre $M$, pondo

$$
X=\left\langle\left(\widetilde{\nabla}_{e_{i}} d F\right)\left(\frac{\partial}{\partial t}\right),\left(\widetilde{\nabla}_{e_{j}} d F\right)\left(e_{j}\right)\right\rangle e_{i}
$$

o qual está bem definido devido a sua independência com respeito à escolha da base $\left\{e_{i}\right\}$. De (1.5), resulta que

$$
\begin{aligned}
\operatorname{div} X & =\left\langle\nabla_{e_{k}} X, e_{k}\right\rangle_{M}=e_{i}\left(\left\langle\left(\widetilde{\nabla}_{e_{i}} d F\right)\left(\frac{\partial}{\partial t}\right),\left(\widetilde{\nabla}_{e_{j}} d F\right)\left(e_{j}\right)\right\rangle\right) \\
& +\left\langle\left(\widetilde{\nabla}_{e_{i}} d F\right)\left(\frac{\partial}{\partial t}\right),\left(\widetilde{\nabla}_{e_{j}} d F\right)\left(e_{j}\right)\right\rangle\left\langle\nabla_{e_{k}} e_{i}, e_{k}\right\rangle_{M} .
\end{aligned}
$$

Utilizando (1.15) e observando que

$$
\left\langle\nabla_{e_{k}} e_{i}, e_{k}\right\rangle_{M}+\left\langle e_{i}, \nabla_{e_{k}} e_{k}\right\rangle_{M}=0
$$

obtemos:

$$
\begin{aligned}
\operatorname{div} X & =\left\langle\overline{\boldsymbol{\nabla}}_{e_{i}}\left(\widetilde{\boldsymbol{\nabla}}_{e_{i}} d F\right)\left(\frac{\partial}{\partial t}\right),\left(\widetilde{\boldsymbol{\nabla}}_{e_{j}} d F\right)\left(e_{j}\right)\right\rangle+\left\langle\left(\widetilde{\boldsymbol{\nabla}}_{e_{i}} d F\right)\left(\frac{\partial}{\partial t}\right), \overline{\boldsymbol{\nabla}}_{e_{i}}\left(\left(\widetilde{\boldsymbol{\nabla}}_{e_{j}} d F\right)\left(e_{j}\right)\right)\right\rangle \\
& -\left\langle\left(\widetilde{\boldsymbol{\nabla}}_{e_{i}} d F\right)\left(\frac{\partial}{\partial t}\right),\left(\widetilde{\boldsymbol{\nabla}}_{e_{j}} d F\right)\left(e_{j}\right)\right\rangle\left\langle e_{i}, \nabla_{e_{k}} e_{k}\right\rangle_{M} \\
& =\left\langle\left(\widetilde{\boldsymbol{\nabla}}_{e_{i}} \widetilde{\boldsymbol{\nabla}}_{e_{i}} d F\right)\left(\frac{\partial}{\partial t}\right)+\left(\widetilde{\boldsymbol{\nabla}}_{e_{i}} d F\right)\left(\boldsymbol{\nabla}_{e_{i}} \frac{\partial}{\partial t}\right),\left(\widetilde{\boldsymbol{\nabla}}_{e_{j}} d F\right)\left(e_{j}\right)\right\rangle \\
& +\left\langle\left(\widetilde{\boldsymbol{\nabla}}_{e_{i}} d F\right)\left(\frac{\partial}{\partial t}\right), \overline{\boldsymbol{\nabla}}_{e_{i}}\left(\left(\widetilde{\boldsymbol{\nabla}}_{e_{j}} d F\right)\left(e_{j}\right)\right)\right\rangle-\left\langle\left(\widetilde{\boldsymbol{\nabla}}_{\left\langle e_{i}, \nabla_{e_{k}} e_{k}\right\rangle_{M} e_{i}} d F\right)\left(\frac{\partial}{\partial t}\right),\left(\widetilde{\boldsymbol{\nabla}}_{e_{j}} d F\right)\left(e_{j}\right)\right\rangle \\
& =\left\langle\left(\widetilde{\boldsymbol{\nabla}}_{e_{i}} \widetilde{\boldsymbol{\nabla}}_{e_{i}} d F\right)\left(\frac{\partial}{\partial t}\right),\left(\widetilde{\boldsymbol{\nabla}}_{e_{j}} d F\right)\left(e_{j}\right)\right\rangle+\left\langle\left(\widetilde{\boldsymbol{\nabla}}_{e_{i}} d F\right)\left(\frac{\partial}{\partial t}\right), \overline{\boldsymbol{\nabla}}_{e_{i}}\left(\left(\widetilde{\boldsymbol{\nabla}}_{e_{j}} d F\right)\left(e_{j}\right)\right)\right\rangle \\
& -\left\langle\left(\widetilde{\boldsymbol{\nabla}}_{\nabla_{e_{k}} e_{k}} d F\right)\left(\frac{\partial}{\partial t}\right),\left(\widetilde{\boldsymbol{\nabla}}_{e_{j}} d F\right)\left(e_{j}\right)\right\rangle .
\end{aligned}
$$

Também definimos um campo de vetores $C^{\infty}$ sobre $M$ pondo

$$
Y=\left\langle d F\left(\frac{\partial}{\partial t}\right), \bar{\nabla}_{e_{i}}\left(\left(\widetilde{\nabla}_{e_{j}} d F\right)\left(e_{j}\right)\right)\right\rangle e_{i}
$$


Observe que $Y$ está bem definido e que

$$
\begin{aligned}
\operatorname{div} Y & =\left\langle\nabla_{e_{k}} Y, e_{k}\right\rangle_{M} \\
& =\left\langle\left(\widetilde{\nabla}_{e_{k}} d F\right)\left(\frac{\partial}{\partial t}\right), \bar{\nabla}_{e_{k}}\left(\left(\widetilde{\nabla}_{e_{j}} d F\right)\left(e_{j}\right)\right)\right\rangle+\left\langle d F\left(\frac{\partial}{\partial t}\right), \bar{\nabla}_{e_{k}} \bar{\nabla}_{e_{k}}\left(\left(\widetilde{\nabla}_{e_{j}} d F\right)\left(e_{j}\right)\right)\right\rangle \\
& -\left\langle d F\left(\frac{\partial}{\partial t}\right), \bar{\nabla}_{\nabla_{e_{k}} e_{k}}\left(\left(\widetilde{\nabla}_{e_{j}} d F\right)\left(e_{j}\right)\right)\right\rangle .
\end{aligned}
$$

Pelo Teorema de Green, temos

$$
\int_{M} \operatorname{div}(X-Y) d M=0
$$

e então, juntando (1.37) e (1.39) à equação (1.40), chegamos à equação (1.33).

Agora estamos prontos para determinar a primeira variação de $E_{2}$.

Teorema 1.3.4. Seja $f: M \rightarrow N$ uma aplicação $C^{\infty}$ de uma variedade Riemanniana compacta $M$, em uma variedade Riemanniana qualquer $N$. Seja $\left\{f_{t}\right\}$ uma variação $C^{\infty}$ que gera o campo de vetores $V$. Então,

$$
\left.\frac{d}{d t}\right|_{t=0} E_{2}\left(f_{t}\right)=\int_{M}\left\langle V, \bar{\nabla}_{e_{i}} \bar{\nabla}_{e_{i}} \tau(f)-\bar{\nabla}_{\nabla_{e_{i}} e_{i}} \tau(f)+\mathrm{R}^{N}\left(d f\left(e_{i}\right), \tau(f)\right) d f\left(e_{i}\right)\right\rangle d M
$$

Demonstração. Substituindo (1.33) em (1.28), temos a expressão

$$
\begin{aligned}
\frac{d}{d t} E_{2}\left(f_{t}\right) & =\int_{M}\left\langle d F\left(\frac{\partial}{\partial t}\right), \bar{\nabla}_{e_{k}} \bar{\nabla}_{e_{k}}\left(\left(\widetilde{\nabla}_{e_{j}} d F\right)\left(e_{j}\right)\right)-\bar{\nabla}_{\nabla_{e_{k}} e_{k}}\left(\left(\widetilde{\nabla}_{e_{j}} d F\right)\left(e_{j}\right)\right)\right\rangle d M \\
& +\int_{M}\left\langle\mathrm{R}^{N}\left(d F\left(e_{i}\right), d F\left(\frac{\partial}{\partial t}\right)\right) d F\left(e_{i}\right),\left(\widetilde{\boldsymbol{\nabla}}_{e_{j}} d F\right)\left(e_{j}\right)\right\rangle d M
\end{aligned}
$$

Fazendo a substituição

$$
\begin{aligned}
& \left\langle\mathrm{R}^{N}\left(d F\left(e_{i}\right), d F\left(\frac{\partial}{\partial t}\right)\right) d F\left(e_{i}\right),\left(\widetilde{\nabla}_{e_{j}} d F\right)\left(e_{j}\right)\right\rangle \\
& =\left\langle\mathrm{R}^{N}\left(d F\left(e_{i}\right),\left(\widetilde{\nabla}_{e_{j}} d F\right)\left(e_{j}\right)\right) d F\left(e_{i}\right), d F\left(\frac{\partial}{\partial t}\right)\right\rangle,
\end{aligned}
$$

pondo $t=0$ e utilizando (1.13), (1.16) e (1.17), obtemos a equação desejada.

Observação 1.3.5. Nos argumentos acima foi utilizada a hipótese de que $M$ é uma variedade Riemanniana compacta sem bordo. Para uma variedade Riemanniana qualquer $M$, seja $D \subset M$ um domínio limitado arbitrário com fronteira suave, e tome uma variação $\left\{f_{t}\right\}$ de $f$ satisfazendo

$$
\left.\frac{\partial f_{t}}{\partial t}\right|_{\partial D}=0,\left.\quad\left(\bar{\nabla}_{e_{i}} \frac{\partial f_{t}}{\partial t}\right)\right|_{\partial D}=0
$$


Então, no Lema 1.3.3, obtemos (1.33) ao aplicarmos o Teorema da divergência de Green a $X$ e $Y$. Logo, temos a fórmula da primeira variação em $D$ como sendo

$$
\left.\frac{d}{d t}\right|_{t=0} E_{2}\left(f_{t}, D\right)=\int_{D}\left\langle V, \bar{\nabla}_{e_{i}} \bar{\nabla}_{e_{i}} \tau(f)-\bar{\nabla}_{\nabla_{e_{i}} e_{i}} \tau(f)+\mathrm{R}^{N}\left(d f\left(e_{i}\right), \tau(f)\right) d f\left(e_{i}\right)\right\rangle d M,
$$

onde $E_{2}\left(f_{t}, D\right)$ é o correspondente funcional relativo a $D$.

Observação 1.3.6. Cabe aqui ressaltar que, devido a arbitrariedade do campo $V$, a condição analítica que obtemos para aplicações bi-harmonicas é

$$
\bar{\nabla}_{e_{i}} \bar{\nabla}_{e_{i}} \tau(f)-\bar{\nabla}_{\nabla_{e_{i}} e_{i}} \tau(f)+\mathrm{R}^{N}\left(d f\left(e_{i}\right), \tau(f)\right) d f\left(e_{i}\right)=0
$$

Então, de forma natural, damos a definição que segue.

Definição 1.3.7. Seja $f \in C^{\infty}(M, N)$. Chamamos de campo de bi-tensão de $f$ o campo

$$
\tau_{2}(f)=-\Delta_{f}(\tau(f))+\mathrm{R}_{f}(\tau(f))
$$

onde

$$
\Delta_{f}(\tau(f))=-\left(\bar{\nabla}_{e_{i}} \bar{\nabla}_{e_{i}}-\bar{\nabla}_{\nabla_{e_{i}} e_{i}}\right)(\tau(f))
$$

é o Laplaciano generalizado, enquanto

$$
\mathrm{R}_{f}(\tau(f))=\mathrm{R}^{N}\left(d f\left(e_{i}\right), \tau(f)\right) d f\left(e_{i}\right) .
$$

Com estas notações, a equação bi-harmônica (1.44) se torna

$$
\tau_{2}(f)=0 .
$$

Da expressão de $\tau_{2}$ segue que toda aplicação harmônica (i.e. $\tau=0$ ) é também bi-harmônica. Uma aplicação bi-harmônica que não é harmônica é dita aplicação bi-harmônica própria.

\subsection{Condição de bi-harmonicidade para curvas}

Considere uma imersão isométrica $\gamma: I \rightarrow(M, g)$ de um intervalo $I \subseteq \mathbb{R}$ em uma variedade Riemanniana $M$. Supondo que $\gamma$ esteja parametrizada pelo comprimento de arco, temos

$$
e_{1}=\frac{d}{d t} \quad \text { e } \quad d \gamma\left(\frac{d}{d t}\right)=\gamma^{\prime} .
$$

Como ${ }^{\mathbb{R}} \nabla_{\frac{d}{d t}} \frac{d}{d t}=0$, a equação bi-harmônica se torna

$$
\tau_{2}(\gamma)=\nabla_{\gamma^{\prime}}^{2} \tau(\gamma)+\mathrm{R}\left(\gamma^{\prime}, \tau(\gamma)\right) \gamma^{\prime}=0
$$




\subsubsection{A equação bi-harmônica em coordenadas locais}

Daremos à equação (1.46), a qual deve ser satisfeita pelas curvas bi-harmônicas de uma variedade Riemanniana $n$-dimensional $M$, uma representação em coordenadas locais. Sendo

$$
\begin{aligned}
\mathrm{R}\left(\gamma^{\prime}, \tau(\gamma)\right) \gamma^{\prime} & =\nabla_{\left[\gamma^{\prime}, \tau(\gamma)\right]} \gamma^{\prime}-\left[\nabla_{\gamma^{\prime}}, \nabla_{\tau(\gamma)}\right] \gamma^{\prime} \\
& =\nabla_{\left(\nabla_{\gamma^{\prime}} \tau(\gamma)-\nabla_{\tau(\gamma)} \gamma^{\prime}\right)} \gamma^{\prime}-\nabla_{\gamma^{\prime}} \nabla_{\tau(\gamma)} \gamma^{\prime}+\nabla_{\tau(\gamma)} \nabla_{\gamma^{\prime}} \gamma^{\prime} \\
& =\nabla_{\left(\nabla_{\gamma^{\prime}} \tau(\gamma)\right)} \gamma^{\prime}-\nabla_{\left(\nabla_{\tau(\gamma)} \gamma^{\prime}\right)} \gamma^{\prime}-\nabla_{\gamma^{\prime}} \nabla_{\tau(\gamma)} \gamma^{\prime}+\nabla_{\tau(\gamma)} \nabla_{\gamma^{\prime}} \gamma^{\prime}
\end{aligned}
$$

substituindo em (1.46) resulta:

$$
\begin{aligned}
\tau_{2}(\gamma) & =\sum_{k=1}^{n}\left(\nabla_{\gamma^{\prime}}^{2} \tau(\gamma)+\mathrm{R}\left(\gamma^{\prime}, \tau(\gamma)\right) \gamma^{\prime}\right)_{k} \frac{\partial}{\partial x_{k}} \\
& =\sum_{k=1}^{n}\left[\left(\nabla_{\gamma^{\prime}}^{2} \tau(\gamma)\right)_{k}+\left(\nabla_{\left(\nabla_{\gamma^{\prime}} \tau(\gamma)\right)} \gamma^{\prime}\right)_{k}-\left(\nabla_{\left(\nabla_{\tau_{\gamma}} \gamma^{\prime}\right)} \gamma^{\prime}\right)_{k}-\left(\nabla_{\gamma^{\prime}} \nabla_{\tau(\gamma)} \gamma^{\prime}\right)_{k}\right. \\
& \left.+\left(\nabla_{\tau(\gamma)} \nabla_{\gamma^{\prime}} \gamma^{\prime}\right)_{k}\right] \frac{\partial}{\partial x_{k}} .
\end{aligned}
$$

Indicando por $\tau_{l}$ a $l$-ésima coordenada de $\tau(\gamma)$, valem as expressões:

$$
\begin{aligned}
\left(\nabla_{\gamma^{\prime}}^{2} \tau(\gamma)\right)_{k} & =\frac{d}{d t}\left(\nabla_{\gamma^{\prime}} \tau(\gamma)\right)_{k}+\sum_{i, j=1}^{n} \Gamma_{i j}^{k}\left(\nabla_{\gamma^{\prime}} \tau(\gamma)\right)_{i} \gamma_{j}^{\prime} \\
\left(\nabla_{\left(\nabla_{\gamma^{\prime}} \tau(\gamma)\right)} \gamma^{\prime}\right)_{k} & =\sum_{i, j=1}^{n} \Gamma_{i j}^{k} \gamma_{i}^{\prime}\left(\nabla_{\gamma^{\prime}} \tau(\gamma)\right)_{j} \\
\left(\nabla_{\left(\nabla_{\tau(\gamma)} \gamma^{\prime}\right)} \gamma^{\prime}\right)_{k} & =\sum_{i, j=1}^{n} \Gamma_{i j}^{k} \gamma_{i}^{\prime}\left(\nabla_{\tau(\gamma)} \gamma^{\prime}\right)_{j} \\
\left(\nabla_{\gamma^{\prime}} \nabla_{\tau(\gamma)} \gamma^{\prime}\right)_{k} & =\frac{d}{d t}\left(\nabla_{\tau(\gamma)} \gamma^{\prime}\right)_{k}+\sum_{i, j=1}^{n} \Gamma_{i j}^{k}\left(\nabla_{\tau(\gamma)} \gamma^{\prime}\right)_{i} \gamma_{j}^{\prime} \\
\left(\nabla_{\tau(\gamma)} \nabla_{\gamma^{\prime}} \gamma^{\prime}\right)_{k} & =\sum_{l}^{n} \tau_{l} \frac{\partial \tau_{k}}{\partial x_{l}}+\sum_{i, j}^{n} \Gamma_{i j}^{k} \tau_{i} \tau_{j}
\end{aligned}
$$

onde,

$$
\begin{gathered}
\nabla_{\gamma^{\prime}} \tau(\gamma)=\sum_{k=1}^{n}\left[\frac{d}{d t} \tau_{k}+\sum_{i, j}^{n} \Gamma_{i j}^{k} \tau_{i} \gamma_{j}^{\prime}\right] \frac{\partial}{\partial x_{k}}, \\
\nabla_{\tau(\gamma)} \gamma^{\prime}=\sum_{k=1}^{n}\left[\sum_{l=1}^{n} \tau_{l} \frac{\partial \gamma_{k}^{\prime}}{\partial x_{l}}+\sum_{i, j}^{n} \Gamma_{i, j}^{k} \gamma_{i}^{\prime} \tau_{j}\right] \frac{\partial}{\partial x_{k}} .
\end{gathered}
$$

Portanto, obtemos a expressão desejada:

$$
\begin{aligned}
\left(\tau_{2}(\gamma)\right)_{k} & =\frac{d}{d t}\left(\nabla_{\gamma^{\prime}} \tau(\gamma)\right)_{k}+2 \sum_{i, j=1}^{n} \Gamma_{i j}^{k} \gamma_{i}^{\prime}\left(\nabla_{\gamma^{\prime}} \tau(\gamma)\right)_{j}-\frac{d}{d t}\left(\nabla_{\tau(\gamma)} \gamma^{\prime}\right)_{k}-2 \sum_{i, j=1}^{n} \Gamma_{i j}^{k} \gamma_{i}^{\prime}\left(\nabla_{\tau(\gamma)} \gamma^{\prime}\right)_{j} \\
& +\sum_{l}^{n} \tau_{l} \frac{\partial \tau_{k}}{\partial x_{l}}+\sum_{i, j}^{n} \Gamma_{i j}^{k} \tau_{i} \tau_{j} .
\end{aligned}
$$




\section{Capítulo 2}

\section{Estudo das curvas bi-harmônicas com o método de Euler-Lagrange}

O propósito deste capítulo é expor um método alternativo, ao obtido no Capítulo 1, para descrever as curvas bi-harmônicas de uma variedade Riemanniana.

Observe que no caso das curvas harmônicas (i.e. as geodésicas) vale o seguinte resultado:

Teorema 2.0.1. Para o cálculo de geodésicas em uma variedade Riemanniana $\left(M^{n}, g\right)$, os seguintes sistemas são equivalentes:

$$
\begin{array}{ll}
x_{k}^{\prime \prime}+\sum_{i, j=1}^{n} \Gamma_{i j}^{k} x_{i}^{\prime} x_{j}^{\prime}=0, & k=1, \ldots, n . \\
\frac{d}{d t}\left(\frac{\partial L}{\partial x_{k}^{\prime}}\right)-\frac{\partial L}{\partial x_{k}}=0, & k=1, \ldots, n,
\end{array}
$$

Aqui

$$
L=\frac{1}{2} \sum_{i, j=1}^{n} g_{i j} x_{i}^{\prime} x_{j}^{\prime}
$$

é a Lagrangiana nas coordenadas locais $\left\{x_{i}\right\}$ de $M$.

Demonstração. Como

$$
L\left(x(t), x^{\prime}(t)\right)=\frac{1}{2} \sum_{i, j} g_{i j}(x(t)) x_{i}^{\prime} x_{j}^{\prime}
$$

então

$$
\frac{\partial L}{\partial x_{k}}=\frac{1}{2} \sum_{i, j=1}^{n} \frac{\partial g_{i j}}{\partial x_{k}} x_{i}^{\prime} x_{j}^{\prime}, \quad \frac{\partial L}{\partial x_{k}^{\prime}}=\sum_{i=1}^{n} g_{k i} x_{i}^{\prime}
$$


e

$$
\frac{d}{d t}\left(\frac{\partial L}{\partial x_{k}^{\prime}}\right)=\sum_{i=1}^{n} g_{k i} x_{i}^{\prime \prime}+\sum_{i, j=1}^{n} \frac{\partial g_{k i}}{\partial x_{j}} x_{i}^{\prime} x_{j}^{\prime}
$$

Portanto

$$
\begin{aligned}
\frac{d}{d t}\left(\frac{\partial L}{\partial x_{k}^{\prime}}\right)-\frac{\partial L}{\partial x_{k}} & =\sum_{i=1}^{n} g_{k i} x_{i}^{\prime \prime}+\sum_{i, j=1}^{n}\left(\frac{\partial g_{k i}}{\partial x_{j}}-\frac{1}{2} \frac{\partial g_{i j}}{\partial x_{k}}\right) x_{i}^{\prime} x_{j}^{\prime} \\
& =\sum_{i=1}^{n} g_{k i} x_{i}^{\prime \prime}+\frac{1}{2} \sum_{i, j=1}^{n}\left(\frac{\partial g_{k i}}{\partial x_{j}}+\frac{\partial g_{k j}}{\partial x_{i}}-\frac{\partial g_{i j}}{\partial x_{k}}\right) x_{i}^{\prime} x_{j}^{\prime} \\
& =\sum_{i=1}^{n} g_{k i} x_{i}^{\prime \prime}+\frac{1}{2} \sum_{i, j=1}^{n}\left(\frac{\partial g_{i k}}{\partial x_{j}}+\frac{\partial g_{k j}}{\partial x_{i}}-\frac{\partial g_{i j}}{\partial x_{k}}\right) x_{i}^{\prime} x_{j}^{\prime}
\end{aligned}
$$

Usando a condição de que a expressão acima deve se anular e multiplicando por $g^{i k}$, obtemos

$$
x_{k}^{\prime \prime}+\sum_{i, j=1}^{n} \frac{1}{2} \sum_{l=1}^{n} g^{l k}\left(\frac{\partial g_{i k}}{\partial x_{j}}+\frac{\partial g_{k j}}{\partial x_{i}}-\frac{\partial g_{i j}}{\partial x_{k}}\right) x_{i}^{\prime} x_{j}^{\prime}=0
$$

O resultado segue da expressão dos símbolos de Christoffel.

Motivados por este resultado, R. Caddeo, S. Montaldo, C. Oniciuc e P. Piu propuseram, em [10], um método baseado na idéia que as curvas bi-harmônicas $\gamma:(a, b) \rightarrow\left(M^{n}, g\right)$ são pontos críticos do funcional bi-energia

$$
E_{2}=\int_{a}^{b} L_{2}\left(x(t), x^{\prime}(t), x^{\prime \prime}(t)\right) d t
$$

para uma oportuna bi-lagrangiana $L_{2}$, que depende das componentes locais de $\gamma$ e de suas derivadas até a segunda ordem. Portanto, da teoria clássica de Euler-Lagrange, segue que as curvas bi-harmônicas são soluções do sistema

$$
\frac{d^{2}}{d t^{2}}\left(\frac{\partial L_{2}}{\partial x_{k}^{\prime \prime}}\right)-\frac{d}{d t}\left(\frac{\partial L_{2}}{\partial x_{k}^{\prime}}\right)+\frac{\partial L_{2}}{\partial x_{k}}=0, \quad k=1, \ldots, n
$$

onde $\gamma(t)=\left(x_{1}(t), \ldots, x_{n}(t)\right)$ representa a expressão da curva $\gamma$ com respeito as coordenadas locais $\left\{x_{i}\right\}$ de $M$.

Na Seção 2.1 provaremos a equivalência dos dois métodos, ou seja, entre a equação biharmônica (1.46) e o sistema (2.4). Em seguida, o método de Euler-Lagrange é aplicado para determinar as curvas bi-harmônicas do plano hiperbólico e do grupo de Lie Sol.

\subsection{Equivalência dos dois métodos}

Seja $\gamma:(a, b) \rightarrow(M, g)$ uma curva diferenciável cuja expressão local é dada por $\gamma(t)=$ $\left(x_{1}(t), \ldots, x_{n}(t)\right)$. Nesta seção mostraremos a equivalência entre a equação bi-harmônica (1.46) 
e o sistema de Euler-Lagrange (2.4). Precisaremos do seguinte

Lema 2.1.1. Seja $\gamma:(a, b) \rightarrow(M, g)$ uma curva diferenciável e $T=\gamma^{\prime}$ seu campo de vetores velocidade. Sejam $\tau_{i}=x_{i}^{\prime \prime}+\sum_{j, k} \Gamma_{j k}^{i} x_{j}^{\prime} x_{k}^{\prime}$ as componentes do campo de tensão de $\gamma$ com respeito ao sistema de coordenadas locas $\left\{x_{i}\right\}$ de $M$ e

$$
L_{2}=\frac{1}{2} \sum_{i, j} g_{i j}(x(t)) \tau_{i} \tau_{j}
$$

a expressão da bi-lagrangiana nas coordenadas locais. Então

$$
\begin{aligned}
& \text { (i) } \frac{\partial L_{2}}{\partial x_{k}}=g\left(\nabla_{T}^{2} \frac{\partial}{\partial x_{k}}, \tau(\gamma)\right)+g\left(\mathrm{R}\left(T, \frac{\partial}{\partial x_{k}}\right) T, \tau(\gamma)\right) \\
& \text { (ii) } \frac{d}{d t}\left(\frac{\partial L_{2}}{\partial x_{k}^{\prime}}\right)=2 g\left(\nabla_{T}^{2} \frac{\partial}{\partial x_{k}}, \tau(\gamma)\right)+2 g\left(\nabla_{T} \frac{\partial}{\partial x_{k}}, \nabla_{T} \tau(\gamma)\right) \\
& (\text { iii }) \frac{d^{2}}{d t^{2}}\left(\frac{\partial L_{2}}{\partial x_{k}^{\prime \prime}}\right)=g\left(\nabla_{T}^{2} \tau(\gamma), \frac{\partial}{\partial x_{k}}\right)+2 g\left(\nabla_{T} \tau(\gamma), \nabla_{T} \frac{\partial}{\partial x_{k}}\right)+g\left(\tau(\gamma), \nabla_{T}^{2} \frac{\partial}{\partial x_{k}}\right) .
\end{aligned}
$$

Demonstração. A partir de agora passaremos a omitir o símbolo de somatória em nossos cálculos, para que a notação não fique sobrecarregada.

(i) Da expressão da bi-lagrangiana temos

$$
\frac{\partial L_{2}}{\partial x_{k}}=\left(\frac{1}{2} \frac{\partial g_{i j}}{\partial x_{k}} \tau_{i} \tau_{j}+g_{i j} \frac{\partial \tau_{i}}{\partial x_{k}} \tau_{j}\right) .
$$

Também, usando a fórmula de Koszul para $g_{i j}$ e a expressão de $\tau(\gamma)$, resulta que

$$
\frac{\partial L_{2}}{\partial x_{k}}=g_{i j} \tau_{j}\left\{\Gamma_{k a}^{i} x_{a}^{\prime \prime}+\left(\Gamma_{k c}^{i} \Gamma_{b a}^{c}+\frac{\partial \Gamma_{a b}^{i}}{\partial x_{k}}\right) x_{a}^{\prime} x_{b}^{\prime}\right\} .
$$

Agora, de

$$
\Gamma_{k a}^{i} x_{a}^{\prime \prime}=\left(\nabla_{T}^{2} \frac{\partial}{\partial x_{k}}\right)_{i}-\left(\Gamma_{a c}^{i} \Gamma_{b k}^{c}+\frac{\partial \Gamma_{b k}^{i}}{\partial x_{a}}\right) x_{a}^{\prime} x_{b}^{\prime}
$$

obtemos

$$
\begin{aligned}
\frac{\partial L_{2}}{\partial x_{k}} & =g_{i j} \tau_{j}\left\{\left(\nabla_{T}^{2} \frac{\partial}{\partial x_{k}}\right)_{i}+x_{a}^{\prime} x_{b}^{\prime} \mathrm{R}_{b k a}^{i}\right\} \\
& =g\left(\nabla_{T}^{2} \frac{\partial}{\partial x_{k}}, \tau(\gamma)\right)+g\left(\mathrm{R}\left(T, \frac{\partial}{\partial x_{k}}\right) T, \tau(\gamma)\right) .
\end{aligned}
$$

(ii) Como

$$
\begin{aligned}
\frac{\partial L_{2}}{\partial x_{k}^{\prime}} & =g_{i j} \frac{\partial \tau_{i}}{\partial x_{k}^{\prime}} \tau_{j} \\
& =g_{i j} \tau_{j}\left(\frac{\partial x_{i}^{\prime \prime}}{\partial x_{k}^{\prime}}+2 \Gamma_{b k}^{i} x_{b}^{\prime}\right) \\
& =2 g\left(\nabla_{T} \frac{\partial}{\partial x_{k}}, \tau(\gamma)\right),
\end{aligned}
$$


então

$$
\frac{d}{d t}\left(\frac{\partial L_{2}}{\partial x_{k}^{\prime}}\right)=2 g\left(\nabla_{T}^{2} \frac{\partial}{\partial x_{k}}, \tau(\gamma)\right)+2 g\left(\nabla_{T} \frac{\partial}{\partial x_{k}}, \nabla_{T} \tau(\gamma)\right)
$$

(iii) De forma análoga ao feito no ítem anterior, sendo

$$
\frac{\partial L_{2}}{\partial x_{k}^{\prime \prime}}=g_{i j} \frac{\partial \tau_{i}}{\partial x_{k}^{\prime \prime}} \tau_{j} \quad \text { e } \quad \frac{\partial \tau_{i}}{\partial x_{k}^{\prime \prime}}=\delta_{k i}
$$

temos

$$
\frac{\partial L_{2}}{\partial x_{k}^{\prime \prime}}=g_{k j} \tau_{j}=g\left(\tau(\gamma), \frac{\partial}{\partial x_{k}}\right)
$$

Logo

$$
\frac{d^{2}}{d t^{2}}\left(\frac{\partial L_{2}}{\partial x_{k}^{\prime \prime}}\right)=g\left(\nabla_{T}^{2} \tau(\gamma), \frac{\partial}{\partial x_{k}}\right)+2 g\left(\nabla_{T} \tau(\gamma), \nabla_{T} \frac{\partial}{\partial x_{k}}\right)+g\left(\tau(\gamma), \nabla_{T}^{2} \frac{\partial}{\partial x_{k}}\right),
$$

o que termina a prova.

Temos o seguinte resultado:

Proposição 2.1.2. Seja $\gamma:(a, b) \subset \mathbb{R} \rightarrow(M, g)$ uma curva diferenciável e $T=\gamma^{\prime}$ seu campo de vetores velocidade. Então a equação

$$
\nabla_{T}^{3} T+R\left(T, \nabla_{T} T\right) T=0
$$

é equivalente ao sistema

$$
\frac{d^{2}}{d t^{2}}\left(\frac{\partial L_{2}}{\partial x_{k}^{\prime \prime}}\right)-\frac{d}{d t}\left(\frac{\partial L_{2}}{\partial x_{k}^{\prime}}\right)+\frac{\partial L_{2}}{\partial x_{k}}=0, \quad k=1, \ldots, n
$$

onde

$$
L_{2}\left(x(t), x^{\prime}(t), x^{\prime \prime}(t)\right)=\frac{1}{2} g(\tau(\gamma)(\gamma), \tau(\gamma)(\gamma))
$$

Demonstração. Do Lema 2.1.1 e do fato que $\tau(\gamma)=\nabla_{T} T$, temos

$$
\begin{aligned}
\frac{d^{2}}{d t^{2}}\left(\frac{\partial L_{2}}{\partial x_{k}^{\prime \prime}}\right)-\frac{d}{d t}\left(\frac{\partial L_{2}}{\partial x_{k}^{\prime}}\right)+\frac{\partial L_{2}}{\partial x_{k}} & =g\left(\nabla_{T}^{2} \tau(\gamma), \frac{\partial}{\partial x_{k}}\right)+2 g\left(\nabla_{T} \tau(\gamma), \nabla_{T} \frac{\partial}{\partial x_{k}}\right)+g\left(\tau(\gamma), \nabla_{T}^{2} \frac{\partial}{\partial x_{k}}\right) \\
& -2 g\left(\nabla_{T}^{2} \frac{\partial}{\partial x_{k}}, \tau(\gamma)\right)-2 g\left(\nabla_{T} \frac{\partial}{\partial x_{k}}, \nabla_{T} \tau(\gamma)\right) \\
& +g\left(\nabla_{T}^{2} \frac{\partial}{\partial x_{k}}, \tau(\gamma)\right)+g\left(\mathrm{R}\left(T, \frac{\partial}{\partial x_{k}}\right) T, \tau(\gamma)\right) \\
& =g\left(\nabla_{T}^{3} T+\mathrm{R}\left(T, \nabla_{T} T\right) T, \frac{\partial}{\partial x_{k}}\right)
\end{aligned}
$$


Observação 2.1.3. Recordamos que a Lagrangiana $L$ de primeira ordem, ou seja quando $L$ depende apenas de $t, x(t)$ e $x^{\prime}(t)$, corresponde a "energia natural" dada por:

$$
\mathfrak{E}=\sum_{i} x_{i}^{\prime} \frac{\partial L}{\partial x_{i}^{\prime}}-L
$$

A característica principal de $\mathfrak{E}$ é de ser constante ao longo de soluções do sistema de EulerLagrange associado à Lagrangiana $L$. De fato:

$$
\begin{aligned}
\frac{d}{d t} \mathfrak{E}(\gamma) & =\sum_{i}\left[x_{i}^{\prime \prime} \frac{\partial L}{\partial x_{i}^{\prime}}+x_{i}^{\prime} \frac{d}{d t}\left(\frac{\partial L}{\partial x_{i}^{\prime}}\right)-\frac{\partial L}{\partial x_{i}} x_{i}^{\prime}-\frac{\partial L}{\partial x_{i}^{\prime}} x_{i}^{\prime \prime}\right] \\
& =\sum_{i} x_{i}^{\prime}\left[\frac{d}{d t}\left(\frac{\partial L}{\partial x_{i}^{\prime}}\right)-\frac{\partial L}{\partial x_{i}}\right]=0 .
\end{aligned}
$$

No caso da bi-lagragiana $L_{2}$, que depende das derivadas das componentes da curva até a segunda ordem, a "bi-energia" natural, definida ao longo de $\gamma$, é

$$
\mathfrak{E}_{2}(\gamma)=\sum_{k}\left\{x_{k}^{\prime}\left(\frac{\partial L_{2}}{\partial x_{k}^{\prime}}-\frac{d}{d t} \frac{\partial L_{2}}{\partial x_{k}^{\prime \prime}}\right)+x_{k}^{\prime \prime} \frac{\partial L_{2}}{\partial x_{k}^{\prime \prime}}\right\}-L_{2}
$$

Cabe aqui observar que, usando o Lema 2.1.1, podemos reescrever $\mathfrak{E}$ na forma

$$
\mathfrak{E}_{2}=\frac{1}{2}\|\tau(\gamma)\|^{2}-g\left(\gamma^{\prime}, \nabla_{\gamma^{\prime}} \tau(\gamma)\right)
$$

Passamos a demonstrar que $\mathfrak{E}_{2}$ é constante ao longo de uma solução do respectivo sistema de Euler-Lagrange (2.6). Com efeito

$$
\begin{aligned}
\frac{d}{d t} \mathfrak{E}_{2}(\gamma) & =\sum_{k}\left\{x_{k}^{\prime \prime}\left(\frac{\partial L_{2}}{\partial x_{k}^{\prime}}-\frac{d}{d t}\left(\frac{\partial L_{2}}{\partial x_{k}^{\prime \prime}}\right)\right)+x_{k}^{\prime}\left(\frac{d}{d t}\left(\frac{\partial L_{2}}{\partial x_{k}^{\prime}}\right)-\frac{d^{2}}{d t^{2}}\left(\frac{\partial L_{2}}{\partial x_{k}^{\prime \prime}}\right)\right)+x_{k}^{\prime \prime \prime} \frac{\partial L_{2}}{\partial x_{k}^{\prime \prime}}\right. \\
& \left.+x_{k}^{\prime \prime} \frac{d}{d t}\left(\frac{\partial L_{2}}{\partial x_{k}^{\prime \prime}}\right)-\frac{\partial L_{2}}{\partial x_{k}} x_{k}^{\prime}-\frac{\partial L_{2}}{\partial x_{k}^{\prime}} x_{k}^{\prime \prime}-\frac{\partial L_{2}}{\partial x_{k}^{\prime \prime}} x_{k}^{\prime \prime \prime}\right\} \\
& =-\sum_{k}\left\{x_{k}^{\prime}\left(\frac{d^{2}}{d t^{2}}\left(\frac{\partial L_{2}}{\partial x_{k}^{\prime \prime}}\right)-\frac{d}{d t}\left(\frac{\partial L_{2}}{\partial x_{k}^{\prime}}\right)+\frac{\partial L_{2}}{\partial x_{k}}\right)\right\}=0 .
\end{aligned}
$$

Como consequência da Observação 2.1.3, resulta a

Proposição 2.1.4. Seja $\gamma: I \rightarrow(M, g)$ uma curva bi-harmônica parametrizada pelo comprimento de arco. Então a bi-Lagragiana $L_{2}=\frac{1}{2} g(\tau(\gamma), \tau(\gamma))$ é constante ao longo de $\gamma$.

Demonstração. Derivando duas vezes $g\left(\gamma^{\prime}, \gamma^{\prime}\right)=1$ temos

$$
g\left(\nabla_{\gamma^{\prime}} \tau(\gamma), \gamma^{\prime}\right)=-g(\tau(\gamma), \tau(\gamma))
$$


Substituindo (2.8) em (2.7) resulta que

$$
\mathfrak{E}_{2}=\frac{3}{2}\|\tau(\gamma)\|^{2}
$$

Logo, se $\gamma$ é bi-harmônica, $\mathfrak{E}_{2}$ é constante ao longo de $\gamma$ e, portanto, $L_{2}$ também o é.

Observação 2.1.5. Pondo

$$
a:=\frac{1}{2}\|\tau(\gamma)\|^{2}-g\left(\gamma^{\prime}, \nabla_{\gamma^{\prime}} \tau(\gamma)\right) \quad \text { e } \quad b:=\left\|\nabla_{\gamma^{\prime}} \gamma^{\prime}\right\|^{2}
$$

verifica-se facilmente que se uma curva bi-harmônica $\gamma(t)$ continua sendo bi-harmônica após uma reparametrização $s(t)$ que a torne de velocidade unitária, então a função $s(t)$ deve satisfazer a EDO:

$$
2 s^{\prime} s^{\prime \prime}-s^{\prime \prime 2}-3 b s^{\prime 4}+2 a=0 .
$$

Observe também, que um polinômio $P^{n}(t)$ é uma solução desta equação se e somente se, para $b \neq 0$ e $a>0$,

$$
s(t)=P^{n}(t)=c+\sqrt[4]{\frac{2 a}{3 b}} t, \quad c \in \mathbb{R} .
$$

Nos exemplos que seguem passamos a considerar curvas bi-harmônicas parametrizadas pelo comprimento de arco.

Exemplo 2.1.6 (Curvas bi-harmônicas em $\mathbb{R}^{3}$ ). Seja $\gamma(t)=(x(t), y(t), z(t))$ uma curva em $\mathbb{R}^{3}$ parametrizada pelo comprimento de arco, então seu campo de tensão é $\tau(\gamma)(t)=\left(\tau_{1}, \tau_{2}, \tau_{3}\right)=$ $\left(x^{\prime \prime}, y^{\prime \prime}, z^{\prime \prime}\right)$ e o sistema (2.6), para a bi-lagragiana

$$
L_{2}=\frac{1}{2}\left(x^{\prime \prime 2}+y^{\prime \prime 2}+z^{\prime \prime 2}\right)
$$

se reduz a

$$
\left\{\begin{array}{l}
x^{(i v)}=0 \\
y^{(i v)}=0 \\
z^{(i v)}=0 .
\end{array}\right.
$$

As soluções gerais de (2.9) são polinômios de grau três no parâmetro $t$. Se olharmos para soluções parametrizadas pelo comprimento de arco, o grau deverá ser 1, o que implica no fato de as curvas serem geodésicas (i.e., retas de $\mathbb{R}^{3}$ ).

Exemplo 2.1.7 (Curvas bi-harmônicas em $\mathbb{H}^{2}$ ). Neste exemplo vamos determinar as curvas bi-harmônicas do plano hiperbólico $\mathbb{H}^{2}$, provando que as mesmas são geodésicas. 
Considere o modelo do semi-plano superior do plano hiperbólico $\mathbb{H}^{2}=\left\{(x, y) \in \mathbb{R}^{2} \mid y>0\right\}$ munido da métrica

$$
g=\frac{d x^{2}+d y^{2}}{y^{2}}
$$

Temos o seguinte resultado:

Teorema 2.1.8. Seja $\gamma: I \rightarrow\left(\mathbb{H}^{2}, g\right)$ uma curva diferenciável parametrizada pelo comprimento de arco. Então $\gamma$ é bi-harmônica se e somente se sua curvatura geodésica satisfaz o sistema

$$
\left\{\begin{array}{l}
\kappa_{g}^{\prime \prime}-\kappa_{g}^{3}-\kappa_{g}=0 \\
\kappa_{g} \kappa_{g}^{\prime}=0
\end{array}\right.
$$

Demonstração. Os símbolos de Christoffel de $\left(\mathbb{H}^{2}, g\right)$ não nulos são

$$
\Gamma_{12}^{1}=\Gamma_{21}^{1}=\Gamma_{22}^{2}=-\frac{1}{y}, \quad \Gamma_{11}^{2}=\frac{1}{y},
$$

e as componentes do campo de tensão de uma curva genérica $\gamma$ de $\mathbb{H}^{2}$ são dadas por:

$$
\tau_{1}=x^{\prime \prime}-2 \frac{x^{\prime} y^{\prime}}{y} \quad \text { e } \quad \tau_{2}=y^{\prime \prime}+\frac{x^{\prime 2}-y^{\prime 2}}{y} .
$$

Além disso, a função bi-lagrangiana é

$$
L_{2}=\frac{1}{2 y^{2}}\left(\tau_{1}^{2}+\tau_{2}^{2}\right)
$$

e o sistema de Euler-Lagrange assume a forma que segue:

$$
\left\{\begin{array}{l}
\frac{d}{d t}\left(\frac{1}{y^{2}} \frac{d}{d t}\left(\tau_{1}\right)-\frac{2 x^{\prime} \tau_{2}}{y^{3}}\right)=0 \\
\frac{d^{2}}{d t^{2}}\left(\frac{\tau(\gamma)^{2}}{y^{2}}\right)+\frac{d}{d t}\left(2 \frac{x^{\prime} \tau_{1}+y^{\prime} \tau_{2}}{y^{3}}\right)-\frac{\tau_{1}^{2}+\tau_{2}^{2}}{y^{3}}+2 \frac{x^{\prime} y^{\prime}}{y^{4}} \tau_{1}-\frac{x^{\prime 2}-y^{\prime 2}}{y^{4}} \tau_{2}=0
\end{array}\right.
$$

Sendo que o sistema acima não é de fácil integração, faremos algumas considerações de acordo com a tese [30]. Da definição de curvatura geodésica obtemos que

$$
\kappa_{g}^{2}=\frac{\tau_{1}^{2}+\tau_{2}^{2}}{y^{2}},
$$

logo, (ver [6], pg 543),

$$
\kappa_{g}=\frac{y^{\prime} \tau_{1}-x^{\prime} \tau_{2}}{y^{2}}
$$

Desta última segue que

$$
\kappa_{g}^{\prime}=\frac{y^{\prime} \frac{d}{d t}\left(\tau_{1}\right)-x^{\prime} \frac{d}{d t}\left(\tau_{2}\right)}{y^{2}}-\frac{\tau_{1}}{y} .
$$


Derivando $\kappa_{g}^{2}$ temos

$$
\kappa_{g} \kappa_{g}^{\prime}=\frac{\tau_{1} \frac{d}{d t}\left(\tau_{1}\right)+\tau_{2} \frac{d}{d t}\left(\tau_{2}\right)}{y^{2}}-\frac{y^{\prime}}{y} \kappa_{g}^{2}
$$

e, derivando $\kappa_{g}^{\prime}$, resulta que

$$
\kappa_{g}^{\prime \prime}=\frac{y^{\prime} \frac{d^{2}}{d t^{2}}\left(\tau_{1}\right)-x^{\prime} \frac{d^{2}}{d t^{2}}\left(\tau_{2}\right)}{y^{2}}+\frac{\tau_{2} \frac{d}{d t}\left(\tau_{1}\right)-\tau_{1} \frac{d}{d t}\left(\tau_{2}\right)}{y^{2}}-\frac{2}{y} \frac{d}{d t}\left(\tau_{1}\right)+\frac{y^{\prime}}{y^{2}}\left(\tau_{1}\right) .
$$

Como $\gamma$ é parametrizada pelo comprimento de arco, vale a condição

$$
\frac{x^{\prime 2}+y^{\prime 2}}{y^{2}}=1
$$

a qual, por dupla derivação, fornece

$$
x^{\prime} \tau_{1}+y^{\prime} \tau_{2}=0
$$

e

$$
\kappa_{g}^{2}+\frac{x^{\prime}}{y} \kappa_{g}+\frac{x^{\prime} \frac{d}{d t}\left(\tau_{1}\right)+y^{\prime} \frac{d}{d t}\left(\tau_{2}\right)}{y^{2}}=0 .
$$

Pela (2.14), a equação (2.15) pode ser reescrita na forma

$$
\kappa_{g}^{2}-\frac{\tau_{2}}{y}+\frac{x^{\prime} \frac{d}{d t}\left(\tau_{1}\right)+y^{\prime} \frac{d}{d t}\left(\tau_{2}\right)}{y^{2}}=0 .
$$

Derivando (2.16), obtemos

$$
\begin{aligned}
& 2 \kappa_{g} \kappa_{g}^{\prime}+\frac{x^{\prime} \frac{d^{2}}{d t^{2}}\left(\tau_{1}\right)+y^{\prime} \frac{d^{2}}{d t^{2}}\left(\tau_{2}\right)}{y^{2}}+\frac{\tau_{1} \frac{d}{d t}\left(\tau_{1}\right)+\tau_{2} \frac{d}{d t}\left(\tau_{2}\right)}{y^{2}}+x^{\prime} \frac{y^{\prime} \frac{d}{d t}\left(\tau_{1}\right)-x^{\prime} \frac{d}{d t}\left(\tau_{2}\right)}{y^{3}} \\
- & y^{\prime} \frac{x^{\prime} \frac{d}{d t}\left(\tau_{1}\right)+y^{\prime} \frac{d}{d t}\left(\tau_{2}\right)}{y^{3}}-\frac{1}{y} \frac{d}{d t}\left(\tau_{2}\right)+\frac{y^{\prime}}{y^{2}} \tau_{2}=0 .
\end{aligned}
$$

A partir de algumas considerações e derivações, pode-se provar que o sistema de Euler-Lagrange pode ser reescrito na forma

$$
\left\{\begin{array}{l}
\frac{1}{y^{2}} \frac{d^{2}}{d t^{2}}\left(\tau_{1}\right)-2 \frac{\tau_{1} \tau_{2}}{y^{3}}-2 \frac{y^{\prime}}{y^{3}} \frac{d}{d t}\left(\tau_{1}\right)-2 \frac{x^{\prime}}{y^{3}} \frac{d}{d t}\left(\tau_{2}\right)+2 \frac{x^{\prime} y^{\prime}}{y^{4}} \tau_{2}=0 \\
\frac{1}{y^{2}} \frac{d^{2}}{d t^{2}}\left(\tau_{2}\right)-4 \frac{y^{\prime}}{y^{3}} \frac{d}{d t}\left(\tau_{2}\right)-\frac{\left(\tau_{1}\right)^{2}+3\left(\tau_{2}\right)^{2}}{y^{3}}+2 \frac{\left(y^{\prime}\right)^{2}}{y^{4}} \tau_{2}+\frac{\tau_{2}}{y^{2}}=0
\end{array}\right.
$$

Multiplicando a primeira equação por $x^{\prime}$, a segunda por $y^{\prime}$ e somando, resulta que

$$
\frac{x^{\prime} \frac{d^{2}}{d t^{2}}\left(\tau_{1}\right)+y^{\prime} \frac{d^{2}}{d t^{2}}\left(\tau_{2}\right)}{y^{2}}-\frac{y^{\prime}}{y} \kappa_{g}^{2}-2 y^{\prime} \frac{x^{\prime} \frac{d}{d t}\left(\tau_{1}\right)+y^{\prime} \frac{d}{d t}\left(\tau_{2}\right)}{y^{3}}-\frac{2}{y} \frac{d}{d t}\left(\tau_{2}\right)+3 \frac{y^{\prime}}{y^{2}} \tau_{2}=0 .
$$

Subtraindo esta última da (2.17) e usando (2.16), se tem

$$
2 \kappa_{g} \kappa_{g}^{\prime}+\frac{1}{y} \frac{d}{d t}\left(\tau_{2}\right)-\frac{y^{\prime}}{y^{2}} \tau_{2}+\frac{\tau_{1} \frac{d}{d t}\left(\tau_{1}\right)+\tau_{2} \frac{d}{d t}\left(\tau_{2}\right)}{y^{2}}+x^{\prime} \frac{y^{\prime} \frac{d}{d t}\left(\tau_{1}\right)-x^{\prime} \frac{d}{d t}\left(\tau_{2}\right)}{y^{3}}=0
$$


e, pela (2.12), resulta que

$$
3 \kappa_{g} \kappa_{g}^{\prime}+\frac{1}{y} \frac{d}{d t}\left(\tau_{2}\right)-\frac{y^{\prime}}{y^{2}} \tau_{2}+\frac{y^{\prime}}{y} \kappa_{g}^{2}+x^{\prime} \frac{y^{\prime} \frac{d}{d t}\left(\tau_{1}\right)-x^{\prime} \frac{d}{d t}\left(\tau_{2}\right)}{y^{3}}=0 .
$$

De (2.16) temos

$$
-\frac{1}{y} \frac{d}{d t}\left(\tau_{2}\right)+\frac{y^{\prime}}{y^{2}} \tau_{2}=\frac{y^{\prime}}{y} \kappa_{g}^{2}+x^{\prime} \frac{y^{\prime} \frac{d}{d t}\left(\tau_{1}\right)-x^{\prime} \frac{d}{d t}\left(\tau_{2}\right)}{y^{3}}
$$

portanto, de (2.19), segue que

$$
3 \kappa_{g} \kappa_{g}^{\prime}=0
$$

que é a segunda equação do sistema (2.10).

Consideramos novamente as equações do sistema (2.18); multiplicando a primeira delas por $y^{\prime}$, a segunda por $-x^{\prime}$ e somando, obtemos

$$
\frac{y^{\prime} \frac{d^{2}}{d t^{2}}\left(\tau_{1}\right)-x^{\prime} \frac{d^{2}}{d t^{2}}\left(\tau_{2}\right)}{y^{2}}+3 \frac{x^{\prime}}{y} \kappa_{g}^{2}-2 \frac{y^{\prime 2}}{y^{3}} \frac{d}{d t}\left(\tau_{1}\right)+2 \frac{x^{\prime} y^{\prime}}{y^{3}} \frac{d}{d t}\left(\tau_{2}\right)-\frac{x^{\prime}}{y^{2}}\left(\tau_{2}\right)=0
$$

da qual segue

$$
\frac{y^{\prime} \frac{d^{2}}{d t^{2}}\left(\tau_{1}\right)-x^{\prime} \frac{d^{2}}{d t^{2}}\left(\tau_{2}\right)}{y^{2}}+3 \frac{x^{\prime}}{y} \kappa_{g}^{2}-2 y^{\prime} \frac{y^{\prime} \frac{d}{d t}\left(\tau_{1}\right)-x^{\prime} \frac{d}{d t}\left(\tau_{2}\right)}{y^{3}}-\frac{x^{\prime}}{y^{2}}\left(\tau_{2}\right)=0 .
$$

Multiplicando (2.15) por $-\kappa_{g}$ e somando-a à equação (2.20), obtemos

$$
\frac{y^{\prime} \frac{d^{2}}{d t^{2}}\left(\tau_{1}\right)-x^{\prime} \frac{d^{2}}{d t^{2}}\left(\tau_{2}\right)}{y^{2}}+\frac{\tau_{2} \frac{d}{d t}\left(\tau_{1}\right)-\tau_{1} \frac{d}{d t}\left(\tau_{2}\right)}{y^{2}}-2 y^{\prime} \frac{\left(y^{\prime} \frac{d}{d t}\left(\tau_{1}\right)-x^{\prime} \frac{d}{d t}\left(\tau_{2}\right)\right)}{y^{3}}-\kappa^{3}+2 \frac{x^{\prime}}{y} \kappa_{g}^{2}-\frac{x^{\prime}}{y^{2}} \tau_{2}=0,
$$

da qual, usando (2.13), se deduz

$$
\kappa_{g}^{\prime \prime}-\kappa_{g}^{3}+\frac{2}{y} \frac{d}{d t}\left(\tau_{1}\right)-\frac{y^{\prime}}{y^{2}} \tau_{1}+2 \frac{x^{\prime}}{y} \kappa_{g}^{2}-2 y^{\prime} \frac{y^{\prime} \frac{d}{d t}\left(\tau_{1}\right)-x^{\prime} \frac{d}{d t}\left(\tau_{2}\right)}{y^{3}}-\frac{x^{\prime}}{y^{2}} \tau_{2}=0
$$

Por fim, multiplicamos (2.16) por $-2 \frac{x^{\prime}}{y}$, somamos ao resultado a (2.21) e utilizamos a expressão de $\kappa_{g}$ obtendo, assim, a primeira equação do sistema (2.10):

$$
\kappa_{g}^{\prime \prime}-\kappa_{g}^{3}-\kappa_{g}=0
$$

Quanto à classificação das curvas bi-harmônicas do plano hiperbólico, temos:

Corolário 2.1.9. Uma curva diferenciável $\gamma: I \rightarrow \mathbb{H}^{2}$ parametrizada pelo comprimento de arco é bi-harmônica se e somente se é uma geodésica. 
Demonstração. Da segunda equação de (2.10) resulta que $\kappa_{g}$ deve ser constante e, então, da primeira equação segue que $\kappa_{g}$ deve ser nula.

Exemplo 2.1.10 (Curvas bi-harmônicas do espaço Sol). Neste exemplo, usamos o sistema (2.6) para caracterizar as curvas bi-harmônicas do grupo de Lie tridimensional Sol. Representaremos o espaço Sol como sendo o grupo de matrizes

$$
\left\{\left(\begin{array}{ccc}
e^{-x} & 0 & y \\
0 & e^{x} & z \\
0 & 0 & 1
\end{array}\right): x, y, z \in \mathbb{R}\right\},
$$

munido da métrica invariante à esquerda, dada por

$$
g=d x^{2}+e^{2 x} d y^{2}+e^{-2 x} d z^{2} .
$$

Com respeito a esta métrica, uma base ortonormal de campos invariantes à esquerda é

$$
E_{1}=\frac{\partial}{\partial x}, \quad E_{2}=e^{-x} \frac{\partial}{\partial y}, \quad E_{3}=e^{x} \frac{\partial}{\partial z}
$$

Dada uma curva parametrizada pelo comprimento de arco $\gamma(t)=(x(t), y(t), z(t))$, iremos decompor seu campo de vetores velocidade na base (2.22):

$$
T=\sum_{i} T_{i} E_{i}=x^{\prime} E_{1}+e^{x} y^{\prime} E_{2}+e^{-x} z^{\prime} E_{3}
$$

Então, o campo de tensão de $\gamma$ pode ser escrito como

$$
\tau(\gamma)=\nabla_{T} T=\tau_{i} E_{i}
$$

onde

$$
\left\{\begin{aligned}
\tau_{1} & =x^{\prime \prime}-e^{2 x} y^{\prime 2}+e^{-2 x} z^{\prime 2} \\
\tau_{2} & =e^{x} y^{\prime \prime}+2 e^{x} x^{\prime} y^{\prime} \\
\tau_{3} & =e^{-x} z^{\prime \prime}-2 e^{-x} x^{\prime} z^{\prime}
\end{aligned}\right.
$$

Com isso, o sistema de Euler-Lagrange (2.6) se torna

$$
\left\{\begin{array}{l}
\frac{d^{2} \tau_{1}}{d t^{2}}-\frac{d}{d t}\left(2 e^{x} y^{\prime} \tau_{2}-2 e^{-x} z^{\prime} \tau_{3}\right)-\tau_{1}\left(2 e^{2 x} y^{\prime 2}+2 e^{-2 x} z^{\prime 2}\right)+\tau_{2}^{2}-\tau_{3}^{2}=0 \\
\frac{d^{2}}{d t^{2}}\left(e^{x} \tau_{2}\right)-\frac{d}{d t}\left(-2 e^{2 x} y^{\prime} \tau_{1}+2 e^{x} x^{\prime} \tau_{2}\right)=0 \\
\frac{d^{2}}{d t^{2}}\left(e^{-x} \tau_{3}\right)-\frac{d}{d t}\left(2 e^{-2 x} z^{\prime} \tau_{1}-2 e^{-x} x^{\prime} \tau_{3}\right)=0 .
\end{array}\right.
$$

Mostraremos agora que o sistema (2.23) implica a seguinte 
Proposição 2.1.11. As curvas bi-harmônicas parametrizadas pelo comprimento de arco do espaço Sol são as geodésicas.

Demonstração. Derivando duas vezes $g\left(\gamma^{\prime}, \gamma^{\prime}\right)=1$ temos

$$
\begin{aligned}
& \left(x^{\prime \prime}\right)^{2}+x^{\prime} x^{\prime \prime \prime}+2 e^{2 x}\left(x^{\prime}\right)^{2}\left(y^{\prime}\right)^{2}+e^{2 x} x^{\prime \prime}\left(y^{\prime}\right)^{2}+4 e^{2 x} x^{\prime} y^{\prime} y^{\prime \prime}+e^{2 x}\left(y^{\prime \prime}\right)^{2} \\
+ & e^{2 x} y^{\prime} y^{\prime \prime \prime}+2 e^{-2 x} x^{\prime \prime}\left(z^{\prime}\right)^{2}-4 e^{-2 x} x^{\prime} z^{\prime} z^{\prime \prime}+e^{-2 x}\left(z^{\prime \prime}\right)^{2}+e^{-2 x} z^{\prime} z^{\prime \prime \prime}=0 .
\end{aligned}
$$

A derivação de (2.24) com respeito a $y^{\prime \prime}$ e $z^{\prime \prime}$ nos dá

$$
\left\{\begin{array}{l}
2 e^{2 x}\left(y^{\prime \prime}+2 x^{\prime} y^{\prime}\right)=2 e^{x} \tau_{2}=0 \\
2 e^{-2 x}\left(z^{\prime \prime}-2 x^{\prime} z^{\prime}\right)=2 e^{-x} \tau_{3}=0,
\end{array}\right.
$$

o que implica $\tau_{2}=\tau_{3}=0$. Além disso, de

$$
g(T, \tau(\gamma))=\frac{1}{2} \frac{d}{d t} g(T, T)=0
$$

deduzimos que $T_{1}=x^{\prime}=0$ e, então,

$$
\left\{\begin{array}{l}
\tau_{1}=-e^{2 x} y^{\prime 2}+e^{-2 x} z^{2} \\
\tau_{2}=e^{x} y^{\prime \prime}=0 \\
\tau_{3}=e^{-x} z^{\prime \prime}=0
\end{array}\right.
$$

Pondo $e^{x} y^{\prime}=a$ e $e^{-x} z^{\prime}=b$, com $a, b \in \mathbb{R}$, obtemos $\tau_{1}=\left(b^{2}-a^{2}\right)=$ constante. Portanto a primeira equação do sistema (2.23) pode ser escrita como

$$
\tau_{1}\left(a^{2}+b^{2}\right)=0
$$

Sendo $a^{2}+b^{2} \neq 0$, concluímos que $\tau_{1}=0$ e, consequentemente, $\gamma$ é uma geodésica. 


\section{Capítulo 3}

\section{Curvas bi-harmônicas próprias em uma variedade Riemanniana}

Neste capítulo vamos descrever geometricamente a equação de Euler-Lagrange em uma variedade Riemanniana fazendo uso o referencial de Frenet (veja Definição 3.0.12), de acordo com [10]. Em seguida, consideraremos o caso de variedades Riemannianas bidimensionais (ver [11]) e, como primeiro resultado importante (ver Proposição 3.1.1), provaremos que ao longo de uma curva bi-harmônica não geodésica a curvatura de Gauss da superfície é constante, positiva e igual ao quadrado da curvatura geodésica.

Na Seção 3.2 focaremos nossa atenção ao caso das superfícies de revolução de $\mathbb{R}^{3}$. Serão, então, descritas curvas bi-harmônicas de algumas superfícies como o toro de revolução, as superfícies de Delaunay e o elipsóide de revolução e, também, daremos a solução explícita da equação bi-harmônica para o caso de superfícies de revolução com curvatura Gaussiana constante (ver Seção 3.2.2).

Precisamos da seguinte

Definição 3.0.12 (veja, por exemplo, [28]). O referencial de Frenet $\left\{F_{i}\right\}_{i=1, \ldots, n}$ associado à curva $\gamma: I \rightarrow\left(M^{n}, g\right)$, parametrizada pelo comprimento de arco, é a ortonormalização da $(n+1)-u p l a$

$$
\left\{\nabla_{\frac{\partial}{\partial t}}^{(k)} d \gamma\left(\frac{\partial}{\partial t}\right)\right\}_{k=0,1, \ldots, n}
$$


descrita por:

$$
\begin{aligned}
& F_{1}=d \gamma\left(\frac{\partial}{\partial t}\right), \\
& \nabla_{\frac{\partial}{\partial t}}^{\gamma} F_{1}=k_{1} F_{2}, \\
& \nabla_{\frac{\partial}{\partial t}}^{\gamma} F_{i}=-k_{i-1} F_{i-1}+k_{i} F_{i+1}, \quad \forall i=2, \ldots, n-1, \\
& \nabla_{\frac{\partial}{\partial t}}^{\gamma} F_{n}=-k_{n-1} F_{n-1},
\end{aligned}
$$

onde as funções $\left\{k_{1}, k_{2}, \ldots, k_{n-1}\right\}$ são chamadas de curvaturas de $\gamma$ e $\nabla^{\gamma}$ é a conexão no fibrado "pull-back" $\gamma^{-1}(T M)$, como na Seção 1.3. Note que $F_{1}=T=\gamma^{\prime}$ é o campo de vetores tangentes ao longo da curva $\gamma$.

Usando o referencial de Frenet, a equação bi-harmônica (1.46) se reduz a um sistema de equações diferenciais nas curvaturas de $\gamma$. Mais precisamente, temos o seguinte:

Proposição 3.0.13. Seja $\gamma: I \subset \mathbb{R} \rightarrow\left(M^{n}, g\right) \quad(n \geq 2)$ uma curva parametrizada pelo comprimento de arco, de um intervalo aberto de $\mathbb{R}$ em uma variedade Riemanniana $(M, g)$. Então, $\gamma$ é bi-harmônica se, e somente se,

$$
\left\{\begin{array}{l}
k_{1} k_{1}^{\prime}=0 \\
k_{1}^{\prime \prime}-k_{1}^{3}-k_{1} k_{2}^{2}+k_{1} \mathrm{R}\left(F_{1}, F_{2}, F_{1}, F_{2}\right)=0 \\
2 k_{1}^{\prime} k_{2}+k_{1} k_{2}^{\prime}+k_{1} \mathrm{R}\left(F_{1}, F_{2}, F_{1}, F_{3}\right)=0 \\
k_{1} k_{2} k_{3}+k_{1} \mathrm{R}\left(F_{1}, F_{2}, F_{1}, F_{4}\right)=0 \\
k_{1} \mathrm{R}\left(F_{1}, F_{2}, F_{1}, F_{j}\right)=0, \quad j=5, \ldots, n .
\end{array}\right.
$$

Demonstração. Por cálculo direto, temos que

$$
\nabla_{T}^{3} T=-3 k_{1}^{\prime} k_{1} F_{1}+\left(k_{1}^{\prime \prime}-k_{1}^{3}-k_{1} k_{2}^{2}\right) F_{2}+\left(2 k_{1}^{\prime} k_{2}+k_{1} k_{2}^{\prime}\right) F_{3}+k_{1} k_{2} k_{3} F_{4} .
$$

Escrevendo $\mathrm{R}\left(T, \nabla_{T} T\right) T$ no referencial de Frenet $\left\{F_{i}\right\}_{i=1, \ldots, n}$ e observando que $\mathrm{R}\left(F_{1}, F_{2}, F_{1}, F_{1}\right)=$ 0 , segue o resultado.

Como estamos interessados em curvas bi-harmônicas próprias (i.e. $k_{1} \neq 0$ ), o sistema anterior se torna

$$
\left\{\begin{array}{l}
k_{1}=\text { constante } \neq 0 \\
-k_{1}^{2}-k_{2}^{2}+\mathrm{R}\left(F_{1}, F_{2}, F_{1}, F_{2}\right)=0 \\
k_{2}^{\prime}+\mathrm{R}\left(F_{1}, F_{2}, F_{1}, F_{3}\right)=0 \\
k_{2} k_{3}+\mathrm{R}\left(F_{1}, F_{2}, F_{1}, F_{4}\right)=0 \\
\mathrm{R}\left(F_{1}, F_{2}, F_{1}, F_{j}\right)=0, \quad j=5, \ldots, n .
\end{array}\right.
$$


Observação 3.0.14. O sistema (2.10) segue diretamente de (3.2) ao observarmos que, no caso de $\mathbb{H}^{2}$, resulta $F_{i}=0$ se $i>2, k_{i}=0$ para $i>1$ e $\mathrm{R}\left(F_{1}, F_{2}, F_{1}, F_{2}\right)=1$.

\subsection{Estudo das curvas bi-harmônicas em uma superfície}

O objetivo desta seção é apresentar o artigo [11], que trata da geometria das curvas biharmônicas próprias de superfícies.

Sejam $\left(M^{2}, g\right)$ uma superfície Riemanniana e $\gamma: I \rightarrow\left(M^{2}, g\right)$ uma curva diferenciável, parametrizada pelo comprimento de arco. Vimos no Capítulo 1, que $\gamma$ é uma curva bi-harmônica se satisfizer a equação de Euler-Lagrange:

$$
\nabla_{\gamma^{\prime}}^{2} \tau+\mathrm{R}\left(\gamma^{\prime}, \tau_{\gamma}\right) \gamma^{\prime}=0
$$

sendo $\tau_{\gamma}=\nabla_{\gamma^{\prime}} \gamma^{\prime}$. Indicando por $\{T, N\}$ campos ortonormais tangentes a $M^{2}$ ao longo de $\gamma$, com $T=\gamma^{\prime}$ então, pela (3.1), valem as equações de Frenet:

$$
\nabla_{T} T=\kappa_{g} N \quad \text { e } \quad \nabla_{T} N=-\kappa_{g} T
$$

onde $\kappa_{g}=\left\|\nabla_{\gamma^{\prime}} \gamma^{\prime}\right\|$ é a curvatura geodésica de $\gamma$. Como observado anteriormente, estamos interessados em curvas bi-harmônicas próprias. Impondo esta condição e fazendo uso de (3.2) temos

$$
\left\{\begin{array}{l}
\kappa_{g}=\text { constante } \neq 0 \\
\kappa_{g}^{2}=K
\end{array}\right.
$$

onde $K=\mathrm{R}(T, N, T, N)$ é a curvatura Gaussiana da superfície.

Do sistema (3.5) segue o seguinte:

Proposição 3.1.1. Seja $\gamma: I \rightarrow\left(M^{2}, g\right)$ uma curva diferenciável em uma superfície $M^{2}$. Então, se $\gamma$ é uma curva bi-harmônica própria, a curvatura Gaussiana é constante ao longo de $\gamma$, positiva e igual ao quadrado da curvatura geodésica de $\gamma$. Portanto, se $M^{2}$ tiver curvatura Gaussiana não-positiva, toda curva bi-harmônica em $M^{2}$ é uma geodésica.

\subsection{Curvas bi-harmônicas em superfícies de revolução de $\mathbb{R}^{3}$}

Seja $\alpha(u)=(f(u), 0, g(u)), u \in I \subset \mathbb{R}$, uma curva diferenciável parametrizada pelo comprimento de arco com $f(u) \neq 0, \forall u \in I$. Considere a superfície obtida pela rotação desta curva 
em torno do eixo $z$, com a parametrização natural dada por:

$$
X(u, v)=(f(u) \cos v, f(u) \operatorname{sen} v, g(u))
$$

onde $v$ é o ângulo de rotação em torno do eixo $z$. Como

$$
\begin{array}{l|l}
E=\left\langle X_{u}, X_{u}\right\rangle=1, & e=\left\langle N, X_{u u}\right\rangle=f^{\prime}(u) g^{\prime \prime}(u)-g^{\prime}(u) f^{\prime \prime}(u), \\
F=\left\langle X_{u}, X_{v}\right\rangle=0, & f=\left\langle N, X_{u v}\right\rangle=0, \\
G=\left\langle X_{v}, X_{v}\right\rangle=f^{2}(u), & g=\left\langle N, X_{v v}\right\rangle=g^{\prime}(u) f(u), \\
N=\left(-g^{\prime}(u) \cos v, g^{\prime}(u) \operatorname{sen} v, f^{\prime}(u)\right), &
\end{array}
$$

a curvatura Gaussiana da superfície é dada por

$$
\mathrm{K}=-\frac{f^{\prime \prime}(u)}{f(u)}
$$

Logo K só depende da coordenada $u$, ou seja é constante ao longo de cada paralelo. Consequentemente, se a curvatura Gaussiana é constante ao longo de uma curva, ou a curva é um paralelo, ou está contida em uma parte da superfície com curvatura Gaussiana constante. Em particular, uma curva bi-harmônica própria ou é um paralelo ou está contida em uma superfície com curvatura Gaussiana constante. Por essa razão, estudaremos as curvas bi-harmônicas em superfícies de revolução com curvatura Gaussiana constante separadamente na Seção 3.2.2

Por hora, nos ocuparemos em determinar as superfícies de revolução nas quais todos os paralelos são curvas bi-harmônicas. Temos o seguinte:

Proposição 3.2.1. Seja $M$ uma superfície de revolução, localmente parametrizada por (3.6). Então um paralelo $u=\bar{u} \in I$ é uma curva bi-harmônica de $M$ se e somente se é ponto crítico da função $\eta(u):=f(u) f^{\prime}(u)$.

Demonstração. Seja $X(u, v)$ a parametrização de $M$ dada pela (3.6) e considere a parametrização pelo comprimento de arco do paralelo $u=\bar{u}$ dada por

$$
\gamma_{\bar{u}}(t)=\left(f(\bar{u}) \cos \left(\frac{t}{f(\bar{u})}\right), f(\bar{u}) \operatorname{sen}\left(\frac{t}{f(\bar{u})}\right), g(\bar{u})\right) .
$$

Calculemos sua curvatura geodésica $\kappa_{g}$. Indicando por $\sigma\left(\gamma_{\bar{u}}, X_{u}\right)$ o ângulo entre $\gamma_{\bar{u}}$ e $X_{u}$, vale a fórmula (ver, por exemplo, [6]):

$$
\kappa_{g}=\sigma^{\prime}\left(\gamma, X_{u}\right)+\frac{-\frac{F}{E}\left(E_{u} u^{\prime}+E_{v} v^{\prime}\right)-E_{v} u^{\prime}+G_{u} v^{\prime}+2 F_{u} u^{\prime}}{2 \sqrt{E G-F^{2}}} .
$$


Usando (3.7) e o fato que

$$
\cos \sigma=\left\langle\gamma_{\bar{u}}^{\prime}, X_{u}\right\rangle \equiv 0
$$

resulta que

$$
\kappa_{g}=\frac{f^{\prime}(\bar{u})}{f(\bar{u})}
$$

Consequentemente, substituindo (3.8) e (3.9) na segunda equação do sistema (3.5), resulta que $\gamma_{\bar{u}}$ é bi-harmônica se e somente se

$$
f^{\prime 2}(\bar{u})+f^{\prime \prime}(\bar{u}) f(\bar{u})=\eta^{\prime}(\bar{u})=0 .
$$

Teorema 3.2.2. Seja $M^{2} \subset \mathbb{R}^{3}$ uma superfície de revolução obtida pela rotação, ao redor do eixo $z$, da curva (parametrizada pelo comprimento de arco) $\alpha(u)=(f(u), 0, g(u)), u \in I \subset \mathbb{R}$, com $f(u) \neq 0$. Então, todos os paralelos de $M$ são curvas bi-harmônicas se e só se vale um dos casos seguintes:

1. $f$ é constante e $M$ é um cilindro circular reto;

$o u$

2. $f(u)= \pm c \sqrt{u}$ $g(u)=u \sqrt{\frac{4 u-c^{2}}{4 u}}-\frac{c^{2}}{8} \log \left(8 u+8 u \sqrt{\frac{4 u-c^{2}}{4 u}}-c^{2}\right)+c_{1}$, onde c e $c_{1}$ são constantes positivas (veja Figura 3.1).

Demonstração. Da Proposição 3.2.1 segue que todos os paralelos de $M$ são bi-harmônicos se e somente se $f(u)$ satisfizer a seguinte equação diferencial de segunda ordem:

$$
f^{\prime 2}(u)+f^{\prime \prime}(u) f(u)=0 .
$$

É evidente que $f=$ constante satisfaz (3.11). Neste caso todos os paralelos serão geodésicas (já que $k_{g}=0$ ), e a superfície de revolução será um cilindro circular reto. Assumindo que $f$ não seja constante e integrando a equação (3.11) obtemos que $f(u)= \pm c \sqrt{u+d}, c \in \mathbb{R}, c>0$. A menos de uma translação da curva, podemos considerar $d=0$. Como $\alpha$ está parametrizada pelo 
comprimento de arco, $f^{\prime 2}+g^{\prime 2}=1$, logo

$$
\begin{aligned}
g(u) & = \pm \int \sqrt{\frac{4 u-c^{2}}{4 u}} d u= \pm\left(u \sqrt{\frac{4 u-c^{2}}{4 u}}-\frac{c^{2}}{8} \int \frac{1}{u} \sqrt{\frac{4 u}{4 u-c^{2}}} d u\right) \\
& = \pm\left(u \sqrt{\frac{4 u-c^{2}}{4 u}}-\frac{c^{2}}{8} \log \left(8 u+8 u \sqrt{\frac{4 u-c^{2}}{4 u}}-c^{2}\right)\right)+c_{1}, \quad c_{1} \in \mathbb{R}
\end{aligned}
$$

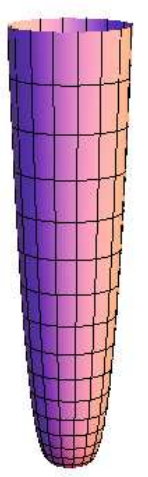

Figura 3.1: Superfície de revolução cujos paralelos são todos bi-harmônicos.

\subsubsection{Exemplos de curvas bi-harmônicas em uma superfície de revolução com curvatura Gaussiana não constante}

Vejamos alguns exemplos.

Exemplo 3.2.3 (O toro de revolução). Considere a parametrização do toro de revolução

$$
X(u, v)=((a+r \cos (u / r)) \cos v,(a+r \cos (u / r)) \operatorname{sen} v, r \operatorname{sen}(u / r)),
$$

onde $a>r>0$. Sua curvatura Gaussiana é

$$
K=-\frac{f^{\prime \prime}(u)}{f(u)}=\frac{\cos (u / r)}{r(a+\cos (u / r))} .
$$

Pela Proposição 3.2.1, um paralelo do toro é uma curva bi-harmônica se

$$
\operatorname{sen}^{2}(u / r)-\cos (u / r) \frac{(a+r \cos (u / r)}{r}=0,
$$


ou seja,

$$
u=r \arccos \left(\frac{-a \pm \sqrt{a^{2}+8 r^{2}}}{4 r}\right)
$$

Logo, os paralelos bi-harmônicos do toro são:

$$
u_{1}=r \arccos \left(\frac{-a+\sqrt{a^{2}+8 r^{2}}}{4 r}\right), \quad u_{2}=2 \pi r-u_{1} .
$$

A Figura 3.2 mostra os paralelos bi-harmônicos do toro no caso em que $a=3$ e $r=1$.

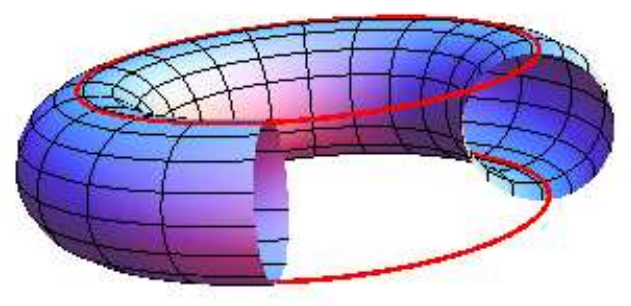

Figura 3.2: Paralelos bi-harmônicos do toro com $a=3$ e $r=1$.

Exemplo 3.2.4 (Superfícies de Delaunay). Devido à um teorema de C. Delaunay (veja [18]) sabemos que as superfícies de revolução imersas em $\mathbb{R}^{3}$, com curvatura média constante, são aquelas obtidas pela rotação de roulettes de cônicas ao redor de seu eixo. Por essa razão, superfícies de revolução com curvatura média constante são chamadas de superfícies de Delaunay.

Usaremos a parametrização das superfícies de Delaunay dada por M.P. do Carmo e M. Dajczer em [20]. Primeiro definimos

$$
\left\{\begin{array}{l}
\rho(u)=\frac{\sqrt{1+B^{2}+2 B \operatorname{sen}(2 H u)}}{2 H} \\
\lambda(u)=\int_{0}^{u} f(s) d s
\end{array}\right.
$$

onde $B$ é uma constante não negativa, $H$ é a curvatura média da superfície $M$ e

$$
f(s)=\frac{1+B \operatorname{sen}(2 H s)}{\sqrt{1+B^{2}+2 B \operatorname{sen}(2 H s)}} .
$$

Uma parametrização das superfícies de Delaunay é obtida pela rotação ao redor do eixo z da curva, parametrizada por comprimento de arco, $\alpha(u)=(\rho(u), 0, \lambda(u))$, ou seja

$$
X(u, v)=(\rho(u) \cos v, \rho(u) \operatorname{sen} v, \lambda(u))
$$

Dependendo do valor de B, obtemos quatro tipos de superfícies de Delaunay, estas são: 
1. O cilindro circular reto $(B=0)$;

2. Os ondulóides $(0<B<1)$;

3. A esfera $(B=1)$;

4. Os nodóides $(B>1)$.

Da Proposição (3.2.1) segue que paralelos de superfícies de Delaunay que são curvas biharmônicas são dados por $u=$ constante tal que:

$$
\frac{\rho^{\prime}(u)^{2}}{\rho(u)^{2}}+\frac{\rho^{\prime \prime}(u)}{\rho(u)}=0
$$

Como

$$
\rho^{\prime}(u)=\frac{B \cos (2 H u)}{\sqrt{1+B^{2}+2 B \operatorname{sen}(2 H u)}}
$$

e

$$
\rho^{\prime \prime}(u)=-\frac{2 H B \operatorname{sen}(2 H u)}{\sqrt{1+B^{2}+2 B \operatorname{sen}(2 H u)}},
$$

teremos

$$
-\frac{4 H B^{2} \operatorname{sen}(2 H u)}{\left(1+B^{2}+2 B \operatorname{sen}(2 H u)\right)}=0
$$

Portanto, $u$ deve ser da forma $u=k \pi / 2 H, k \in \mathbb{Z}$. Observe-se que o valor de $u$ não depende de $B$, mas apenas da curvatura média $H$ da superfície $M$.
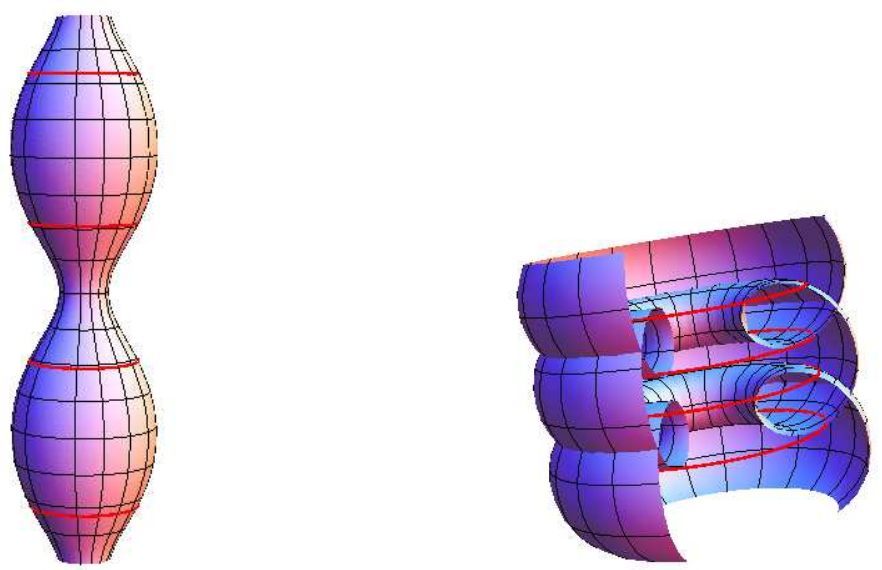

Figura 3.3: Paralelos bi-harmônicos no ondulóide e no nodóide, respectivamente. 
É importante ressaltar que, em geral, é complicado parametrizar a curva geratriz da superfície de revolução pelo comprimento de arco. Algumas vezes é mais fácil calcular explicitamente a curvatura geodésica da curva e, então, utilizar a equação $\mathrm{K}=\kappa_{g}^{2}$. Isto é ilustrado no exemplo que segue.

Exemplo 3.2.5 (O elipsóide de revolução). Seja $M^{2} \subset \mathbb{R}^{3}$ o elipsóide de revolução parametrizado por

$$
X(u, v)=(a \cos u \cos v, a \cos u \operatorname{sen} v, c \operatorname{sen} u)
$$

onde $a, c \in \mathbb{R}^{+}$. Os coeficientes da primeira e segunda formas fundamentais de $M$ são

$$
\begin{array}{l|l}
E=\left\langle X_{u}, X_{u}\right\rangle=a^{2} \operatorname{sen}^{2} u+c^{2} \cos ^{2} u, & \begin{array}{l}
e=\left\langle N, X_{u u}\right\rangle=\frac{c a}{\sqrt{c^{2} \cos ^{2} u+a^{2} \operatorname{sen}^{2} u}} \\
F=\left\langle X_{u}, X_{v}\right\rangle=0,
\end{array} \\
G=\left\langle X_{v}, X_{v}\right\rangle=a^{2} \cos ^{2} u & g=\left\langle N, X_{u v}\right\rangle=0, \\
g=\left\langle N, X_{v v}\right\rangle=\frac{c a \cos ^{2} u}{\sqrt{c^{2} \cos ^{2} u+a^{2} \operatorname{sen}^{2} u}},
\end{array}
$$

onde

$$
N=\frac{(-c \cos u \cos v,-c \cos u \operatorname{sen} v,-a \operatorname{sen} u)}{\sqrt{c^{2} \cos ^{2} u+a^{2} \operatorname{sen}^{2} u}} .
$$

Logo, sua curvatura Gaussiana é:

$$
K=\frac{c^{2}}{\left(a^{2} \operatorname{sen}^{2} u+c^{2} \cos ^{2} u\right)^{2}} .
$$

A curvatura geodésica de um paralelo de $M$ parametrizado pelo comprimento de arco é dada por:

$$
\begin{aligned}
\kappa_{g} & =\sigma^{\prime}\left(\gamma_{u}, X_{u}\right)+\frac{-\frac{F}{E}\left(E_{u} u^{\prime}+E_{v} v^{\prime}\right)-E_{v} u^{\prime}+G_{u} v^{\prime}+2 F_{u} u^{\prime}}{2 \sqrt{E G-F^{2}}} \\
& =\frac{\operatorname{sen} u}{\cos u \sqrt{a^{2} \operatorname{sen}^{2} u+c^{2} \cos ^{2} u}} .
\end{aligned}
$$

Logo, da equação $\mathrm{K}=\kappa_{g}^{2}$ resulta que

$$
\frac{c^{2}}{\left(a^{2} \operatorname{sen}^{2} u+c^{2} \cos ^{2} u\right)^{2}}=\frac{\operatorname{sen}^{2} u}{\cos ^{2} u\left(a^{2} \operatorname{sen}^{2} u+c^{2} \cos ^{2} u\right)},
$$

ou seja,

$$
\left(a^{2}-c^{2}\right) \operatorname{sen}^{4} u+2 c^{2} \operatorname{sen}^{2} u-c^{2}=0 .
$$

Portanto, se $M^{2}$ não for uma esfera, i.e. para $a \neq c$, chegamos à equação

$$
\left(\operatorname{sen}^{2} u+\frac{c^{2}}{\left(a^{2}-c^{2}\right)^{2}}\right)^{2}-\frac{c^{4}}{\left(a^{2}-c^{2}\right)^{2}}-\frac{c^{2}}{a^{2}-c^{2}}=0,
$$


cujas soluções são

$$
\operatorname{sen}^{2} u=\left\{\begin{array}{l}
\frac{c}{c+a}, \\
\frac{c}{c-a}
\end{array}\right.
$$

A segunda equação nunca é satisfeita; já a primeira nos dá os dois paralelos bi-harmônicos $u=\operatorname{arcsen}( \pm \sqrt{c /(c+a)})$.

\subsubsection{Curvas bi-harmônicas em superfícies de revolução com curvatura Gaus- siana constante}

Seja $M^{2} \subset \mathbb{R}^{3}$ uma superfície de revolução com curvatura Gaussiana constante positiva $\mathrm{K}=\frac{1}{a^{2}}$. A superfície $M^{2}$ pode ser parametrizada por (veja, por exemplo, [6]):

$$
X(u, v)=(f(u) \cos v, f(u) \operatorname{sen} v, g(u))
$$

onde

$$
f(u)=b \cos (u / a) \quad e \quad g(u)=\int_{0}^{u} \sqrt{1-\frac{b^{2}}{a^{2}} \operatorname{sen}^{2}\left(\frac{s}{a}\right)} d s, \quad b \in \mathbb{R}^{+}
$$

Seja $\gamma(t)=X(u(t), v(t))$ uma curva em $M^{2}$ parametrizada por comprimento de arco. Calcularemos agora o triedro de Darboux (ver [6]) ao longo de $\gamma$. Como

$$
X_{u}=\left(\frac{d f}{d u} \cos v, \frac{d f}{d u} \operatorname{sen} v, \frac{d g}{d u}\right) \quad \text { e } \quad X_{v}=(-f(u) \operatorname{sen} v, f(u) \cos v, 0),
$$

o vetor unitário normal a $M$ restrito a $\gamma$ é dado por

$$
N(t):=\frac{X_{u} \wedge X_{v}}{\left\|X_{u} \wedge X_{v}\right\|_{\mid \gamma(t)}}=\left(\frac{d g}{d u} \cos v, \frac{d g}{d u} \operatorname{sen} v,-\frac{d f}{d u}\right)_{\mid \gamma(t)} .
$$

Sendo $\gamma$ parametrizada pelo comprimento de arco, o campo de vetores

$$
\gamma^{\prime}(t)=u^{\prime} X_{u}+v^{\prime} X_{v}
$$

satisfaz

$$
1=\left\|\gamma^{\prime}(t)\right\|^{2}=u^{\prime 2}+v^{\prime 2} f^{2}(u) .
$$

O triedro de Darboux ao longo de $\gamma$ será, então, dado por:

$$
\begin{aligned}
& T=\gamma^{\prime}(t)=u^{\prime} X_{u}+v^{\prime} X_{v}, \\
& V=N \wedge T=v^{\prime} f X_{u}-\frac{u^{\prime}}{f} X_{v}, \\
& N=\left(\frac{d g}{d u} \cos v, \frac{d g}{d u} \operatorname{sen} v,-\frac{d f}{d u}\right) .
\end{aligned}
$$


Das equações de Darboux:

$$
\begin{aligned}
& T^{\prime}=\kappa_{g} V+\kappa_{n} N, \\
& V^{\prime}=-\kappa_{g} T+\tau_{g} N, \\
& N^{\prime}=-\kappa_{n} T-\tau_{g} V,
\end{aligned}
$$

onde $\kappa_{n}$ é a curvatura normal e $\tau_{g}$ é a torsão geodésica de $\gamma$, segue que

$$
\left\langle T^{\prime}, V\right\rangle=\kappa_{g}
$$

Usando (3.13), (3.15) e $u^{\prime 2}+v^{\prime 2} f^{2}=1$, teremos

$$
\left\langle T^{\prime}, V\right\rangle=-v^{\prime \prime} u^{\prime} f+u^{\prime \prime} v^{\prime} f-v^{\prime}\left(u^{\prime}\right)^{2} \frac{d f}{d u}-v^{\prime} \frac{d f}{d u} .
$$

Agora, derivando $u^{\prime 2}+f^{2} v^{\prime 2}=1$ e usando o fato de $\gamma$ não ser um paralelo (i.e. $u^{\prime} \neq 0$ ), obtemos

$$
u^{\prime \prime}=-\frac{v^{\prime} v^{\prime \prime} f^{2}+v^{\prime 2} u^{\prime} f \frac{d f}{d u}}{u^{\prime}} .
$$

Substituindo em (3.16) resulta que

$$
\kappa_{g}=-\frac{v^{\prime \prime} f+2 v^{\prime} u^{\prime} \frac{d f}{d u}}{u^{\prime}}
$$

Dessa forma, a equação bi-harmônicas $\kappa_{g}^{2}=K$ se torna

$$
-\frac{v^{\prime \prime} f+2 u^{\prime} v^{\prime} f^{\prime}}{u^{\prime}}=\frac{1}{a}
$$

Multiplicando os dois lados por $f u^{\prime}$, temos

$$
-\frac{d}{d t} v^{\prime}(t) f^{2}(u(t))=\frac{1}{a} f(u) u^{\prime}(t)
$$

Logo, se substituirmos $f(u)=b \cos (u / a)$, teremos

$$
\frac{d}{d t}\left(v^{\prime} b^{2} \cos ^{2}(u / a)\right)=\frac{d}{d t}(-b \operatorname{sen}(u / a))
$$

Dessa forma, a condição para que $\gamma(t)=X(u(t), v(t))$ seja uma curva bi-harmônica parametrizada por comprimento de arco é dada pelo sistema

$$
\left\{\begin{array}{l}
v^{\prime} b^{2} \cos ^{2}(u / a)=-b \operatorname{sen}(u / a)+c \\
v^{\prime 2} b^{2} \cos ^{2}(u / a)+u^{\prime 2}=1
\end{array}\right.
$$

Resolvendo esse sistema com respeito a $u^{\prime}$ obtemos

$$
u^{\prime}(t)= \pm \frac{1}{b \cos (u / a)} \sqrt{b^{2}-c^{2}-2 b^{2} \operatorname{sen}^{2}(u / a)+2 b c \operatorname{sen}(u / a)}
$$


portanto

$$
\pm \frac{a}{\sqrt{2}} \operatorname{arcsen}\left(\frac{c-2 b \operatorname{sen}(u / a)}{\sqrt{2 b^{2}-c^{2}}}\right)=t+A, \quad A \in \mathbb{R}
$$

com $2 b^{2}-c^{2}>0$. Explicitando $u(t)$, resulta

$$
u(t)=a \operatorname{arcsen}\left(\frac{c \pm \sqrt{2 b^{2}-c^{2}} \operatorname{sen}(B+(\sqrt{2} / a) t)}{2 b}\right), \quad B:=\frac{\sqrt{2}}{a} A .
$$

Quanto a $v(t)$, do sistema (3.17) resulta que

$$
v^{\prime}(t)=-\frac{b \operatorname{sen}(u(t) / a)+c}{b^{2} \cos ^{2}(u(t) / a)}
$$

$\log 0$

$$
v(t)=\int_{0}^{t}\left(-\frac{\operatorname{sen}(u(s) / a)}{b \cos ^{2}(u(s) / a)}+\frac{c}{b^{2} \cos ^{2}(u(s) / a)}\right) d s .
$$

Desta forma, encontramos a solução geral para as curvas bi-harmônicas em superfícies de revolução com curvatura Gaussiana constante positiva que não são paralelos. A Figura 3.4 mostra essas curvas nos três diferentes tipos de superfícies de revolução com curvatura Gaussiana constante positiva:

1. O tipo esfera $(a=b)$;

2. O tipo bola de futebol americano $(b<a)$;

3. O tipo barril $(a<b)$.
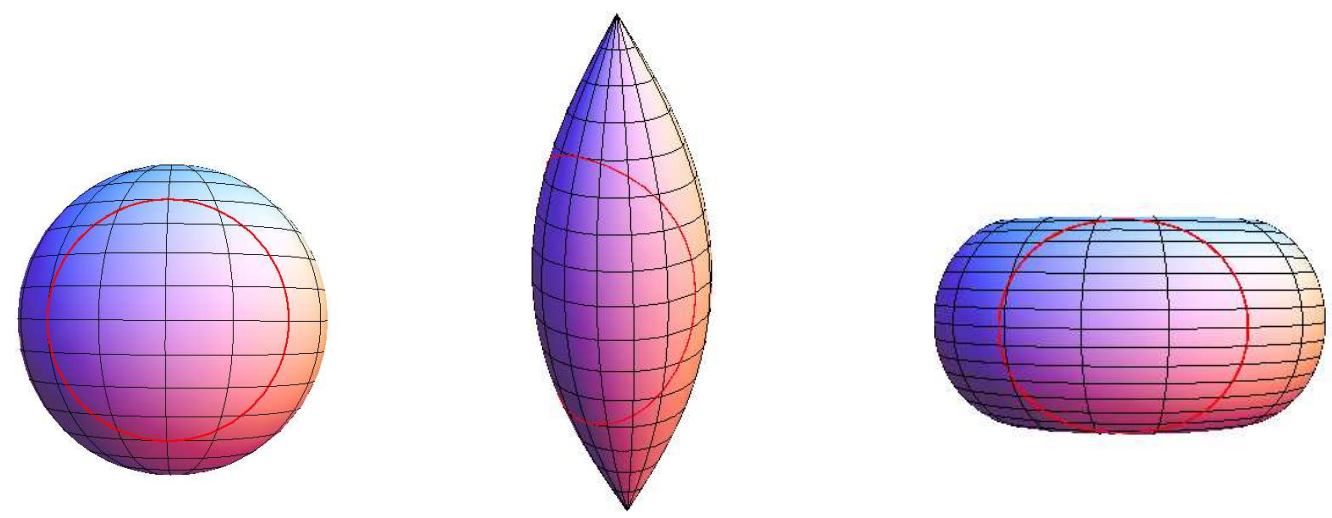

Figura 3.4: Curvas bi-harmônicas em superfícies com curvatura Gaussiana constantes positiva. 


\section{Capítulo 4}

\section{Classificação das curvas \\ bi-harmônicas dos BCV-espaços}

Neste capítulo daremos a classificação completa das curvas bi-harmônicas nas 3-variedades de Bianchi-Cartan-Vranceanu utilizando as condições obtidas no Capítulo 2. Cabe aqui ressaltar que no caso de variedades Riemannianas tridimensionais com curvatura seccional constante $K \leq 0$, as curvas bi-harmônicas são geodésicas (veja [19] para $K=0$, e [8] para $K<0$ ). Além disso, na Seção 4.5 provaremos que no caso em que K > 0 as curvas bi-harmônicas são hélices, ou seja curvas que possuem curvatura geodésica e torsão geodésica constantes.

\subsection{Espaços de Bianchi-Cartan-Vranceanu e sua estrutura Rie- manniana}

É um resultado clássico da geometria diferencial (veja, por exemplo, [35]) que a dimensão máxima do grupo das isometrias de uma variedade Riemanniana tridimensional é 6 e, nesse caso, a variedade é uma forma espacial. Também não existem 3-variedades com grupo de isometrias de dimensão 5 (veja [23]). Além disso, se uma 3-variedade possui grupo de isometrias 4-dimensional, então é uma variedade Riemanniana homogênea.

Para as variedades Riemannianas homogêneas tridimensionais existe uma representação local dada pela seguinte família a dois parâmetros de métricas Riemannianas:

$$
g_{\ell, m}=\frac{d x^{2}+d y^{2}}{\left[1+m\left(x^{2}+y^{2}\right)\right]^{2}}+\left(d z+\frac{\ell}{2} \frac{y d x-x d y}{\left[1+m\left(x^{2}+y^{2}\right)\right]}\right)^{2}, \quad \ell, m \in \mathbb{R},
$$


definidas em $M=\mathbb{R}^{3}$ se $m \geq 0$, se não em $M=\left\{(x, y, z) \in \mathbb{R}^{3}: x^{2}+y^{2}<-1 / m\right\}$.

As métricas $g_{\ell, m}$, denominadas métricas de Bianchi-Cartan-Vranceanu, podem ser encontradas na classificação das variedades Riemannianas homogêneas tridimensionais que foi dada por L. Bianchi em 1897 (veja [3]) e, mais tarde, aparecem na forma (4.1) em [14, 35], com É. Cartan e G. Vranceanu (respectivamente).

A importância e o interesse na geometria para esta família de métricas está no fato dela incluir todas as métricas homogêneas tridimensionais cujo grupo de isometrias é de dimensão 4 ou 6 , exceto às de curvatura seccional constante negativa. Indicando com $\mathbb{H}^{2}$ o disco hiperbólico de raio $1 /(2 \sqrt{-m})$ e com $\mathbb{S}^{2}$ a esfera de $\mathbb{R}^{3}$ de raio $1 /(2 \sqrt{m})$, temos a situação ilustrada na Figura 4.1 .

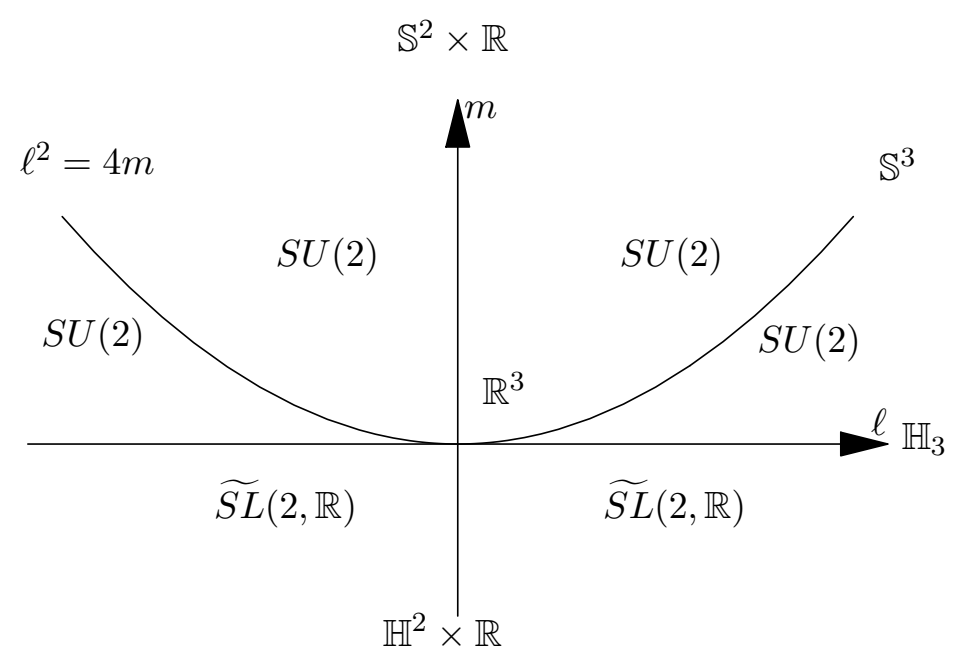

Figura 4.1: Descrição geométrica da métrica $g_{\ell, m}$ (veja [32]).

Observe que:

- se $\ell^{2}=4 m$, então $M$ possui curvatura seccional constante e não negativa, os seja $M$ é o espaço Euclidiano $\mathbb{R}^{3}$ ou a esfera $\mathbb{S}^{3}$;

- se $\ell=0$, então $M$ é produto de uma superfície de curvatura Gaussiana constante $4 m$ com a reta real $\mathbb{R}$

- se $\ell \neq 0$ e $m>0, M$ é localmente o grupo unitário especial $S U(2)$;

- se $\ell \neq 0$ e $m<0, M$ é localmente o recobrimento universal do grupo linear especial, i.e. $\widetilde{S L}(2, \mathbb{R})$; 
- se $\ell \neq 0$ e $m=0$, obtemos o espaço de Heisenberg $\mathbb{H}_{3}$ munido de uma métrica invariante à esquerda.

Para que possamos fazer o estudo das curvas bi-harmônicas nesses espaços, vamos estudar sua estrutura Riemanniana. A métrica de Bianchi-Cartan-Vranceanu pode ser escrita através de 1-formas, como

$$
g_{\ell, m}=\sum_{i=1}^{3} w_{i} \otimes w_{i}
$$

onde

$$
w_{1}=\frac{d x}{F} ; \quad w_{2}=\frac{d y}{F} ; \quad w_{3}=d z+\frac{\ell}{2} \frac{y d x-x d y}{F}, \quad F=\left[1+m\left(x^{2}+y^{2}\right)\right]
$$

A base ortonormal de campos de vetores duais às 1-formas é

$$
E_{1}=F \frac{\partial}{\partial x}-\frac{\ell y}{2} \frac{\partial}{\partial z} ; \quad E_{2}=F \frac{\partial}{\partial x}+\frac{\ell x}{2} \frac{\partial}{\partial z} ; \quad E_{3}=\frac{\partial}{\partial z} .
$$

Vamos agora dar a expressão, com respeito à base ortonormal (4.3), da conexão Levi-Civita e das componentes não nulas do tensor curvatura. Cabe aqui observar que adotaremos as seguintes notações e convenção de sinal. O operador de curvatura é dado por:

$$
\mathrm{R}(X, Y) Z=\nabla_{Y} \nabla_{X}-\nabla_{X} \nabla_{Y}+\nabla_{[X, Y]} Z
$$

onde $X, Y, Z$ são campos de vetores diferenciáveis em $M$. Além disso,

$$
\mathrm{R}_{i j k}=\mathrm{R}\left(E_{i}, E_{j}\right) E_{k} \quad \mathrm{R}_{h i j k}=\mathrm{R}\left(E_{h}, E_{i}, E_{j}, E_{k}\right) .
$$

Temos que

$$
\begin{array}{l|l|l}
\nabla_{E_{1}} E_{1}=2 m y E_{2}, & \nabla_{E_{1}} E_{2}=-2 m y E_{1}+\frac{\ell}{2} E_{3}, & \nabla_{E_{3}} E_{1}=\nabla_{E_{1}} E_{3}=-\frac{\ell}{2} E_{2}, \\
\nabla_{E_{2}} E_{2}=2 m x E_{1}, \\
\nabla_{E_{3}} E_{3}=0
\end{array} \mid \begin{aligned}
& \nabla_{E_{2}} E_{1}=-2 m x E_{2}-\frac{\ell}{2} E_{3}, \\
& \nabla_{E_{3}} E_{2}=\nabla_{E_{2}} E_{3}=\frac{\ell}{2} E_{1},
\end{aligned}
$$

e

$$
R_{1212}=4 m-\frac{3}{4} \ell^{2} ; \quad R_{1313}=\frac{\ell^{2}}{4} \quad R_{2323}=\frac{\ell^{2}}{4} .
$$

\subsection{Condições para curvas bi-harmônicas em $\left(M, g_{\ell, m}\right)$}

Seja $\gamma: I \rightarrow\left(M, g_{\ell, m}\right)$ uma curva diferenciável parametrizada por comprimento de arco e seja

$$
F_{1}=T=\sum_{i=1}^{3} T_{i} E_{i}, \quad F_{2}=N=\sum_{i=1}^{3} N_{i} E_{i}, \quad F_{3}=B=\sum_{i=1}^{3} B_{i} E_{i}
$$


o referencial de Frenet tangente a $M$ ao longo de $\gamma$, como em (3.1), escrito na base (4.3). Então

(3.3) dá origem ao sistema

$$
\left\{\begin{array}{l}
k_{1}=\text { constante } \neq 0 \\
-k_{1}^{2}-k_{2}^{2}+R(T, N, T, N)=0 \\
k_{2}^{\prime}+R(T, N, T, B)=0 .
\end{array}\right.
$$

O próximo passo será determinar as expressões de $R(T, N, T, N)$ e $R(T, N, T, B)$. Temos que

$$
\begin{aligned}
R(T, N, T, N) & =R\left(\sum_{i=1}^{3} T_{i} E_{i}, \sum_{j=1}^{3} N_{j} E_{j}, \sum_{k=1}^{3} T_{k} E_{k}, \sum_{l=1}^{3} N_{l} E_{l}\right) \\
& =\sum_{i, j, k, l=1}^{3} T_{i} N_{j} T_{k} N_{l} R_{i j k l} \\
& =\left(T_{1} N_{2}-T_{2} N_{1}\right)^{2} R_{1212}+\left(T_{1} N_{3}-T_{3} N_{1}\right)^{2} R_{1313}+\left(T_{2} N_{3}-T_{3} N_{2}\right)^{2} R_{2323} \\
& =B_{3}^{2} R_{1212}+B_{2}^{2} R_{1313}+B_{1}^{2} R_{2323} \\
& =B_{3}^{2}\left(4 m-\frac{3}{4} \ell^{2}\right)+\left(B_{2}^{2}+B_{1}^{2}\right) \frac{\ell^{2}}{4} \\
& =\frac{\ell^{2}}{4}-\left(\ell^{2}-4 m\right) B_{3}^{2},
\end{aligned}
$$

e, também,

$$
\begin{aligned}
R(T, N, T, B) & =R\left(\sum_{i=1}^{3} T_{i} E_{i}, \sum_{j=1}^{3} N_{j} E_{j}, \sum_{k=1}^{3} T_{k} E_{k}, \sum_{l=1}^{3} B_{l} E_{l}\right) \\
& =\sum_{i, j, k, l=1}^{3} T_{i} N_{j} T_{k} B_{l} R_{i j k l} \\
& =\left(T_{1} N_{2}-T_{2} N_{1}\right)\left(T_{1} B_{2}-T_{2} B_{1}\right)\left(4 m-\frac{3}{4} \ell^{2}\right) \\
& +\left(T_{1} N_{3} T_{1}-T_{3} N_{1} T_{1}+T_{2} N_{3} T_{2}-T_{3} N_{2} T_{2}\right) B_{3} \frac{\ell^{2}}{4} \\
& +\left[\left(T_{3} N_{1} T_{3}-T_{1} N_{3} T_{3}\right)\left(T_{2} N_{3}-T_{3} N_{2}\right)\right. \\
& \left.+\left(T_{3} N_{2} T_{3}-T_{2} N_{3} T_{3}\right)\left(T_{3} N_{1}-T_{1} N_{3}\right)\right] \frac{\ell^{2}}{4} \\
& =\left(\ell^{2}-4 m\right) N_{3} B_{3} .
\end{aligned}
$$

Então, fazendo $k_{1}=\kappa_{g}$ (curvatura geodésica da curva) e $k_{2}=-\tau_{g}$ (torção da curva), o sistema (4.6) é reescrito na seguinte forma: 


$$
\left\{\begin{array}{l}
\kappa_{g}=\text { constante } \neq 0 \\
\kappa_{g}^{2}+\tau_{g}^{2}=\frac{\ell^{2}}{4}-\left(\ell^{2}-4 m\right) B_{3}^{2} \\
\tau_{g}^{\prime}=\left(\ell^{2}-4 m\right) N_{3} B_{3} .
\end{array}\right.
$$

A partir de agora vamos impor condições sobre os parâmetros $\ell$ e $m$, a fim de obter as equações explícitas das curvas bi-harmônicas nas variedades $\left(M, g_{\ell, m}\right)$. No caso $\ell=m=0$ (espaço euclidiano tridimensional) do sistema acima resulta que as curvas bi-harmônica são as geodésicas. Os demais casos são estudados nas seções que seguem.

\subsection{Equações explícitas das curvas bi-harmônicas em $\left(M, g_{\ell, m}\right)$ $\operatorname{com} \ell^{2} \neq 4 m$ e $m \neq 0$}

Nesse parágrafo faremos o estudo das curvas bi-harmônicas nos espaços de Bianchi-CartanVranceanu para o caso em que $\ell^{2} \neq 4 m$ e $m \neq 0$. Com isto estaremos considerando os casos dos espaços $\mathbb{S}^{2} \times \mathbb{R}, \mathbb{H}^{2} \times \mathbb{R}, S U(2)$ e $\widetilde{S L}(2, \mathbb{R})$.

Temos o seguinte resultado:

Teorema 4.3.1. Se $\gamma: I \rightarrow\left(M, g_{\ell, m}\right)$ é uma curva bi-harmônica própria parametrizada pelo comprimento de arco, então ela é uma hélice.

Demonstração. Seja $\gamma: I \rightarrow\left(M, g_{\ell, m}\right)$ uma curva parametrizada pelo comprimento de arco que não é geodésica. Então, das equações de Frenet resulta que

$$
\left\langle\nabla_{T} B, E_{3}\right\rangle=\left\langle\tau_{g} N, E_{3}\right\rangle=\tau_{g} N_{3}
$$

Por outro lado, usando as propriedades da conexão, temos

$$
\nabla_{T} B=\sum_{i} B_{i}^{\prime} E_{i}+\sum_{i, j} T_{j} B_{i} \nabla_{E_{j}} E_{i}
$$

de onde segue

$$
\left\langle\nabla_{T} B, E_{3}\right\rangle=B_{3}^{\prime}+\left(T_{1} B_{2}-T_{2} B_{1}\right) \frac{\ell}{2}
$$

e, então,

$$
\left\langle\nabla_{T} B, E_{3}\right\rangle=B_{3}^{\prime}-N_{3} \frac{\ell}{2}
$$


Comparando as equações (4.8) e (4.9) obtemos

$$
\tau_{g} N_{3}=B_{3}^{\prime}-\frac{\ell}{2} N_{3}
$$

Assumimos agora que $\gamma$ seja uma curva bi-harmônica própria. Primeiro mostraremos que $B_{3} \neq 0$. Para isto supomos que $B_{3}=0$, então (4.10) nos dá

$$
N_{3}\left(\tau_{g}+\frac{\ell}{2}\right)=0
$$

A última equação gera duas possibilidades:

1. se $N_{3}=0$ (e $\left.B_{3}=0\right)$ então $T= \pm E_{3}$ e $\gamma$ é uma geodésica, já que $\nabla_{E_{3}} E_{3}=0$.

2. Se $\left(\tau_{g}+\frac{\ell}{2}\right)=0$ então, usando a segunda equação do sistema (4.7), temos

$$
\kappa_{g}^{2}+\tau_{g}^{2}=\frac{\ell^{2}}{4}
$$

Logo $\kappa_{g}=0$ e, novamente, concluímos que $\gamma$ é uma geodésica.

Agora sabendo que $B_{3} \neq 0$, derivando a segunda equação de (4.7), fazendo uso da expressão de $\tau_{g}^{\prime}$ dada em (4.7) e do fato que $\ell^{2} \neq 4 m$, obtemos

$$
\tau_{g} N_{3}=-B_{3}^{\prime}
$$

Somando a (4.10) a esta última equação obtemos

$$
\left(4 \tau_{g}+\ell\right) N_{3}=0
$$

Nesse ponto, lançando mão da terceira equação de (4.7), concluímos que $\tau_{g}$ é constante, portanto $\gamma$ é uma hélice.

Corolário 4.3.2. Seja $\gamma: I \rightarrow\left(M, g_{\ell, m}\right)$ uma curva parametrizada pelo comprimento de arco. Então $\gamma$ é uma curva bi-harmônica própria se e somente se

$$
\left\{\begin{array}{l}
\kappa_{g}=\text { constante } \neq 0 \\
\tau_{g}=\text { constante } \\
N_{3}=0 \\
\kappa_{g}^{2}+\tau_{g}^{2}=\frac{\ell^{2}}{4}-\left(\ell^{2}-4 m\right) B_{3}^{2} .
\end{array}\right.
$$


Demonstração. Segue do sistema (4.7) utilizando $N_{3}=0$, obtido no teorema anterior.

Provaremos agora um resultado que nos permite obter as equações explícitas das curvas bi-harmônicas próprias, nos espaços que estamos considerando.

Lema 4.3.3. Seja $\gamma: I \rightarrow\left(M, g_{\ell, m}\right)$ uma curva parametrizada pelo comprimento de arco que não é uma geodésica. Se $N_{3}=0$, então

$$
T(t)=\operatorname{sen} \alpha_{0} \cos \beta(t) E_{1}+\operatorname{sen} \alpha_{0} \operatorname{sen} \beta(t) E_{2}+\cos \alpha_{0} E_{3},
$$

onde $\alpha_{0} \in(0, \pi)$.

Demonstração. Se $\gamma^{\prime}=T=T_{1} E_{1}+T_{2} E_{2}+T_{3} E_{3}$, então

$\nabla_{T} T=\left(T_{1}^{\prime}+2 m x T_{2}^{2}-2 m y T_{1} T_{2}+\ell T_{2} T_{3}\right) E_{1}+\left(T_{2}^{\prime}+2 m y T_{1}^{2}-2 m x T_{1} T_{2}-\ell T_{1} T_{3}\right) E_{2}+T_{3}^{\prime} E_{3}$.

Das fórmulas de Frenet temos que $\nabla_{T} T=\kappa_{g} N$ e, então, $N_{3}=0$ equivale a $T_{3}^{\prime}=0$, ou seja a $T_{3}=$ constante. Como $\left|T_{3}\right| \leq\|T\|=1$, se $\left|T_{3}\right|=1$ então $\nabla_{T} T=0$ que, por sua vez, implica em $\gamma$ ser uma geodésica. Segue então que $T_{3} \in(-1,1)$. Consequentemente, existe uma constante $\alpha_{0} \in(0, \pi)$ tal que $\cos \alpha_{0}=T_{3}$. Além disso, como $\|T\|=1$ então $T_{1}^{2}+T_{2}^{2}=\operatorname{sen}^{2}\left(\alpha_{0}\right)$. Podemos então definir uma função suave $\beta(t)$, tal que $\cos \beta(t) \operatorname{sen} \alpha_{0}=T_{1}$ e $\operatorname{sen} \beta(t) \operatorname{sen} \alpha_{0}=T_{2}$. Dessa forma,

$$
T(t)=\operatorname{sen} \alpha_{0} \cos \beta(t) E_{1}+\operatorname{sen} \alpha_{0} \operatorname{sen} \beta(t) E_{2}+\cos \alpha_{0} E_{3}
$$

Usando a expressão (4.12) para $T$, podemos estabelecer o resultado central desta seção.

Teorema 4.3.4. Seja $\left(M, g_{\ell, m}\right)$ o espaço de Biachi-Cartan-Vranceanu com $m \neq 0$ e $\ell^{2}-4 m \neq 0$. Assumindo que $\delta=\ell^{2}+\left(16 m-5 \ell^{2}\right) \operatorname{sen}^{2}\left(\alpha_{0}\right) \geq 0, \alpha_{0} \in(0, \pi)$, e denotando por $2 \omega_{1,2}=$ $-\ell \cos \alpha_{0} \pm \sqrt{\delta}$, então, as equações paramétricas de qualquer curva bi-harmônica própria de $\left(M, g_{\ell, m}\right)$ são de um dos três tipos listados abaixo.

Tipo I:

$$
\left\{\begin{array}{l}
x(t)=b \operatorname{sen} \alpha_{0} \operatorname{sen} \beta(t)+c, \quad b, c \in \mathbb{R}, \quad b>0, \\
y(t)=-b \operatorname{sen} \alpha_{0} \cos \beta(t)+d, \quad d \in \mathbb{R}, \\
z(t)=\frac{\ell}{4 m} \beta(t)+\frac{1}{4 m}\left[\left(4 m-\ell^{2}\right) \cos \alpha_{0}-\ell \omega_{1,2}\right] t
\end{array}\right.
$$


onde $\beta$ é uma solução não constante da seguinte EDO:

$$
\beta^{\prime}+2 m d \operatorname{sen} \alpha_{0} \cos \beta-2 m c \operatorname{sen} \alpha_{0} \operatorname{sen} \beta=\ell \cos \alpha_{0}+2 m b \operatorname{sen}^{2}\left(\alpha_{0}\right)+\omega_{1,2}
$$

e as constantes satisfazem

$$
c^{2}+d^{2}=\frac{b}{m}\left\{\left(\ell \cos \alpha_{0}+\omega_{1,2}-\frac{1}{b}\right)+m b \operatorname{sen}^{2} \alpha_{0}\right\} .
$$

Tipo II: $S e \beta=\beta_{0}=$ constante e $\cos \beta_{0} \operatorname{sen} \beta_{0} \neq 0$, as equações paramétricas são

$$
\left\{\begin{array}{l}
x(t)=x(t) \\
y(t)=x(t) \tan \beta_{0}+a \\
z(t)=\frac{1}{4 m}\left[\left(4 m-\ell^{2}\right) \cos \alpha_{0}-\ell \omega_{1,2}\right] t+b, \quad b \in \mathbb{R}
\end{array}\right.
$$

onde

$$
a=\frac{\omega_{1,2}+\ell \cos \alpha_{0}}{2 m \operatorname{sen} \alpha_{0} \cos \beta_{0}}
$$

e $x(t)$ é solução da seguinte EDO:

$$
x^{\prime}=\left(1+m\left(x^{2}+\left(x \tan \beta_{0}+a\right)^{2}\right)\right) \operatorname{sen} \alpha_{0} \cos \beta_{0} .
$$

Tipo III: $S e \cos \beta_{0} \operatorname{sen} \beta_{0}=0$, a menos de trocar $x \operatorname{com} y$, então $\cos \beta_{0}=0$ e as equações paramétricas são dadas por:

$$
\left\{\begin{array}{l}
x(t)=x_{0} \mp \frac{\omega_{1,2}+\ell \cos \alpha_{0}}{2 m \operatorname{sen} \alpha_{0}} \\
y(t)=y(t) \\
z(t)=\frac{1}{4 m}\left[\left(4 m-\ell^{2}\right) \cos \alpha_{0}-\ell \omega_{1,2}\right] t+b, \quad b \in \mathbb{R},
\end{array}\right.
$$

onde $y(t)$ é uma solução da seguinte EDO:

$$
y^{\prime}= \pm\left(1+m\left[x_{0}^{2}+y^{2}\right]\right) \operatorname{sen} \alpha_{0}
$$

Demonstração. Seja $\gamma(t)=(x(t), y(t), z(t))$ uma curva parametrizada pelo comprimento de arco. Faremos uso das fórmulas de Frenet, do Corolário 4.3.2 e do Lema 4.3.3. A derivada covariante do campo vetorial $T$, dado em (4.12), é

$$
\begin{aligned}
\nabla_{T} T & =-\beta^{\prime} \operatorname{sen} \alpha_{0} \operatorname{sen} \beta E_{1}+\beta^{\prime} \operatorname{sen} \alpha_{0} \cos \beta E_{2}+\operatorname{sen} \alpha_{0} \cos \beta \nabla_{T} E_{1} \\
& +\operatorname{sen} \alpha_{0} \operatorname{sen} \beta \nabla_{T} E_{2}+\cos \alpha_{0} \nabla_{T} E_{3} \\
& =\left[-\beta^{\prime} \operatorname{sen} \alpha_{0} \operatorname{sen} \beta-2 m y \operatorname{sen}^{2} \alpha_{0} \operatorname{sen} \beta \cos \beta+2 m x \operatorname{sen}^{2} \alpha_{0} \operatorname{sen}^{2} \beta\right. \\
& \left.+\ell \cos \alpha_{0} \operatorname{sen} \alpha_{0} \operatorname{sen} \beta\right] E_{1} \\
& +\left[\beta^{\prime} \operatorname{sen} \alpha_{0} \cos \beta+2 m y \operatorname{sen}^{2} \alpha_{0} \cos ^{2} \beta-2 m x \operatorname{sen}^{2} \alpha_{0} \operatorname{sen} \beta \cos \beta\right. \\
& \left.-\ell \cos \alpha_{0} \operatorname{sen} \alpha_{0} \cos \beta\right] E_{2} .
\end{aligned}
$$


Igualando tal expressão com $\kappa_{g} N$, obtemos

$$
\kappa_{g}^{2}=\left(\beta^{\prime}+2 m y \operatorname{sen} \alpha_{0} \cos \beta-2 m x \operatorname{sen} \alpha_{0} \operatorname{sen} \beta-\ell \cos \alpha_{0}\right)^{2} \operatorname{sen}^{2} \alpha_{0}
$$

e consequentemente (sendo $\alpha_{0} \in(0, \pi)$ ), resulta

$$
\kappa_{g}=\left|\beta^{\prime}+2 m y \operatorname{sen} \alpha_{0} \cos \beta-2 m x \operatorname{sen} \alpha_{0} \operatorname{sen} \beta-\ell \cos \alpha_{0}\right| \operatorname{sen} \alpha_{0}
$$

Neste ponto assumimos que

$$
\omega:=\beta^{\prime}+2 m y \operatorname{sen} \alpha_{0} \cos \beta-2 m x \operatorname{sen} \alpha_{0} \operatorname{sen} \beta-\ell \cos \alpha_{0}>0 .
$$

Observe que se $\omega=0$ então $\gamma$ é geodésica e se $\omega<0$ obtemos as mesmas soluções, portanto não estamos perdendo a generalidade. Dessa forma, temos

$$
\kappa_{g}=\omega \operatorname{sen} \alpha_{0}
$$

$\mathrm{e}$

$$
N=-\operatorname{sen} \beta E_{1}+\cos \beta E_{2} .
$$

Logo

$$
B=T \wedge N=-\cos \alpha_{0} \cos \beta E_{1}-\cos \alpha_{0} \operatorname{sen} \beta E_{2}+\operatorname{sen} \alpha_{0} E_{3} .
$$

Vamos agora derivar $B$ para obter a expressão da torsão geodésica $\tau_{g}$. Como

$$
\nabla_{T} B=\left(-\omega \cos \alpha-\frac{\ell}{2}\right) N
$$

e por outro lado, $\nabla_{T} B=\tau_{g} N$, segue que

$$
\tau_{g}=-\omega \cos \alpha_{0}-\frac{\ell}{2}
$$

Para obtermos as equações explícitas para $\gamma(t)=(x(t), y(t), z(t))$, devemos integrar o sistema $\frac{d \gamma}{d t}=T$. Em nosso caso

$$
\begin{aligned}
T(t) & =\operatorname{sen} \alpha_{0} \cos \beta(t) E_{1}+\operatorname{sen} \alpha_{0} \operatorname{sen} \beta(t) E_{2}+\cos \alpha_{0} E_{3} \\
& =\operatorname{sen} \alpha_{0} \cos \beta(t)\left(F \frac{\partial}{\partial x}-\frac{\ell}{2} y \frac{\partial}{\partial z}\right)+\operatorname{sen} \alpha_{0} \operatorname{sen} \beta(t)\left(F \frac{\partial}{\partial y}+\frac{\ell}{2} x \frac{\partial}{\partial z}\right)+\cos \alpha_{0}\left(\frac{\partial}{\partial z}\right),
\end{aligned}
$$

o que dá origem ao sistema

$$
\left\{\begin{array}{l}
\frac{x^{\prime}}{1+m\left(x^{2}+y^{2}\right)}=\operatorname{sen} \alpha_{0} \cos \beta \\
\frac{y^{\prime}}{1+m\left(x^{2}+y^{2}\right)}=\operatorname{sen} \alpha_{0} \operatorname{sen} \beta \\
z^{\prime}=\cos \alpha_{0}+\frac{\ell}{2} \operatorname{sen} \alpha_{0}(x \operatorname{sen} \beta-y \cos \beta) .
\end{array}\right.
$$


Assumiremos agora que $\beta^{\prime} \neq 0$, ou seja, consideraremos soluções do Tipo I. Derivando (4.20), obtemos

$$
0=\kappa_{g}^{\prime}=\omega^{\prime} \operatorname{sen} \alpha_{0}
$$

$\operatorname{logo} \omega^{\prime}=0$. Substituindo $\omega$ e utilizando as duas primeiras equações de (4.23) resulta que

$$
\beta^{\prime \prime}+\frac{2 m y^{\prime} x^{\prime}}{1+m\left(x^{2}+y^{2}\right)}-\frac{\beta^{\prime} 2 m y y^{\prime}}{1+m\left(x^{2}+y^{2}\right)}-\frac{2 m y^{\prime} x^{\prime}}{1+m\left(x^{2}+y^{2}\right)}-\frac{\beta^{\prime} 2 m x x^{\prime}}{1+m\left(x^{2}+y^{2}\right)}=0
$$

e, então,

$$
\frac{\beta^{\prime \prime}\left(1+m\left(x^{2}+y^{2}\right)\right)-\beta^{\prime}\left(2 m y y^{\prime}+2 m x x^{\prime}\right)}{1+m\left(x^{2}+y^{2}\right)}=0 .
$$

Assim

$$
\frac{d}{d t}\left(\frac{\beta^{\prime}}{1+m\left(x^{2}+y^{2}\right)}\right)=0
$$

e

$$
1+m\left(x^{2}+y^{2}\right)=b \beta^{\prime}, \quad b>0
$$

Substituindo (4.24) em (4.23), conseguimos o sistema

$$
\left\{\begin{array}{l}
x^{\prime}=b \beta^{\prime} \operatorname{sen} \alpha_{0} \cos \beta \\
y^{\prime}=b \beta^{\prime} \operatorname{sen} \alpha_{0} \operatorname{sen} \beta \\
z^{\prime}=\cos \alpha_{0}-\frac{\ell}{2} \operatorname{sen} \alpha_{0}(x \operatorname{sen} \beta-y \cos \beta),
\end{array}\right.
$$

do qual resulta que

$$
\left\{\begin{array}{l}
x(t)=b \operatorname{sen} \alpha_{0} \operatorname{sen} \beta(t)+c \\
y(t)=-b \operatorname{sen} \alpha_{0} \cos \beta(t)+d \\
z(t)=\left(\cos \alpha_{0}+\frac{\ell b}{2} \operatorname{sen}^{2} \alpha_{0}\right) t+\frac{l}{2} \int\left(c \operatorname{sen} \alpha_{0} \operatorname{sen} \beta(t)-d \operatorname{sen} \alpha_{0} \cos \beta(t)\right) d t .
\end{array}\right.
$$

Para determinar $\beta$ substituímos na última equação de (4.11) os valores de $\kappa_{g}$, $\tau_{g}$ e $B_{3}$ obtidos em (4.20), (4.22) e (4.21), respectivamente. Isto nos dá

$$
\omega^{2}+\omega l \cos \alpha_{0}+\left(\ell^{2}-4 m\right) \operatorname{sen}^{2} \alpha_{0}=0
$$

Como assumimos que $\delta=\ell^{2}+\left(16 m-5 \ell^{2}\right) \operatorname{sen}^{2} \alpha_{0} \geq 0$, então as soluções de (4.26) são

$$
\omega_{1,2}=\frac{-\ell \cos \alpha_{0} \pm \sqrt{\delta}}{2}
$$

que são sempre diferente de zero. Como assumimos que $\omega$ é uma constante positiva, temos que escolher a raiz positiva de (4.26). Se $\omega$ fosse negativo, ocorre uma troca de sinais em $\kappa_{g}, \tau_{g}$ e 
$B_{3}$, contudo chegaríamos a mesma equação (4.26), portanto aproveitamos as duas soluções de (4.26).

Substituindo os valores de $x$ e $y$ (obtidos em (4.25)) na (4.19), encontramos

$$
\beta^{\prime}-2 m \operatorname{sen} \alpha_{0}\left(b \operatorname{sen} \alpha_{0} \cos ^{2} \beta-d \cos \beta+b \operatorname{sen}_{a} l \operatorname{sen}^{2} \beta+c \operatorname{sen} \beta\right)=\ell \cos \alpha_{0}+\omega_{1,2},
$$

de onde segue

$$
\beta^{\prime}-2 m\left(c \operatorname{sen} \alpha_{0} \operatorname{sen} \beta-d \operatorname{sen} \alpha_{0} \cos \beta\right)=\ell \cos \alpha_{0}+2 m b \operatorname{sen}^{2} \alpha_{0}+\omega_{1,2} .
$$

Então, tomando a integral da ultima equação, o valor de $z$ dado em (4.25) se torna o desejado. Para o caso das soluções Tipo I, substituindo (4.25) e (4.24) em (4.27),

$$
\begin{aligned}
b\left(\ell \cos \alpha_{0}+2 \nabla b \operatorname{sen}^{2} \alpha_{0}+\omega_{1,2}\right) & =1+m\left(x^{2}+y^{2}\right)+2 b m d \operatorname{sen} \alpha_{0} \cos \beta \\
& -2 b m c \operatorname{sen} \alpha_{0} \operatorname{sen} \beta \\
& =1+m\left[\left(b \operatorname{sen} \alpha_{0} \operatorname{sen} \beta(t)+c\right)^{2}\right. \\
& \left.+\left(-b \operatorname{sen} \alpha_{0} \cos \beta(t)+d\right)^{2}\right] \\
& +2 b m d \operatorname{sen} \alpha_{0} \cos \beta-2 b m c \operatorname{sen} \alpha_{0} \operatorname{sen} \beta
\end{aligned}
$$

e, então, temos

$$
c^{2}+d^{2}=\frac{b}{m}\left\{\left(\ell \cos \alpha_{0}-\frac{1}{b}\right)+m b \operatorname{sen}^{2} \alpha_{0}\right\} .
$$

Para o Tipo II, usamos $\beta=\beta_{0}=$ constante nas duas primeiras equações de (4.23) obtendo:

$$
\begin{aligned}
& x^{\prime}=\left[1+m\left(x^{2}+y^{2}\right)\right] \operatorname{sen} \alpha_{0} \cos \beta_{0}, \\
& y^{\prime}=\left[1+m\left(x^{2}+y^{2}\right)\right] \operatorname{sen} \alpha_{0} \operatorname{sen} \beta_{0} .
\end{aligned}
$$

Como sen $\beta_{0} \cos \beta \neq 0$,

$$
y^{\prime}=x^{\prime} \tan \beta_{0}
$$

portanto

$$
y=x \tan \beta_{0}+a
$$

Substituindo (4.29) em (4.28) temos

$$
x^{\prime}=\left[1+m\left(x^{2}+\left(x \tan \beta_{0}+a\right)^{2}\right)\right] \operatorname{sen} \alpha_{0} \cos \beta_{0} .
$$

Além disso, usando (4.29) em (4.19),

$$
\begin{aligned}
\omega_{1,2}+\ell \cos \alpha_{0} & =2 m \operatorname{sen} \alpha_{0}\left(y \cos \beta_{0}-x \operatorname{sen} \beta_{0}\right) \\
& =2 m \operatorname{sen} \alpha_{0}\left[\left(x \tan \beta_{0}+a\right) \cos \beta_{0}-x \operatorname{sen} \beta_{0}\right],
\end{aligned}
$$


$\log 0$

$$
a=\frac{\omega_{1,2}+\ell \cos \alpha_{0}}{2 m \operatorname{sen} \alpha_{0} \cos \beta_{0}}
$$

Para determinar a expressão de $z$, utilizamos (4.29) e (4.30) na última equação do sistema (4.23) e integramos, obtendo

$$
z=\frac{1}{4 m}\left[\left(4 m-l^{2}\right) \cos \alpha_{0}-l \omega_{1,2}\right] t+b, \quad b \in \mathbb{R} .
$$

Para as soluções Tipo III, ao supormos sen $\beta_{0} \cos \beta_{0}=0$ teremos que analisar dois casos.

Se $\cos \beta_{0}=0$, então da primeira equação de (4.23) resulta que $x(t)=x_{0} \in \mathbb{R}$. Além disso, como sen $\beta_{0}= \pm 1$, da segunda equação de (4.23) obtemos

$$
y^{\prime}= \pm\left[1+m\left(x_{0}^{2}+y^{2}\right)\right] \operatorname{sen} \alpha_{0}
$$

Usando (4.19) obtemos que

$$
x_{0}=\mp\left(\frac{\omega_{1,2}+\ell \cos \alpha_{0}}{2 m \operatorname{sen} \alpha_{0}}\right) .
$$

Por fim, determinamos a expressão de $z(t)$ substituindo (4.31) na última equação de (4.23) e integrando, obtendo

$$
z(t)=\frac{1}{4 m}\left[\left(4 m-\ell^{2}\right) \cos \alpha_{0}-\ell \omega_{1,2}\right] t+b, \quad b \in \mathbb{R} .
$$

Se $\operatorname{sen} \beta=0$, há uma troca nos papéis de $x$ e $y$ e o resultado segue de forma análoga.

Observação 4.3.5. A condição $m \neq 0$ foi utilizada pela primeira vez na demonstração do caso das soluções Tipo I do Teorema 4.3.4, na obtenção de $z(t)$ a partir da EDO que define $\beta$, de forma que todos os resultados obtidos anteriormente continuam válidos se $m=0$.

\subsection{Equações explícitas das curvas bi-harmônicas próprias em $\mathbb{H}_{3}$}

Passaremos agora a estudar as curvas bi-harmônicas próprias do espaço de Heisenberg $\mathbb{H}_{3}$, ou seja da variedade $\left(M, g_{\ell, m}\right) \operatorname{com} \ell=1$ e $m=0$. Tais resultados foram obtidos por R. Caddeo, C. Oniciuc e P. Piu em [12].

O grupo de Heisenberg $\mathbb{H}_{3}$ pode ser visto como o espaço Euclidiano $\mathbb{R}^{3}$ com a multiplicação

$$
L_{(\tilde{x}, \tilde{y}, \tilde{z})}(x, y, z)=(\tilde{x}, \tilde{y}, \tilde{z})(x, y, z)=\left(\tilde{x}+x, \tilde{y}+y, \tilde{z}+z+\frac{1}{2} \tilde{x} y-\frac{1}{2} \tilde{y} x\right)
$$


e munido da métrica Riemanniana $g$ dada por

$$
g=d x^{2}+d y^{2}+\left(d z+\frac{y}{2} d x-\frac{x}{2} d y\right)^{2} .
$$

A métrica $g$ é invariante à esquerda e é isométrica as demais, também muito conhecidas, que são invariantes com respeito a uma composição com a multiplicação de matrizes de Heisenberg.

Em seguida é dado o resultado que classifica as curvas bi-harmônicas de $\mathbb{H}_{3}$.

Teorema 4.4.1. As equações paramétricas de uma curva bi-harmônica própria qualquer de $\left(\mathbb{H}_{3}, g\right)$, parametrizada pelo comprimento de arco, são dadas por:

$$
\left\{\begin{array}{l}
x(t)=\frac{1}{A} \operatorname{sen} \alpha_{0} \operatorname{sen}(A t+a)+b \\
y(t)=-\frac{1}{A} \operatorname{sen} \alpha_{0} \cos (A t+a)+c \\
z(t)=\left(\cos \alpha_{0}+\frac{\operatorname{sen}^{2} \alpha_{0}}{2 A}\right) t-\frac{b}{2 A} \operatorname{sen} \alpha_{0} \cos (A t+a)-\frac{c}{2 A} \operatorname{sen} \alpha_{0} \operatorname{sen}(A t+a)+d,
\end{array}\right.
$$

onde

$$
A=\frac{\cos \alpha_{0} \pm \sqrt{5 \cos ^{2} \alpha_{0}-4}}{2}, \quad \alpha_{0} \in\left(0, \arccos \frac{2 \sqrt{5}}{5}\right] \cup\left[\arccos \left(-\frac{2 \sqrt{5}}{5}\right), \pi\right)
$$

e $a, b, c, d \in \mathbb{R}$.

Demonstração. Seja $\gamma: I \rightarrow \mathbb{H}_{3}, \gamma(t)=(x(t), y(t), z(t))$, uma curva bi-harmônica própria parametrizada pelo comprimento de arco. Da equação (4.27) temos que

$$
\beta^{\prime}=\frac{\cos \alpha_{0} \pm \sqrt{5 \cos ^{2} \alpha_{0}-4}}{2}=A .
$$

Impondo a condição $5 \cos ^{2} \alpha_{0} \geq 4$ (para que tenhamos soluções reais), $\beta(t)=A t+a$, com $a \in \mathbb{R}$.

Para obtermos as equações explícitas de $\gamma$ basta integrar o sistema $\gamma^{\prime}=T$, que neste caso é dado por:

$$
\left\{\begin{array}{l}
x^{\prime}(t)=\operatorname{sen} \alpha_{0} \cos (A t+a) \\
y^{\prime}(t)=\operatorname{sen} \alpha_{0} \operatorname{sen}(A t+a) \\
z^{\prime}(t)=\cos \alpha_{0}+\frac{1}{2} \operatorname{sen} \alpha_{0}[\operatorname{sen}(A t+a) x(t)-\cos (A t+a) y(t)]
\end{array}\right.
$$

já que substituindo as expressões de $E_{1}, E_{2}$ e $E_{3}$ em (4.12) obtemos

$$
\begin{aligned}
T= & \operatorname{sen} \alpha_{0} \cos (\beta) \frac{\partial}{\partial x}+\operatorname{sen} \alpha_{0} \operatorname{sen}(\beta) \frac{\partial}{\partial y} \\
& +\left[\cos \alpha_{0}+\frac{1}{2} \operatorname{sen} \alpha_{0}(\operatorname{sen}(A t+a) x(t)-\cos (A t+a) y(t))\right] \frac{\partial}{\partial z} .
\end{aligned}
$$


Observação 4.4.2. As curvas bi-harmônicas de $\mathbb{H}_{3}$ podem ser obtidas pela intersecção das superfícies $S$ e $S^{\prime}$, parametrizadas (respectivamente) por:

$$
\begin{gathered}
\left\{\begin{array}{l}
x(u, v)=\frac{1}{A} \operatorname{sen} \alpha_{0} \cos (A u+a)+b \\
y(u, v)=-\frac{1}{A} \operatorname{sen} \alpha_{0} \operatorname{sen}(A u+a)+c \\
z(u, v)=v
\end{array}\right. \\
\left\{\begin{array}{l}
x(u, v)=\frac{v}{A} \operatorname{sen} \alpha_{0} \cos (A u+a)+b \\
y(u, v)=-\frac{v}{A} \operatorname{sen} \alpha_{0} \operatorname{sen}(A u+a)+c \\
z(u, v)=\left(\cos \alpha_{0}+\frac{\operatorname{sen}^{2} \alpha_{0}}{2 A}\right) u+\frac{b}{2} y(u, v)-\frac{c}{2} x(u, v)+d .
\end{array}\right.
\end{gathered}
$$

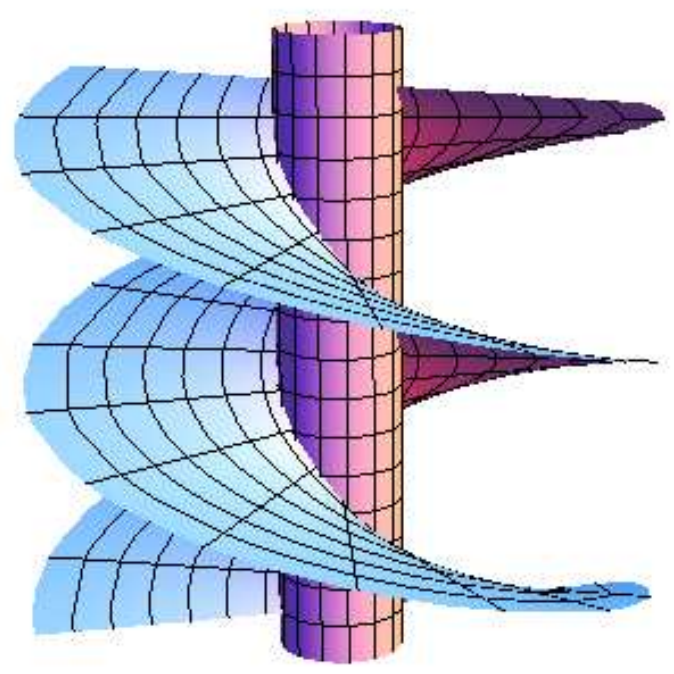

Figura 4.2: Intersecção entre as superfícies $S$ e $S^{\prime}$.

A superfície $S$ possui curvatura média constante diferente de zero e é o cilindro circular reto com eixo paralelo ao eixo de rotação de $\mathbb{H}_{3}$ no ponto $(b, c, 0)$ e com a diretriz o círculo no plano $z=0$ centrado neste ponto; esse círculo tem curvatura geodésica constante também em $\mathbb{H}_{3}$. A superfície $S^{\prime}$ é um helicóide que é minimal no grupo de Heisenberg, como pode ser facilmente constatado pelo uso das fórmula dadas por M. Bekkar em [2]. Além disso, as curvas bi-harmônicas são geodésicas do cilindro. 
Observação 4.4.3. Denotamos por $\gamma$ a curva dada em (4.34) com $b=c=d=0$, e por $\widetilde{\gamma}$ a curva (4.34) com $b^{2}+c^{2}>0$. É claro que as duas são hélices de $\mathbb{H}_{3}$. Toda curva bi-harmônica $\widetilde{\gamma}$ pode ser obtida de $\gamma$ por meio de uma translação à esquerda, isto é $\widetilde{\gamma}=L_{(b, c, d)} \circ \gamma$. Observa-se também que $\gamma$ é uma hélice em $\mathbb{R}^{3}$, mas $\widetilde{\gamma}$ não é.

\subsection{Curvas bi-harmônicas próprias da esfera $\mathbb{S}^{3}$}

Nesta seção serão classificadas as curvas bi-harmônicas não geodésicas da esfera unitária tridimensional. Iniciaremos fazendo algumas considerações inerentes as variedades tridimensionais de curvatura seccional constante.

Considere, então, $\left(M^{3}, g\right)$ uma variedade Riemanniana tridimensional de curvatura seccional constante e igual $K$. Seja $\gamma: I \rightarrow\left(M^{3}, g\right)$ uma curva diferenciável parametrizada pelo comprimento de arco e $\{T, N, B\}$ o referencial de Frenet ao longo de $\gamma$ como em (3.1), com $F_{1}=T$, $F_{2}=B$ e $F_{3}=N$. Valem, então, as relações:

$$
\left\{\begin{array}{l}
\nabla_{T} T=\kappa_{g} N \\
\nabla_{T} N=-\kappa_{g} T+\tau_{g} B \\
\nabla_{T} B=-\tau_{g} N
\end{array}\right.
$$

onde $\kappa_{g}=\|\tau(\gamma)\|=\left\|\nabla_{T} T\right\|$ é a curvatura geodésica de $\gamma$ e $\tau_{g}$ é sua torsão geodésica. Do sistema (3.2), concluímos que uma curva de $\left(M^{3}, g\right)$ é bi-harmônica se, e somente se,

$$
\left\{\begin{array}{l}
\kappa_{g} \kappa_{g}^{\prime}=0 \\
\kappa_{g}^{\prime \prime}-\kappa_{g}^{3}-\kappa_{g} \tau_{g}^{2}+\kappa_{g} K=0 \\
2 \kappa_{g}^{\prime} \tau_{g}+\kappa_{g} \tau_{g}^{\prime}=0 .
\end{array}\right.
$$

Impodo a condição de que a curva não seja uma geodésica, obtemos

$$
\left\{\begin{array}{l}
\kappa_{g}=\text { constante } \neq 0 \\
\kappa_{g}^{2}+\tau_{g}^{2}=K \\
\tau_{g}=\text { constante }
\end{array}\right.
$$

Consequentemente, podemos enunciar o resultado seguinte.

Proposição 4.5.1. Seja $\gamma: I \rightarrow\left(M^{3}, g\right)$ uma curva bi-harmônica própria em uma variedade Riemanniana com curvatura seccional constante $K$. Se $K$ é não positiva, então toda curva bi-harmônica é uma geodésica de $\left(M^{3}, g\right)$. 
Desta proposição segue que o estudo de curvas bi-harmônicas em variedades tridimensionais com curvatura seccional constante passa a ter interesse apenas para o caso em que $K>0$. Por essa razão passaremos a estudar o caso da esfera $\mathbb{S}^{3}$. Faremos primeiro algumas considerações.

Seja $(M, h)$ uma hipersuperfície de $\mathbb{R}^{n+1}$, munida da métrica induzida. Denotaremos por $\bar{\nabla}$ e $\nabla$ as conexão riemannianas de $\mathbb{R}^{n+1}$ e $M$, respectivamente. Seja $p$ um ponto de $M, \eta \in\left(T_{p} M\right)^{\perp}$ um vetor unitário e $N$ uma extensão local de $\eta$, unitária e normal a $M$. Sendo $g: M \rightarrow \mathbb{S}^{n}$, a aplicação normal de Gauss, $T_{p} M$ e $T_{g(p)} \mathbb{S}^{n}$ são paralelos e, portanto, serão identificados. Denotaremos por II a segunda forma fundamental de $M$ e $S_{\eta}$ o operador de forma. Com isso, vale

$$
d g_{p}\left(X_{p}\right)=\frac{d}{d t}(N \circ c(t))_{t=0}=\bar{\nabla}_{X_{p}} N=\left(\bar{\nabla}_{X_{p}} N\right)^{T}=-S_{\eta}\left(X_{p}\right),
$$

onde $c:(-\epsilon, \epsilon) \rightarrow M$ é uma curva com $c(0)=p$ e $c^{\prime}(0)=X_{p} \in T_{p} M$. Aqui usamos o fato de que, sendo $\langle N, N\rangle=1, \bar{\nabla}_{X_{p}} N=\left(\bar{\nabla}_{X_{p}} N\right)^{T}$. Segue-se que $-S_{\eta}$ é a derivada da aplicação normal de Gauss.

Considere, agora, $M=\mathbb{S}^{n}$ (esfera unitária), $p \in \mathbb{S}^{n}$ e $\eta=-p$. Para esse caso, a aplicação de Gauss é $-I$, sendo $I$ a identidade de $\mathbb{S}^{n}$. Segue que $S_{\eta}\left(X_{p}\right)=-d g_{p}\left(X_{p}\right)=X_{p}, X_{p} \in T_{p} \mathbb{S}^{n}$ e, então,

$$
\begin{aligned}
\operatorname{II}\left(X_{p}, Y_{p}\right) & =\left\langle\operatorname{II}\left(X_{p}, Y_{p}\right), \eta\right\rangle \eta \\
& =\left\langle S_{\eta}\left(X_{p}\right), Y_{p}\right\rangle \eta \\
& =-\left\langle X_{p}, Y_{p}\right\rangle p, \quad X_{p}, Y_{p} \in T_{p} \mathbb{S}^{n} .
\end{aligned}
$$

Portanto, a equação de Gauss

$$
\nabla_{X}^{\mathbb{S}^{n}} Y=\bar{\nabla}_{X} Y+\langle X, Y\rangle p
$$

Agora estamos aptos a dar a demonstração da proposição que segue.

Proposição 4.5.2. Seja $\gamma: I \rightarrow \mathbb{S}^{3} \subset \mathbb{R}^{4}$ uma curva bi-harmônica própria parametrizada pelo comprimento de arco. Então

$$
\gamma^{i v}+2 \gamma^{\prime \prime}+\left(1-\kappa_{g}^{2}\right) \gamma=0
$$

Demonstração. Tomando a derivada covariante da segunda equação de (4.39) com respeito a $T$, temos

$$
\begin{aligned}
\nabla_{T}^{2} N & =-\kappa_{g} \nabla_{T} T+\tau_{g} \nabla_{T} B \\
& =-\left(\kappa_{g}^{2}+\tau_{g}^{2}\right) N \\
& =-N
\end{aligned}
$$


ou seja

$$
\nabla_{T}^{2} N+N=0
$$

Por outro lado, fazendo uso da equação de Gauss (4.41) em $\nabla_{T} N$ resulta que

$$
\begin{aligned}
\nabla_{T}^{2} N & =\left(\nabla_{T} N\right)^{\prime}+\left\langle T, \nabla_{T} N\right\rangle \gamma \\
& =\left(N^{\prime}+\langle T, N\rangle \gamma\right)^{\prime}-\kappa_{g} \gamma \\
& =N^{\prime \prime}-\kappa_{g} \gamma
\end{aligned}
$$

Além disso, aplicando (4.41) em $\nabla_{T} T=\kappa_{g} N$ conseguimos

$$
N=\frac{1}{\kappa_{g}}\left(\gamma^{\prime \prime}+\gamma\right)
$$

Reescrevendo (4.43) como

$$
N^{\prime \prime}-\kappa_{g} \gamma+N=0
$$

temos

$$
\left(\frac{1}{\kappa_{g}}\left(\gamma^{\prime \prime}+\gamma\right)\right)^{\prime \prime}-\kappa_{g} \gamma+\frac{1}{\kappa_{g}}\left(\gamma^{\prime \prime}+\gamma\right)=0,
$$

de onde segue o resultado.

A classificação das curvas bi-harmônicas de $\mathbb{S}^{3}$ é dada no teorema que segue:

Teorema 4.5.3. Seja $\gamma: I \rightarrow \mathbb{S}^{3} \subset \mathbb{R}^{4}$ uma curva bi-harmônica própria parametrizada pelo comprimento de arco. Então $\kappa_{g} \leq 1$ e temos dois casos:

1. $\kappa_{g}=1$ e $\gamma$ é um círculo de raio $\frac{1}{\sqrt{2}}$;

2. $0<\kappa_{g}<1$ e $\gamma$ é uma geodésica do toro de Clifford ${ }^{1}$.

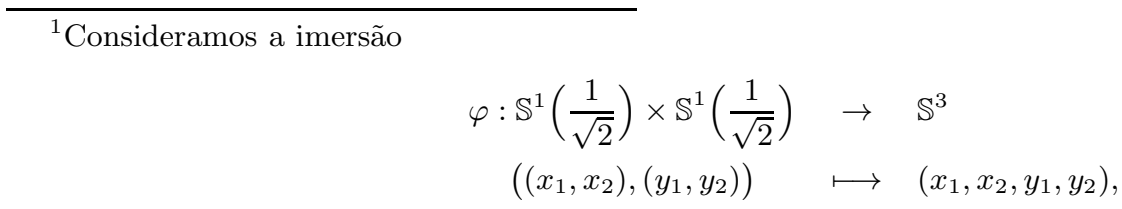

onde $x_{1}^{2}+x_{2}^{2}=y_{1}^{2}+y_{2}^{2}=\frac{1}{2}$. A subvariedade de $\mathbb{S}^{3}, \mathcal{T}=\mathbb{S}^{1}\left(\frac{1}{\sqrt{2}}\right) \times \mathbb{S}^{1}\left(\frac{1}{\sqrt{2}}\right)$, é chamada de toro de Clifford. Observe que dado um ponto $p=\left(x_{1}, x_{2}, y_{1}, y_{2}\right) \in \mathcal{T}$, os vetores

$$
V_{p}=\left(-x_{2}, x_{1}, 0,0\right) \quad \text { e } \quad W_{p}=\left(0,0,-y_{2}, y_{1}\right)
$$

constituem um base do espaço tangente em $p$ ao toro de Clifford. 
Demonstração. Da segunda equação de (4.40), segue que $\kappa_{g} \leq 1$. Temos, então, os dois casos considerados abaixo.

1. Se $\kappa_{g}=1$, então a solução geral de (4.42) é

$$
\gamma(t)=c_{1} \cos (\sqrt{2} t)+c_{2} \operatorname{sen}(\sqrt{2} t)+c_{3} t+c_{4}
$$

Como $|\gamma|^{2}=\left|\gamma^{\prime}\right|^{2}=1$, usando as relações de Frenet ${ }^{2}$ temos que $c_{3}=0$, enquanto $c_{1}, c_{2}$ e $c_{4}$ são vetores constantes e ortogonais com $\left|c_{1}\right|^{2}=\left|c_{2}\right|^{2}=\left|c_{4}\right|^{2}=\frac{1}{2}$.

Portanto, a menos de isometrias de $\mathbb{S}^{3}$, a curva $\gamma$ é da forma

$$
\gamma(t)=\left(\frac{\cos (\sqrt{2} t)}{\sqrt{2}}, \frac{\operatorname{sen}(\sqrt{2} t)}{\sqrt{2}}, d_{1}, d_{2}\right)
$$

$\operatorname{com} d_{1}^{2}+d_{1}^{2}=\left|c_{4}\right|^{2}=\frac{1}{2}$, ou seja é uma circunferência de raio $\frac{1}{\sqrt{2}}$.

2. Agora supondo $0<\kappa_{g}<1$, a solução geral de (4.42) é

$$
\gamma(t)=c_{1} \cos (a t)+c_{2} \operatorname{sen}(a t)+c_{3} \cos (b t)+c_{4} \operatorname{sen}(b t)
$$

onde $a=\sqrt{1+\kappa_{g}}$ e $b=\sqrt{1-\kappa_{g}}$. Novamente usamos $|\gamma|^{2}=1,\left|\gamma^{\prime}\right|^{2}=1$ e as relações de Frenet para concluir (com contas análogas ao caso anterior) que $\left|c_{1}\right|^{2}=\left|c_{2}\right|^{2}=\left|c_{3}\right|^{2}=$ $\left|c_{4}\right|^{2}=\frac{1}{2}$ e que tais vetores são ortogonais entre si.

Segue, então, que a solução para $0<\kappa_{g}<1$ é

$$
\gamma(t)=\left(\frac{\cos (a t)}{\sqrt{2}}, \frac{\operatorname{sen}(a t)}{\sqrt{2}}, \frac{\cos (b t)}{\sqrt{2}}, \frac{\operatorname{sen}(b t)}{\sqrt{2}}\right) .
$$

É evidente que, para este caso, $\gamma(t) \in \mathbb{S}^{1}\left(\frac{1}{\sqrt{2}}\right) \times \mathbb{S}^{1}\left(\frac{1}{\sqrt{2}}\right)$.

${ }^{2}$ De fato, de $N=\frac{1}{\kappa_{g}}\left(\gamma^{\prime \prime}+\gamma\right)$, resulta que

$$
N^{\prime}=\sqrt{2} \operatorname{sen}(\sqrt{2} t) c_{1}-\sqrt{2} \cos (\sqrt{2} t) c_{2}+c_{3} .
$$

Por outro lado, devido à segunda equação de (4.40), como $\kappa_{g}=1, \tau_{g}=0$. Então, usando (4.41), temos

$$
N^{\prime}=\nabla_{T} N=-\kappa_{g} T+\tau_{g} B=-\gamma^{\prime}=\sqrt{2} \operatorname{sen}(\sqrt{2} t) c_{1}-\sqrt{2} \cos (\sqrt{2} t) c_{2},
$$

portanto $c_{3}=0$. De $|\gamma|^{2}=1$ e $\left\langle N, \gamma^{\prime}\right\rangle=0,\left\langle\gamma^{\prime \prime}+2 \gamma, \gamma^{\prime}\right\rangle=0$; portanto

$$
\left\langle c_{4}, c_{1}\right\rangle=\left\langle c_{4}, c_{2}\right\rangle=0 .
$$

Além disso, $\left\langle\gamma, \gamma^{\prime}\right\rangle=0$ fornece $\left\langle\gamma^{\prime \prime}, \gamma\right\rangle=-1$, do qual resulta $1=\left\langle\gamma^{\prime \prime}+2 \gamma, \gamma\right\rangle=\left\langle 2 c_{4}, \gamma\right\rangle$, ou seja, $\left\langle c_{4}, c_{4}\right\rangle=\frac{1}{2}$. Por fim, usando $\left\langle c_{4}, c_{4}\right\rangle=\frac{1}{2}$ e $\langle\gamma, \gamma\rangle=1$, concluímos que $c_{1}$ é ortogonal a $c_{2}$ e $\left|c_{1}\right|^{2}=\left|c_{2}\right|^{2}=\frac{1}{2}$. 
Por último, para verificar que $\gamma$ é uma geodésica do toro de Clifford, basta calcular $\gamma^{\prime \prime}$ e constatar que suas projeções sobre a base do toro $V, W$, dada em (4.44), são nulas. 


\section{Capítulo 5}

\section{Superfícies bi-harmônicas em forma espaciais}

Neste capítulo é dada a classificação completa das superfícies bi-harmônicas próprias da variedade $\mathbb{S}^{3}$, seguindo o que foi feito em [8] por R. Caddeo, S. Montaldo e C. Oniciuc. Em princípio serão expostos resultados validos para hipersuperfícies da esfera $n$-dimensional $\mathbb{S}^{n}$, posteriormente nos restringiremos ao caso em que $n=3$.

\subsection{Resultados preliminares na esfera $\mathbb{S}^{n}$}

Seja $N(c)$ uma variedade com curvatura seccional constante $c, M^{m}$ uma subvariedade de $N(c)$ e $\mathbf{i}: M \rightarrow N(c)$ a inclusão canônica. Denotaremos por II a segunda forma fundamental da subvariedade $M \subset N(c)$, por S o operador de forma, por H o vetor curvatura média de $M$, por $\nabla^{\perp}$ a conexão normal e por $\Delta^{\perp}$ o Laplaciano generalizado, como definido em (1.45), no fibrado normal de $M$. Então, valem os resultados que seguem.

Teorema 5.1.1 ([7], [15]). A aplicação i é bi-harmônica se e somente se

$$
\left\{\begin{array}{c}
-\Delta^{\perp} \mathrm{H}-\operatorname{tr} \mathrm{II}\left(-, \mathrm{S}_{\mathrm{H}}-\right)+c m \mathrm{H}=0 \\
2 \operatorname{tr} \mathrm{S}_{\nabla_{(-)}^{\perp} \mathrm{H}}(-)+\frac{m}{2} \operatorname{grad}\left(\|\mathrm{H}\|^{2}\right)=0 .
\end{array}\right.
$$

Demonstração. Ver [7] e [15].

Ressaltamos que na demonstração do Teorema 5.1.1 o campo de bi-tensão de $\mathbf{i}$ é reescrito 
na forma

$$
\tau_{2}(i)=-m(\Delta \mathrm{H}-m c \mathrm{H})
$$

O sistema (5.1) foi estudado por B.Y. Chen e S. Ishikawa (para $c=0$ ) e por R. Caddeo, S. Montaldo e C. Oniciuc (para $c<0$ ), para provar que no caso de superfícies bi-harmônicas de $N^{3}(c), c \leq 0$, a curvatura média é constante.

Sendo que, toda subvariedade bi-harmônica de curvatura média constante numa variedade com curvatura seccional constante não positiva é harmônica (ver [31]), temos o seguinte resultado.

Teorema 5.1.2 ([7], [15]). Seja $M^{2}$ uma superfície de $N^{3}(c), c \leq 0$. Então $M$ é bi-harmônica se e somente se é minimal.

Devido a este resultado e a equação (5.2), segue a não existência de superfícies bi-harmônicas próprias em formas espaciais com $c \leq 0$. Portanto, passaremos a considerar o caso da esfera unitária. Neste caso, do Teorema 5.1.1 segue o seguinte

Corolário 5.1.3. Seja $M^{m}$ uma subvariedade de $\mathbb{S}^{n} \operatorname{com} \nabla^{\perp} \mathrm{H}=0$. Então a inclusão i é bi-harmônica se e somente se

$$
m \mathrm{H}=\operatorname{tr} \mathrm{II}\left(-, \mathrm{S}_{\mathrm{H}}-\right) .
$$

Demonstração. A demonstração segue do sistema (5.1), observando que

$$
\operatorname{grad}\left(\|\mathrm{H}\|^{2}\right)=\sum\left(e_{i}\langle\mathrm{H}, \mathrm{H}\rangle\right) e_{i}=2\left\langle\nabla_{e_{i}}^{\perp} \mathrm{H}, \mathrm{H}\right\rangle e_{i}
$$

sendo $\left\{e_{i}\right\}$ um referencial local geodésico.

Proposição 5.1.4. Seja $M$ uma hipersuperfície de $\mathbb{S}^{n}$. Então a inclusão i é bi-harmônica se e somente se

$$
\left\{\begin{array}{l}
\Delta^{\perp} \mathrm{H}=\left(m-\|\mathrm{II}\|^{2}\right) \mathrm{H} \\
2 \operatorname{tr} \mathrm{S}_{\nabla_{(-)}^{\perp} \mathrm{H}}(-)+\frac{m}{2} \operatorname{grad}\left(\|\mathrm{H}\|^{2}\right)=0 .
\end{array}\right.
$$

Demonstração. Basta observar que

$$
\operatorname{tr} \mathrm{II}\left(-, \mathrm{S}_{\mathrm{H}}-\right)=\frac{1}{m}(\operatorname{tr} \mathrm{S})\|\mathrm{II}\|^{2} \eta=\|\mathrm{II}\|^{2} \mathrm{H},
$$

e o resultado segue do Teorema 5.1.1. 
Também, temos a seguinte

Proposição 5.1.5. Seja

$$
M=\mathbb{S}^{m}(a) \times\{b\}=\left\{p=\left(x_{1}, \ldots, x_{m+1}, b\right) ;\left(x_{1}\right)^{2}+\ldots+\left(x_{m+1}\right)^{2}=a^{2}, a^{2}+b^{2}=1,0<a<1\right\}
$$

uma hiperesfera paralela de $\mathbb{S}^{m+1}$. Então $M$ é uma subvariedade bi-harmônica de $\mathbb{S}^{m+1}$ se e somente se $a=\frac{1}{\sqrt{2}}$ e $b= \pm \frac{1}{\sqrt{2}}$.

Demonstração. Observe que

$$
\Gamma(T M)=\left\{X=\left(X_{1}, \ldots, X_{m+1}, 0\right) \in \mathbb{R}^{m+2} \mid x_{1} X_{1}+\ldots+x_{m+1} X_{m+1}=0\right\}
$$

Além disso, indicando por $\xi=\left(x_{1}, \ldots, x_{m+1},-\frac{a^{2}}{b}\right)$, segue que

$$
\langle\xi, X\rangle=\langle\xi, p\rangle=0, \quad\|\xi\|^{2}=a^{2}+\frac{a^{4}}{b^{2}}=c^{2}, \quad c>0,
$$

e, então, $\xi$ é uma seção do fibrado normal de $M$ em $\mathbb{S}^{m+1}$. Pondo $\eta=\frac{1}{c} \xi$, da equação (4.41), resulta que

$$
\begin{aligned}
\nabla_{X}^{\mathbb{S}^{m+1}} \eta & =\nabla_{X}^{\perp} \eta-\mathrm{S}_{\eta}(X) \\
& =\frac{1}{c} \nabla_{X}^{\mathbb{S}^{m+1}} \xi=\frac{1}{c}\left(\nabla_{X}^{\mathbb{R}^{m+2}} \xi+\langle\xi, X\rangle p\right) \\
& =\frac{1}{c} \nabla_{\left(X_{1}, \ldots, X_{m+1}, 0\right)}^{\mathbb{R}^{m+2}}\left(x_{1}, \ldots, x_{m+1},-\frac{a^{2}}{b}\right) \\
& =\frac{1}{c} X .
\end{aligned}
$$

Logo $\nabla \frac{1}{X} \eta=0$ e $\mathrm{S}_{\eta}=-\frac{1}{c} \mathrm{I}$. Portanto, o vetor curvatura média é

$$
\mathrm{H}=\frac{1}{m}\left(\operatorname{tr} \mathrm{S}_{\eta}\right) \eta=-\frac{1}{c} \eta
$$

e consequentemente

$$
\mathrm{S}_{\mathrm{H}}=\mathrm{S}_{-\frac{1}{c} \eta}=\frac{1}{c^{2}} \mathrm{I} .
$$

Do Corolário 5.1.3 concluímos que $M$ é bi-harmônica se e somente se $c^{2}=1$, ou seja, se e somente se $a=\frac{1}{\sqrt{2}}$ e $b= \pm \frac{1}{\sqrt{2}}$.

\subsection{Superfícies bi-harmônicas da esfera $\mathbb{S}^{3}$}

A partir de agora, restringiremos nossa atenção as superfícies da esfera tridimensional $\mathbb{S}^{3}$. Da Proposição 5.1 .5 vemos que $M=\mathbb{S}^{2}\left(\frac{1}{\sqrt{2}}\right) \times\left\{ \pm \frac{1}{\sqrt{2}}\right\}$ é uma superfície bi-harmônica não harmônica de $\mathbb{S}^{3}$. 
Na Seção 4.5 vimos que curvas bi-harmônicas próprias de $\mathbb{S}^{3}$ devem ter curvatura geodésica constante não nula. O teorema que segue mostra que o mesmo vale para a norma do vetor curvatura média de superfícies bi-harmônicas não harmônicas de $\mathbb{S}^{3}$.

Teorema 5.2.1 ([8]). Seja $M$ uma superfície de $\mathbb{S}^{3}$. Então $M$ é uma subvariedade bi-harmônica não harmônica se e somente se $\|\mathrm{H}\|$ é constante não nula e $\|\mathrm{II}\|^{2}=2$.

Demonstração. Suponha que $M$ seja uma subvariedade de $\mathbb{S}^{3}$ bi-harmônica. Seja $\left\{e_{1}, e_{2}\right\}$ uma base local de campos de vetores ortonormais em $M$ e seja $\eta$ um campo vetores unitário normal. Escrevemos o vetor curvatura média na forma $\mathrm{H}=f \eta$, onde $f \in C^{\infty}(M)$ e $f>0$. Neste caso o sistema (5.4) se torna

$$
\left\{\begin{array}{l}
-\triangle^{M} f=\left(2-\left\|\mathrm{S}_{\eta}\right\|^{2}\right) f, \\
\mathrm{~S}_{\eta}(\operatorname{grad} f)+f \operatorname{grad} f=0 .
\end{array}\right.
$$

De fato, como $M$ é uma hipersuperfície temos $\left\|\mathrm{S}_{\eta}\right\|^{2}=\|\mathrm{II}\|^{2}$ e, além disso,

$$
\begin{aligned}
\nabla^{\perp} \mathrm{H} & =-\sum_{i}\left(\nabla_{e_{i}}^{\perp} \nabla_{e_{i}}^{\perp}\right) f \eta \\
& =-\sum_{i} e_{i}\left(e_{i}(f)\right) \eta+2 e_{i}(f) \nabla_{e_{i}}^{\perp} \eta+f \nabla_{e_{i}}^{\perp} \nabla_{e_{i}}^{\perp} \eta .
\end{aligned}
$$

Como $\nabla_{e_{i}}^{\perp} \eta=0$, pela equação (1.7) obtemos a primeira equação do sistema (5.6). Quanto à segunda equação de (5.6), temos que

$$
\sum_{i} \mathrm{~S}_{\nabla_{e_{i}}^{\perp} \mathrm{H}} e_{i}=\mathrm{S}_{\eta}\left(e_{i}(f) e_{i}\right)+\mathrm{S}_{\nabla_{e_{i}}^{\perp} \eta} e_{i}
$$

e, então, o resultado segue de (1.3) e de $\nabla_{e_{i}}^{\perp} \eta=0$.

Considere agora $U=\{p \in M \mid(\operatorname{grad} f)(p) \neq 0\}$. Mostraremos que $U=\emptyset$. Para isto, assumimos que $U \neq \emptyset$ e fazemos $e_{1}=\frac{\operatorname{grad} f}{\|\operatorname{grad} f\|}$. Como $e_{1}$ e $e_{2}$ são ortogonais, temos

$$
e_{2} f=0, \quad \operatorname{grad} f=\left(e_{1} f\right) e_{1} .
$$

Segue que

$$
\left\langle\mathrm{II}\left(e_{1}, e_{1}\right), \eta\right\rangle=\left\langle\mathrm{S}_{\eta}\left(e_{1}\right), e_{1}\right\rangle=\left\langle-f e_{1}, e_{1}\right\rangle=-f
$$

e

$$
\left\langle\mathrm{II}\left(e_{1}, e_{2}\right), \eta\right\rangle=\left\langle\mathrm{S}_{\eta}\left(e_{1}\right), e_{2}\right\rangle=\left\langle-f e_{1}, e_{2}\right\rangle=0 .
$$

Usando $2 \mathrm{H}=\mathrm{II}\left(e_{1}, e_{1}\right)+\mathrm{II}\left(e_{2}, e_{2}\right)$, temos que

$$
\mathrm{II}\left(e_{1}, e_{1}\right)=-f \eta, \quad \mathrm{II}\left(e_{1}, e_{2}\right)=0, \quad \mathrm{II}\left(e_{2}, e_{2}\right)=3 f \eta,
$$


e então

$$
\left\|\mathrm{S}_{\eta}\right\|^{2}=10 f^{2} .
$$

Denotaremos por $\left\{\omega_{1}, \omega_{2}\right\}$ as 1-formas duais a $\left\{e_{1}, e_{2}\right\}$ e por $\omega_{i}^{j}$ as 1-formas da conexão dadas por $\nabla_{(-)} e_{i}=\omega_{i}^{j}(-) e_{j}$, Vamos calcular $e_{2} f$ e $e_{1} f$. Como $\mathbb{S}^{3}$ possui curvatura seccional constante e $M$ é uma hipersuperfície, as equações de Codazzi nos dão

$$
\begin{aligned}
0 & =\left\langle\nabla_{e_{1}}^{\mathbb{S}^{3}} \mathrm{II}\left(e_{2}, e_{1}\right), \eta\right\rangle-\left\langle\mathrm{II}\left(\nabla_{e_{1}} e_{2}, e_{1}\right), \eta\right\rangle-\left\langle\mathrm{II}\left(e_{2}, \nabla_{e_{1}} e_{1}\right), \eta\right\rangle \\
& -\left\langle\nabla_{e_{2}}^{\mathbb{S}^{3}} \mathrm{II}\left(e_{1}, e_{1}\right), \eta\right\rangle+\left\langle\mathrm{II}\left(\nabla_{e_{2}} e_{1}, e_{1}\right), \eta\right\rangle+\left\langle\mathrm{II}\left(e_{1}, \nabla_{e_{2}} e_{1}\right), \eta\right\rangle \\
& =\omega_{2}^{1}\left(e_{1}\right) f-3 f \omega_{1}^{2}\left(e_{1}\right)+e_{2}(f)+f\left\langle\nabla_{e_{2}}^{\mathbb{S}^{3}} \eta, \eta\right\rangle-2 \omega_{1}^{1}\left(e_{2}\right) f
\end{aligned}
$$

e

$$
\begin{aligned}
0 & =\left\langle\nabla_{e_{1}}^{\mathbb{S}^{3}} \mathrm{II}\left(e_{2}, e_{2}\right), \eta\right\rangle-\left\langle\mathrm{II}\left(\nabla_{e_{1}} e_{2}, e_{2}\right), \eta\right\rangle-\left\langle\mathrm{II}\left(e_{2}, \nabla_{e_{1}} e_{2}\right), \eta\right\rangle \\
& -\left\langle\nabla_{e_{2}}^{\mathbb{S}^{3}} \operatorname{II}\left(e_{1}, e_{2}\right), \eta\right\rangle+\left\langle\mathrm{II}\left(\nabla_{e_{2}} e_{1}, e_{2}\right), \eta\right\rangle+\left\langle\mathrm{II}\left(e_{1}, \nabla_{e_{2}} e_{2}\right), \eta\right\rangle \\
& =3 e_{1}(f)+3 f\left\langle\nabla_{e_{1}}^{\mathbb{S}^{3}} \eta, \eta\right\rangle-6 f \omega_{2}^{2}\left(e_{1}\right)+3 f \omega_{1}^{2}\left(e_{2}\right)-f \omega_{2}^{1}\left(e_{2}\right) .
\end{aligned}
$$

Observando que $\omega_{j}^{i}=-\omega_{i}^{j}$, resulta

$$
e_{2} f=-4 f \omega_{2}^{1}\left(e_{1}\right), \quad 3 e_{1} f=-4 f \omega_{1}^{2}\left(e_{2}\right)
$$

Usando (5.7) e (5.12) vemos que $\omega_{2}^{1}\left(e_{1}\right)=0$ e, consequentemente, $d \omega_{1}=0$. Portanto (localmente) $\omega_{1}$ é exata, isto é, $\omega_{1}=d u$ para alguma função $u$. Como $d f=\left(e_{1} f\right) \omega_{1}+\left(e_{2} f\right) \omega_{2}$ e $e_{2} f=0$, temos que $d f \wedge \omega^{1}=0$, portanto, $f$ é uma função apenas de $u$. Denotando por $f^{\prime}$ e $f^{\prime \prime}$ a primeira e a segunda derivadas de $f$ com respeito a $u$, a segunda fórmula de (5.12) implica em

$$
4 f \omega_{1}^{2}=-3 f^{\prime} \omega^{2}
$$

Novamente, (5.7) e (5.12) nos dão

$$
\begin{aligned}
-4 f \triangle^{M} f & =\omega_{2}^{1}\left(e_{2}\right) e_{1}(f)-4 f\left(e_{1}\left(e_{1}(f)\right)\right. \\
& =3 f^{\prime 2}-4 f f^{\prime \prime}
\end{aligned}
$$

e, da primeira equação do sistema (5.6) e (5.9), obtemos

$$
4 f f^{\prime \prime}-3 f^{\prime 2}+8 f^{2}-40 f^{4}=0
$$

Pondo $\left(f^{\prime}\right)^{2}=y$ e usando que $f^{\prime} \neq 0$, de (5.15) segue que

$$
2 f \frac{d y}{d f}-3 y=40 f^{4}-8 f^{2}
$$

que implica (ver, por exemplo, [5])

$$
f^{\prime 2}=8 f^{4}-8 f^{2}+C f^{\frac{3}{2}}, \quad C \in \mathbb{R} .
$$


Por outro lado, a equação de Gauss nos dá que a curvatura Gaussiana é dada por:

$$
K=1+\operatorname{det} \mathrm{S}_{\eta}
$$

e

$$
\left\{\begin{array}{l}
K=1-3 f^{2} \\
d \omega_{1}^{2}=-K \omega^{1} \wedge \omega^{2} .
\end{array}\right.
$$

De (5.8), (5.13) e (5.18), obtemos

$$
4 f f^{\prime \prime}-7 f^{\prime 2}+16 f^{4}-\frac{16}{3} f^{2}=0
$$

logo (5.15) e (5.19), implicam em

$$
f^{\prime 2}=14 f^{4}-\frac{10}{3} f^{2}
$$

As condições (5.17) e (5.20) juntas dizem que $f$ deve satisfazer uma equação polinomial com coeficientes constantes, ou seja, $f$ é constante. Portanto, $M$ possui curvatura média constante e $\left\|\mathrm{S}_{\eta}\right\|^{2}=\|\mathrm{II}\|^{2}=2$.

A outra parte da equivalência segue imediatamente do sistema (5.4).

A seguir, vamos provar o teorema que classifica as superfícies $M^{2} \subset \mathbb{S}^{3}$ de curvatura média constante e $\|\mathrm{II}\|^{2}=2$. Faremos a demonstração de acordo com [8]. Ressaltamos que este teorema foi provado também por Z.H Hou em [25], com um diferente método.

Teorema 5.2.2. Seja $M$ uma hipersuperfície com curvatura média constante e $\|$ II $\|^{2}=2$.

(a) Se $M$ não é compacta, então é localmente parte de uma hiperesfera $\mathbb{S}^{2}\left(\frac{1}{\sqrt{2}}\right)$ ou de um toro de Clifford.

(b) Se $M$ é compacta e orientável, então $M$ é ou uma hiperesfera $\mathbb{S}^{2}\left(\frac{1}{\sqrt{2}}\right)$ ou um toro de Clifford.

Demonstração. Primeiro mostraremos que se $\|\mathrm{H}\|=$ constante e $\|\mathrm{II}\|^{2}=2$, então, os autovalores do operador de forma de $M \subset \mathbb{S}^{3}$ são ou $\lambda_{1}=\lambda_{2}= \pm 1$ ou $\lambda_{1}=-\lambda_{2}= \pm 1$.

Supomos $\|\mathrm{H}\|=$ constante $>0$. Seja $\eta=\frac{\mathrm{H}}{\|\mathrm{H}\|}$ uma seção unitária global no fibrado $T^{\perp} M$. Denotamos por $\lambda_{1}(p), \lambda_{2}(p)$ os autovalores de $\mathrm{S}_{\eta}$ em um ponto $p \in M$. De $\|\mathrm{H}\|=$ constante e $\|$ II $\|=$ constante resulta que $\lambda_{1}, \lambda_{2}$ são funções constantes sobre $M$, podemos supor que $\lambda_{1} \leq \lambda_{2}$. Nestas condições dois casos devem ser considerados. 
1. $\lambda_{1}=\lambda_{2}(= \pm 1)$.

Neste caso $M$ é umbílica em $\mathbb{S}^{3}$, logo é (uma parte de) $\mathbb{S}^{2}\left(\frac{1}{\sqrt{2}}\right)$.

2. $\lambda_{1} \neq \lambda_{2}$.

Seja $\left\{e_{1}, e_{2}\right\}$ uma base local ortonormal tal que

$$
\mathrm{S}_{\eta}\left(e_{1}\right)=\lambda_{1} e_{1} \quad \text { e } \quad \mathrm{S}_{\eta}\left(e_{2}\right)=\lambda_{2} e_{2}
$$

Consideramos $\omega_{1}, \omega_{2}$ as 1-formas duais à base $\left\{e_{1}, e_{2}\right\}$ e $\omega_{i}^{j}$ as 1-formas da conexão como no teorema anterior. Da equação de Codazzi (para o caso de formas espaciais) temos

$$
\nabla_{e_{1}} \mathrm{~S}_{\eta}\left(e_{2}\right)-\nabla_{e_{2}} \mathrm{~S}_{\eta}\left(e_{1}\right)=\mathrm{S}_{\eta}\left(\left[e_{1}, e_{2}\right]\right) .
$$

Como

$$
\left[e_{1}, e_{2}\right]=\nabla_{e_{1}} e_{2}-\nabla_{e_{2}} e_{1}=\omega_{2}^{1}\left(e_{1}\right) e_{1}-\omega_{1}^{2}\left(e_{2}\right) e_{2}
$$

obtemos

$$
\lambda_{2} \omega_{2}^{1}\left(e_{1}\right) e_{1}-\lambda_{1} \omega_{1}^{2}\left(e_{2}\right) e_{2}=\lambda_{1} \omega_{2}^{1}\left(e_{1}\right) e_{1}-\lambda_{2} \omega_{1}^{2}\left(e_{2}\right) e_{2}
$$

Utilizando a condição $\lambda_{1} \neq \lambda_{2}$, concluímos que $\omega_{1}^{2}=0$. Da segunda equação do sistema (5.18) segue que $K=0$ e, como $K=1+\lambda_{1} \lambda_{2}$, resulta que $\lambda_{1} \lambda_{2}=-1$. Usando $\|\mathrm{II}\|^{2}=2$, obtemos $\lambda_{1}=-1$ e $\lambda_{2}=1$ e, consequentemente, $\|\mathrm{H}\|=0$, contradizendo $\|\mathrm{H}\|=$ constante $>0$.

Supomos, agora, que $\|\mathrm{H}\|=0$. Seja $\eta$ uma seção local unitária do fibrado normal $M$ em $\mathbb{S}^{3}$. Desta vez, $\lambda_{1}$ e $\lambda_{2}$ são contínuas no domínio do sistema de coordenadas locais $U$. Como $\mathrm{H}=0$ e $\|\mathrm{II}\|^{2}=2$ temos que $\lambda_{1}=-1$ e $\lambda_{2}=1$. Se $M$ é orientável, então $\eta$ está definida em toda $M$, e o mesmo vale para $\lambda_{1}$ e $\lambda_{2}$. Utilizamos, então, o Teorema 4.8 de [17] para garantir que $M$ é o (uma parte do) toro de Clifford.

Segue da Observação 1.2.6 que o toro de Clifford $\mathbb{S}^{1}\left(\frac{1}{\sqrt{2}}\right) \times \mathbb{S}^{1}\left(\frac{1}{\sqrt{2}}\right)$ é uma superfície harmônica de $\mathbb{S}^{3}$, de forma que, devido aos Teoremas 5.2.1 e 5.2.2, podemos estabelecer o seguinte resultado:

Teorema 5.2.3. Seja $M$ uma superfície bi-harmônica própria de $\mathbb{S}^{3}$.

(a) Se $M$ não é compacta, então $M$ é localmente $\mathbb{S}^{2}\left(\frac{1}{\sqrt{2}}\right) \subset \mathbb{S}^{3}$. 
(b) Se $M$ é compacta e orientável, então $M=\mathbb{S}^{2}\left(\frac{1}{\sqrt{2}}\right)$.

Terminaremos este capítulo observando que no caso de dimensão maior, não se sabe se existem subvariedades bi-harmônicas próprias de $N^{n}(c), n>3, c \leq 0$. Contudo, foram provados os seguintes resultados:

1. Toda curva bi-harmônica de $\mathbb{R}^{n}$ é um segmento aberto de reta (ver [19]).

2. Seja $M^{3}$ uma hipersuperfície de $\mathbb{R}^{4}$. Então $M$ é bi-harmônica se e somente se é mínima $(\operatorname{ver}[24])$.

3. Seja $M$ uma subvariedade de $\mathbb{S}^{n}$. Então $M$ é bi-harmônica em $\mathbb{R}^{n+1}$ se e somente se é mínima em $\mathbb{R}^{n+1}$ (ver [16]). 


\section{Bibliografia}

[1] G.B. Airy. On the strains in the interior of beams. Philos. Trans. R. Soc. London Ser. A, 153 (1863), 49-79.

[2] M. Bekkar. Exemples de surfaces minimales dans l'espace de Heisenberg. Rem. Sem. Fac. Sci. Univ. Cagliari 61 (1991), no. 2, 123-130.

[3] L. Bianchi. Gruppi continui e finiti. Ed. Zanichelli, Bologna (1928).

[4] W. Boothby. An introduction to differentiable manifolds and Riemannian geometry. Academic Press (1986).

[5] W.E. Boyce, R C. DiPrima. Elementary differential equations. Jonh Wiley \& Sons, Inc. $(2000)$.

[6] R. Caddeo, A. Gray. Curve e Superfici, Vol. I, Ed. CUEC. Cagliari (1996).

[7] R. Caddeo, S. Montaldo, C. Oniciuc. Biharmonic submanifolds in spheres. Israel J. Math. 130 (2002), 109-123.

[8] R. Caddeo, S. Montaldo, C. Oniciuc. Biharmonic submanifolds of $\mathbb{S}^{3}$. Int. J. Math., 12 (2001), 867-876.

[9] R. Caddeo, S. Montaldo, C. Oniciuc, P. Piu. The classification of biharmonic curves of Cartan-Vranceanu 3-dimensional spaces. Modern Trends in Geometry and Topology (2005), $121-131$.

[10] R. Caddeo, S. Montaldo, C. Oniciuc, P. Piu. The Euler-Lagrange method for biharmonic curves. Mediterranean Journal of Mathematics (2006), 449-465. 
[11] R. Caddeo, S. Montaldo, P. Piu. Biharmonic curves on a surface. Rend. Mat. Appl. 21 (2001), 143-157.

[12] R. Caddeo, C. Oniciuc, P. Piu. Explicit formulas for non-geodesic biharmonic curves of the Heisenberg group. Rend. Sem. Mat. Univ. Politec. Torino 62 (2004), 265-277.

[13] R. Caddeo, L. Vanhecke. Does " $\Delta^{2} d^{2-n}=0$ on a Riemannian manifold" implies flatness? Period. Math. Hungar. 17 (1986), 109-117.

[14] É. Cartan. Leçons sur la géométrie des espaces de Riemann. Gauthier Villars, Paris (1946).

[15] B.Y. Chen, S. Ishikawa. Biharmonic pseudo-Riemannian submanifolds in pseudo-Euclidean spaces. Kyushu J.Math., 52 (1998), 167-185.

[16] B.Y. Chen. Some open problems and conjectures on submanifolds of finite type. Soochow J. Math., 17 (1986), 109-117.

[17] M. Dajczer. Submanifolds and Isometric Immersions. Mathematics Lecture Series 13, Publish or Perish (1990).

[18] C. Delaunay. Sur la surface de révolution dont la courbure moyenne est constante. J. Math. pures et appl. Sér.1 6 (1869), 309-320.

[19] I. Dimitric. Submanifolds of $\mathbb{E}^{m}$ with biharmonic mean curvature vector. Bull. Inst. Math. Acad. Sinica 20 (1992), 53-65.

[20] M.P. do Carmo, M. Dajczer. Helicoidal surfaces with constant mean curvature. Tôhoku Math. J. 34, (1982), 425-435.

[21] J. Eells, L. Lemaire. Selected topics in harmonic maps, CBMS, 50, Amer. Math. Soc, (1983).

[22] J. Eells, J.H. Sampson. Harmonic mappings of Riemannian manifolds. Amer. J. Math. 86 (1964).

[23] G. Fubini, Sugli spazi che ammettono un gruppo continuo di movimenti. Ann. di Matem. Tomo 8, serie III, (1903), 39-82.

[24] T. Hasanis, T. Vlachos. Hypersurfaces in $E^{4}$ with constante mean curvature vector field. Math. Nachr. 172 (1995), 145-169. 
[25] Z.H. Hou. Hypersurfaces in sphere with constante mean curvature. Proc. Amer. Math. Soc. 125 (1997), 109-160.

[26] G.Y. Jiang. 2-harmonic isometric immersions between Riemannian manifolds. Chinese Ann. Math. Ser. A 7 (1986), 130-144.

[27] G.Y. Jiang. 2-harmonic maps and their first and second variation formulas. Chinese Ann. Math. Ser. A 7 (1986), 389-402.

[28] D. Laugwitz. Diferential and Riemannian geometry. Academic Press, (1965).

[29] J.C. Maxwell. On reciprocal diagrams in space, and their relation to Airy's function of stress. Proc. London. Math. Soc. 2 (1868), 102-105.

[30] C. Mei. Curve bimoniche con Euler-Lagrange, Tesi di Laurea, Università di Cagliari, (2004).

[31] C. Oniciuc. Biharmonic maps between Riemannian manifolds. An. Stiint. Univ. Al.I. Cuza Iasi Mat. (N.S.), 48 (2002), 237-248.

[32] P. Piu. Sur certains types de distributions non-intégrables totalement géodésiques. Thèse de Doctorat, Université de Haute-Alsace, Mulhouse (1988).

[33] L. Sario, M. Nakai, C. Wang, L. Chung. Classification theory of Riemannian manifolds. Harmonic, quasiharmonic and biharmonic functions. Lecture Notes in Mathematics, Vol. 605. Springer-Verlag, Berlin-New York, 1977.

[34] H. Urakawa. Calculus of Variations and Harmonic Maps, Amer. Math. Soc, Providence, RI (1993).

[35] G. Vranceanu. Leçons de géométrie differentielle. Ed. Acad. Rep. Pop. Roum., vol. I, Bucarest (1957). 